McGill/99-16

hep-ph/9907281

\title{
Color Neutrality and the Gluon Distribution in a Very Large Nucleus
}

\author{
C.S. Lam ${ }^{*}$ and Gregory Mahlon ${ }^{\dagger}$ \\ Department of Physics, McGill University, \\ 3600 University St., Montréal, QC H3A 2T8 \\ Canada
}

(July 15, 1999)

\begin{abstract}
We improve the McLerran-Venugopalan model for the gluon distribution functions in very large nuclei by imposing the condition that the nucleons should be color neutral. We find that enforcing color neutrality cures the infrared divergences in the transverse coordinates which are present in the McLerran-Venugopalan model. Since we obtain well-defined expressions for the distribution functions, we are able to draw unambiguous conclusions about various features of the model. In particular, we show that the gluon distribution functions in the absence of quantum corrections behave as $1 / x_{F}$ to all orders in the coupling constant. Furthermore, our distribution functions exhibit saturation at small transverse momenta. The normalization of the distribution function we obtain is not arbitrary but specified in terms of the nucleon structure. We derive a sum rule for the integral of the gluon distribution function over transverse momenta, and show that the non-Abelian contributions serve only to modify the shape of the transverse momentum distribution. We obtain a relatively simple expression for the mean value of the transverse momentum-squared. The connection between the McLerranVenugopalan model and the Dokshitzer-Gribov-Lipatov-Altarelli-Parisi equation is discussed quantitatively. Finally, we illustrate our results in terms of a simple nuclear model due to Kovchegov.
\end{abstract}

24.85.+p, 12.38.Bx

Typeset using REVTEX 


\section{INTRODUCTION}

Several years ago, McLerran and Venugopalan [1] recognized the existence of a regime in which the gluon structure functions ought to be calculable within quantum chromodynamics (QCD). Their idea is to recognize that in large nuclei at small enough values of the longitudinal momentum fraction $x_{F}$, the density of partons per unit area is large. Since a large number of charges are contributing, it is expected that classical methods should apply, i.e. the vector potential is computed from the classical Yang-Mills equations, and quantum correlation functions are approximated by ensemble averages. This picture was developed more fully in subsequent work [2 4], and is somewhat related to the approach of Mueller [5 10].

In the region of very small $x_{F}$, the quantum corrections to the distribution functions calculated in the McLerran-Venugopalan model become large [4, 11]. In particular, these corrections are enhanced by powers of $\alpha_{s} \ln \left(1 / x_{F}\right)$, implying that the classical approximation being made at lowest order is breaking down in this region. This observation has lead to the development of a renormalization group procedure [12] whereby these corrections are taken into account by incorporating gluons with large values of $x_{F}$ into the charge density. This approach, which uses the results of the McLerran-Venugopalan model as its input, has been subsequently developed in Refs. [12 18.

The focus of this paper is not on the very small $x_{F}$ region, but rather on dealing with the infrared divergence in the transverse coordinates which appears in the McLerranVenugopalan model. The vector potential two-point correlation functions obtained in Ref. [12 are highly infrared divergent, going like $\left(x^{2}\right)^{x^{2}}$ at large distances. On one hand, the region where this poor behavior manifests itself is confined to separations $x \gg \Lambda_{\mathrm{QCD}}^{-1}$. This is firmly in the non-perturbative regime where one does not really believe the results of a perturbative calculation anyway. However, since the gluon number density is derived from the Fourier transform of this correlation function, the presence of such a strong divergence makes it difficult to obtain more than qualitative results from the theory: one must resort to cutting off the transverse spatial integrations at $\Lambda_{\mathrm{QCD}}^{-1}$ [19.

In this paper we will demonstrate that the infrared divergence described above is closely related to the issue of color neutrality. Physically, nucleons appear to be colorless when viewed at length scales which greatly exceed $\Lambda_{\mathrm{QCD}}^{-1}$. Consequently, we expect that twopoint correlation functions should die off rapidly beyond distance scales of about $\Lambda_{\mathrm{QCD}}^{-1}$, because of confinement. The quarks in two widely separated nucleons should not feel each others presence. Thus, the fundamental requirement that the nucleons be color neutral may be rephrased as a restriction on the allowable charge-density correlation functions $\mathcal{D}(\boldsymbol{x})$. Although the McLerran-Venugopalan model employs an ensemble of charge distributions which respects the fact that the average charge should vanish, the two-point correlator computed from this ensemble is inconsistent with color-neutral nucleons. We claim that the color neutrality condition should be viewed as of primary importance, and that we should modify the ensemble of charge distributions to satisfy it. By doing so, we incorporate one of the major effects brought about by confinement, parameterizing it in terms of the precise form chosen for $\mathcal{D}(\boldsymbol{x})$. Enforcing color neutrality cures the infrared divergence in the McLerran-Venugopalan model by introducing a new scale into the problem, namely the minimum length scale for which color neutrality holds. This outcome is somewhat similar to the one considered in Refs. [20,21]; however, neither of these papers examine the 
consequences of enforcing color neutrality in great detail.

Given a model which incorporates the color neutrality of the nucleons at some scale, we obtain infrared finite correlation functions. Because our results are well-defined everywhere, we are able to draw firm conclusions about various properties of the gluon distribution functions, in spite of their highly nonlinear form. We are able to demonstrate that the gluon distribution functions in the McLerran-Venugopalan model are proportional to $1 / x_{F}$, independent of the distribution of charge in the longitudinal coordinate. To evade this conclusion apparently requires charge-density correlation functions which have a non-trivial longitudinal structure. Although we have modified the form of $\mathcal{D}(\boldsymbol{x})$ from the one employed in Ref. [12, we still obtain correlation functions which are consistent with asymptotic freedom. That is, in the ultraviolet limit, the non-Abelian terms drop out and we obtain a gluon distribution function which goes like $1 / \boldsymbol{q}^{2}$. At small values of the transverse momentum $\boldsymbol{q}$, our gluon distribution functions either approach a finite constant or diverge mildly (like $\ln \boldsymbol{q}^{2}$ ), depending on the long distance behavior of $\mathcal{D}(\boldsymbol{x})$. The density of gluons at small $\boldsymbol{q}$ grows more slowly with increasing nuclear size than the number of nucleons, consistent with saturation [10,22 24]. We also derive a transverse momentum sum rule, which shows that the non-Abelian corrections only serve to transfer gluons (at fixed $x_{F}$ ) from one value of transverse momentum to another: the total number of gluons is not altered. This is reflected in the fact that the structure functions we obtain are simply proportional to the number of nucleons: all non-linear dependence on the amount of charge present is suppressed at large values of the momentum transfer. As an application of the sum rule, we make contact with the Dokshitzer-Gribov-Lipatov-Altarelli-Parisi equation [25,26], demonstrating the plausibility of the McLerran-Venugopalan model. In addition, we are able to compute a fairly simple expression for the mean transverse momentum-squared of the gluons which is accurate to order unity: that is, the logarithms that arise in our calculation are all evaluated at a scale which is completely calculable given a nucleon model. We find that the leading $Q^{2}$ behavior of this quantity is linear in the number of nucleons, whereas the logarithmic corrections are quadratic in the charge per unit transverse area.

The remainder of this paper is organized as follows. In Sec. [1], we review the McLerranVenugopalan model as described in Refs. [1, 1, 12]. We allow for a generalized dependence of the charge-density correlator on the transverse coordinates in order to set the stage for the subsequent discussion. In Sec. III] we describe the conditions which the charge-density correlator must satisfy in order to be consistent with nucleons which are color neutral. We examine the properties of the resulting gluon number density in Sec. $\mathbb{I V}$. If the chargedensity correlator is Gaussian and local in the longitudinal coordinate, we show that the gluon number density is always proportional to $1 / x_{F}$. We derive a sum rule for the integral of the gluon number density over the transverse momenta, and apply it to the computation of the gluon distribution function at large values of the momentum transfer. Our result is closely related to the Dokshitzer-Gribov-Lipatov-Altarelli-Parisi equation. Sec. IV concludes with a discussion of the mean value of the transverse momentum-squared obtained by our approach. We illustrate our results in Sec. $\square$ within the context of a specific nuclear model by Kovchegov [27]. Finally, Sec. VI contains our conclusions. A discussion of our notation and conventions may be found in Appendix A. The details of how to evaluate various integrals arising in the text are contained in Appendices $\mathrm{B}, \mathrm{Q}$, and $\mathrm{D}$. 


\section{REVIEW OF THE MCLERRAN-VENUGOPALAN MODEL}

\section{A. General Considerations}

The goal of the McLerran-Venugopalan (MV) model is to compute the gluon distribution function at small values of the longitudinal momentum fraction $x_{F} \equiv q_{\text {gluon }}^{+} / Q_{\text {nucleon }}^{+}$ for a very large nucleus. We begin our discussion with the assumptions which form the basis of the MV model, and outline the steps in the computation of the gluon distribution function. Having described the ingredients which underlie the calculations, we will explain the conditions which are required for this picture to be applicable.

Consider a nucleus moving along the $z$ axis at nearly the speed of light. The central assertion of the MV model is that, in a specific kinematic regime, we may determine the gluon distribution function of the nucleus as follows. First, we are told to compute the classical vector potential generated by the valence quarks. Since the valence quarks appear as a Lorentz-contracted pancake-shaped distribution of color charge, the current appearing in the classical Yang-Mills equations takes the form (see Appendix A for a summary of our notation and conventions)

$$
\begin{aligned}
J^{+}(x) & =\rho\left(x^{-}, \boldsymbol{x}\right), \\
J^{-}(x) & =0, \\
\boldsymbol{J}(x) & =\mathbf{0} .
\end{aligned}
$$

There is no $x^{+}$dependence in Eq. (2.1) in the limit that the source (i.e. the nucleus) is moving at the speed of light. Because the intuitive parton model is realized in the light-cone gauge 28 31, we should write the solution in that gauge, i.e. $A^{+} \equiv 0$.

By definition, the gluon number density is just the gluon number operator evaluated in the (quantum) state of interest. This may be expressed in terms of the two-point vector potential correlation function as 23,29]

$$
\frac{d N}{d q^{+} d^{2} \boldsymbol{q}}=\frac{q^{+}}{4 \pi^{3}} \int_{-\infty}^{\infty} d x^{-} \int d^{2} \boldsymbol{x} \int_{-\infty}^{\infty} d x^{-} \int d^{2} \boldsymbol{x}^{\prime} e^{-i q^{+}\left(x^{-}-x^{-}\right)} e^{i \boldsymbol{q} \cdot\left(\boldsymbol{x}-\boldsymbol{x}^{\prime}\right)}\left\langle A_{i}^{a}\left(x^{-}, \boldsymbol{x}\right) A_{i}^{a}\left(x^{\prime-}, \boldsymbol{x}^{\prime}\right)\right\rangle .
$$

In the MV model, the average over quantum fluctuations implied by the angled brackets on the right hand side of (2.2) is replaced by a classical average over a suitably weighted ensemble of charge densities. The ensemble should consist of all of the physically realizable states of the quarks (in color singlet combinations) within the nucleons and of the nucleons within the nucleus. Then, what we require is an expression for the vector potential in terms of the charge density plus the probability distribution for determining how likely a given charge configuation is. In the MV model, ensemble averages are computed according to the Gaussian weight

$$
\int[d \rho] \exp \left\{-\int d x^{-} d^{2} \boldsymbol{x} \int d x^{\prime-} d^{2} \boldsymbol{x}^{\prime} \operatorname{Tr}\left[\rho\left(x^{-}, \boldsymbol{x}\right) \rho\left(x^{\prime-}, \boldsymbol{x}^{\prime}\right)\right] \frac{\delta\left(x^{-}-x^{--}\right)}{\mu^{2}\left(x^{-}\right)} \mathcal{D}^{-1}\left(\boldsymbol{x}-\boldsymbol{x}^{\prime}\right)\right\} .
$$

This weight implies that the charge density has an average value of zero. There are, however, fluctuations about this average value, the size of which are governed by $\mu^{2}\left(x^{-}\right)$, which is 
related to the average charge density squared per unit area per unit thickness. The precise statement of the relationship appears in Sec. [II]. The longitudinal dependence of Eq. (2.3) is purely local in $x^{-}$, reflecting the fact that the large number of quarks which contribute to the charge per unit area at a given transverse position typically come from different nucleons: hence, they should be uncorrelated. The transverse space dependence of the MV model as described in Refs. [1, [1, 12] is

$$
\mathcal{D}\left(\boldsymbol{x}-\boldsymbol{x}^{\prime}\right)=\delta^{2}\left(\boldsymbol{x}-\boldsymbol{x}^{\prime}\right) .
$$

Ultimately, we intend to modify this dependence. Therefore, we will write our expressions in terms of $\mathcal{D}$ and insert (2.4) only when we wish to specifically discuss the results of Ref. [12].

Thus, given the solution for the vector potential in terms of the charge density and an ansatz for the charge density correlation function, we may compute an approximation to the gluon distribution function. We now describe the conditions necessary for this procedure to yield a good approximation to the result which would be obtained in a full QCD treatment.

First, we note that the charge distribution for the nucleus is taken to be a Lorentzcontracted pancake moving along the $z$ axis at (nearly) the speed of light. We wish to be in the regime where the partons do not resolve its longitudinal structure. This is only true for partons with very small longitudinal momentum fractions. To determine how small the momentum should be, we note that the corresponding length scale $x^{-} \sim 1 / q_{\text {gluon }}^{+}$should be larger than the thickness of the Lorentz-contracted nucleus. This thickness is of order $A^{1 / 3} a / \gamma$, where $A$ is the number of nucleons, $a$ the radius of a single nucleon, and $\gamma$ is the (common) boost factor of the nucleons which comprise the nucleus. Writing the mass of the nucleon to be $m$, we see then that the condition on the momentum fraction reads

$$
x_{F} \equiv \frac{q_{\text {gluon }}^{+}}{Q_{\text {nucleon }}^{+}} \lesssim \frac{A^{-1 / 3}}{m a} .
$$

Gluons with longitudinal momenta satisfying (2.5) cannot resolve distances shorter than the thickness of the Lorentz-contracted nucleus they see.

An immediate consequence of being unable to resolve nucleons with different values of $x^{-}$is that we may view the nucleons as being piled up onto effectively the same value of $x^{-}$. Thus, each unit of transverse area will, on average, contain a large number of valence quarks. Since there are a large number of quarks contributing, the total contribution will be in a large representation of the gauge group, allowing us to treat the source in the the classical approximation. The large number of quarks also allows us to justify the use of the Gaussian weight (2.3) via the central limit theorem. The number of valence quarks per unit area in a flattened nucleus of radius $R$ is $3 A / \pi R^{2} \sim 3 A^{1 / 3} / \pi a^{2}$, since the nuclear radius scales with the number of nucleons as $R \sim A^{1 / 3} a$. Gluons with a transverse momentum $\boldsymbol{q}^{2}$

\footnotetext{
${ }^{1}$ The authors of Ref. [12] choose to replace the spatial variable $x^{-}$with what they call the spacetime rapidity $y$, defined by $y \equiv y_{0}+\ln \left(x_{0}^{-} / x^{-}\right)$. Thus, they write $\mu^{2}(y)$ and refer to it as the average charge density squared per unit area per unit rapidity. We will stick to the description in terms of $x^{-}$in this paper.
} 
see a transverse area of about $\pi / \boldsymbol{q}^{2}$. Thus, to maintain the condition that there is a large number of quarks in the region being probed, we should only look at transverse momenta such that

$$
\Lambda_{\mathrm{QCD}}^{2} \lesssim \boldsymbol{q}^{2} \lesssim 3 A^{1 / 3} a^{-2}
$$

that is, $\boldsymbol{q}$ should be soft enough to correspond to a large enough area, but not so soft that the coupling becomes strong. A second factor limiting the maximum $\boldsymbol{q}^{2}$ for which this treatment is valid is the observation that if the transverse momentum of the emitted gluons is too large, then the eikonal approximation we are making breaks down. That is, the norecoil approximation that allowed us to treat the valence quarks as being localized on the light-cone fails.

The bottom line is that in large enough nuclei for small enough values of the longitudinal momentum carried by the parton and moderately large tranverse momenta, the number of quarks per unit area becomes large. In this regime, we expect that we may compute the gluon distribution function at lowest order from the classical solution to the Yang-Mills equations.

\section{B. Non-Abelian Weisäcker-Williams Fields}

As outlined above, the MV model makes use of the classical solution to the Yang-Mills equations in the presence of a source of the form (2.1), with $J^{+}(x) \equiv \rho\left(x^{-}, \boldsymbol{x}\right)$ the only nonzero component, independent of the light-cone time $x^{+}$. Ultimately, we will take the dependence on the light-cone distance $x^{-}$to be a delta function, corresponding to the pancakeshaped distribution described above. Initially, however, we will work with a finite spread in $x^{-}$(a multi-layer pancake), to avoid the ambiguities that would otherwise arise in the commutator terms of the Yang-Mills equations [12]. Furthermore, certain quantum corrections may be included by a renormalization group treatment 12 18] which generates a specific $x^{-}$ dependence, reflecting the presence of the Yukawa cloud of quark pairs and gluons around the original source. So, it behooves us to make provision for such a dependence.

The simplest route to the solution for the vector potential in the light-cone gauge for a current of the form (2.1) is to begin by finding the solution in the covariant gauge, and subsequently transform into the light-cone gauge [5.27]. Therefore, we begin in the covariant gauge, $\partial_{\mu} \widetilde{A}^{\mu}(x)=0$, where the field equations (A3) become

$$
\partial^{2} \widetilde{A}^{\nu}-2 i\left[\widetilde{A}^{\mu}, \partial_{\mu} \widetilde{A}^{\nu}\right]+i\left[\widetilde{A}_{\mu}, \partial^{\nu} \widetilde{A}^{\mu}\right]-\left[\widetilde{A}_{\mu},\left[\widetilde{A}^{\mu}, \widetilde{A}^{\nu}\right]\right]=J^{\nu}
$$

We recall that in electrodynamics, the $\mu$ th component of the vector potential is generated by the $\mu$ th component of the current (in the covariant gauge). Analogously, we attempt to form a solution where the only non-zero component is $\widetilde{A}^{+}$. Imposing this condition, we find that this is possible, provided that

$$
\partial^{2} \widetilde{A}^{+}-2 i\left[\widetilde{A}^{+}, \partial_{+} \widetilde{A}^{+}\right]=\rho .
$$

Since $\widetilde{A}^{-}$and $\widetilde{A}^{i}$ vanish, the condition that we are in covariant gauge reduces to $\partial_{+} \widetilde{A}^{+}=0$. Hence $\widetilde{A}^{+}$is independent of $x^{+}$and Eq. (2.8) reduces to 


$$
\boldsymbol{\nabla}^{2} \widetilde{A}^{+}\left(x^{-}, \boldsymbol{x}\right)=\rho\left(x^{-}, \boldsymbol{x}\right),
$$

Notice that Eq. (2.9) is local in $x^{-}$. This is a consequence of the $x^{+}$-independence of the vector potential, which reduces $\partial^{2}=-2 \partial^{+} \partial^{-}+\nabla^{2}$ to just $\nabla^{2}$. The solution of (2.9) is

$$
\widetilde{A}^{+}\left(x^{-}, \boldsymbol{x}\right)=\int d^{2} \boldsymbol{x}^{\prime} G\left(\boldsymbol{x}-\boldsymbol{x}^{\prime}\right) \rho\left(x^{-}, \boldsymbol{x}^{\prime}\right),
$$

where

$$
G\left(\boldsymbol{x}-\boldsymbol{x}^{\prime}\right) \equiv \frac{1}{4 \pi} \ln \left(\frac{\left|\boldsymbol{x}-\boldsymbol{x}^{\prime}\right|^{2}}{\lambda^{2}}\right)
$$

The arbitrary length scale $\lambda$ which appears in (2.11) reflects the lack of a scale in the classical theory. This same lack of scale leads to the infrared divergence in perturbation theory. Note that in the covariant gauge, the fact that $J$ and $A$ are color matrices has introduced no real complications into the solution: the vector potential is still linear in the sources.

The non-Abelian features make themselves felt when we gauge transform our solution to the light-cone gauge. We write the gauge transformation as

$$
A^{\mu}(x)=\mathrm{U}(x) \widetilde{A}^{\mu}(x) \mathrm{U}^{-1}(x)-i\left[\partial^{\mu} \mathrm{U}(x)\right] \mathrm{U}^{-1}(x) .
$$

Asking that the new gauge be the light-cone gauge, $A^{+} \equiv 0$, we find that $\mathrm{U}(x)$ should satisfy

$$
i\left[\partial_{-} \mathrm{U}(x)\right]=\mathrm{U}(x) \widetilde{A}^{+}(x) .
$$

The solution to Eq. 2.13) is the path-ordered exponential

$$
\mathrm{U}(x) \equiv \overline{\mathcal{P}} \exp \left[i \int_{-\infty}^{x^{-}} d \xi^{-} \widetilde{A}^{+}\left(\xi^{-}, \boldsymbol{x}\right)\right]
$$

where the sense of the path-ordering $\overline{\mathcal{P}}$ is such that

$$
\mathrm{U}(x) \equiv \mathbf{1}+\sum_{j=1}^{\infty} i^{j} \int_{-\infty}^{x^{-}} d \xi_{1}^{-} \int_{-\infty}^{\xi_{1}^{-}} d \xi_{2}^{-} \cdots \int_{-\infty}^{\xi_{j-1}^{-}} d \xi_{j}^{-} \widetilde{A}^{+}\left(\xi_{j}^{-}, \boldsymbol{x}\right) \cdots \widetilde{A}^{+}\left(\xi_{2}^{-}, \boldsymbol{x}\right) \widetilde{A}^{+}\left(\xi_{1}^{-}, \boldsymbol{x}\right)
$$

i.e. the factors are arranged such that $x^{-}$increases as the factors of $\widetilde{A}^{+}$are read from left to right. Using (2.12) and (2.14) to obtain the light-cone gauge expressions yields

$$
A^{-}(x)=0
$$

and

$$
\begin{aligned}
A^{j}(x) & =-i\left[\partial^{j} \mathrm{U}(x)\right] \mathrm{U}^{-1}(x) \\
& =\int_{-\infty}^{x^{-}} d \xi^{-} \mathrm{U}\left(\xi^{-}, \boldsymbol{x}\right)\left[\partial^{j} \widetilde{A}^{+}\left(\xi^{-}, \boldsymbol{x}\right)\right] \mathrm{U}^{-1}\left(\xi^{-}, \boldsymbol{x}\right) .
\end{aligned}
$$

The Abelian limit may be obtained from Eq. (2.17) by allowing all quantities to commute. Equivalently, we may take only those contributions which are linear in the source. 
It is instructive to recall the features of the solution in electrodynamics for a point charge moving along the $x^{3}$ axis at the speed of light. In this situation, Eq. (2.17) reduces to

$$
A^{j}(x)=\frac{e}{2 \pi} \Theta\left(x^{-}\right) \frac{x^{j}}{\boldsymbol{x}^{2}} .
$$

The only non-vanishing components of the field tensor produced by the vector potential in $(2.18)$ are

$$
F^{+j}(x)=-\frac{e}{2 \pi} \delta\left(x^{-}\right) \frac{x^{j}}{x^{2}} .
$$

These components of the field tensor correspond to the transverse electric and magnetic fields: the fact that $F^{-j}=0$ indicates that these fields have equal strength. There are no longitudinal fields generated in this limit. An observer sitting at some fixed position $\left(\boldsymbol{b}, x^{3}\right)$ would see no fields except at the instant when the charge made its closest approach. At this time a $\delta$-function pulse of transverse and mutually perpendicular electric and magnetic fields would be seen. The magnitude of the pulse would be proportional to $1 / b$. The (unobservable) vector potential, which was zero before the passage of the charge, takes on a non-zero value for all times afterward. Since the fields vanish at these times, the late-time vector potential may be thought of as some particular gauge transformation of the vacuum.

The overall features of the above description continue to hold when we switch to QCD, although the details are slightly altered by the presence of the path-ordered exponentials, which introduce a nonlinear dependence on the source. The net effect of these factors is to color-rotate the source in a complicated fashion. Nevertheless, the chromoelectric and chromomagnetic fields are non-zero only at the instant of closest approach by the charge, and the vector potential switches from one gauge transform of the vacuum to a different one at this instant.

\section{Structure of the Correlation Function}

Having obtained the solution for $\boldsymbol{A}$ in terms of $\rho$ via Eqs. (2.10), (2.14) and (2.17), we now turn to the question of actually performing the color averaging. The Gaussian weight (2.3) argued for above leads to the two-point charge density correlator

$$
\left\langle\rho^{a}\left(x^{-}, \boldsymbol{x}\right) \rho^{b}\left(x^{\prime-}, \boldsymbol{x}^{\prime}\right)\right\rangle=\delta^{a b} \mu^{2}\left(x^{-}\right) \delta\left(x^{-}-x^{\prime-}\right) \mathcal{D}\left(\boldsymbol{x}-\boldsymbol{x}^{\prime}\right) .
$$

Thus, to compute the correlation function $\left\langle A_{i}^{a}\left(x^{-}, \boldsymbol{x}\right) A_{j}^{b}\left(x^{\prime-}, \boldsymbol{x}^{\prime}\right)\right\rangle$, we expand $\boldsymbol{A}$ in terms of $\rho$ with the help of Eqs. (2.10), (2.14), and (2.17), and perform all possible contractions on pairs of $\rho$ 's using (2.20). At the end of this process, we resum the series. The details of this calculation may be found in Ref. [12]. Here, we shall simply quote the result:

$$
\left\langle A_{i}^{a}\left(x^{-}, \boldsymbol{x}\right) A_{j}^{b}\left(x^{\prime-}, \boldsymbol{x}^{\prime}\right)\right\rangle=\frac{\delta^{a b}}{N_{c}} \frac{\partial_{i} \partial_{j}^{\prime} L\left(\boldsymbol{x}-\boldsymbol{x}^{\prime}\right)}{L\left(\boldsymbol{x}-\boldsymbol{x}^{\prime}\right)}\left\{\exp \left[N_{c} \chi\left(x^{-}, x^{\prime-}\right) L\left(\boldsymbol{x}-\boldsymbol{x}^{\prime}\right)\right]-1\right\} .
$$

Eq. (2.21) depends on two new functions. The first, $\chi\left(x^{-}, x^{\prime-}\right)$, is the total charge-squared per unit area at positions to the left of the leftmost of $\left(x^{-}, x^{\prime-}\right)$ : 


$$
\chi\left(x^{-}, x^{\prime-}\right) \equiv \int_{-\infty}^{\min \left(x^{-}, x^{-}\right)} d \xi^{-} \mu^{2}\left(\xi^{-}\right) .
$$

The appearance of such an expression is not surprising when we recall that the value of the vector potential depends on whether or not the charge has yet reached its point of closest approach. What $\chi\left(x^{-}, x^{-}\right)$measures is the amount of charge in those layers of the source which have already passed both of the points which we are comparing. Although the range of integration extends to $x^{-}=-\infty$, in practice this is cut off by the form of $\mu^{2}\left(x^{-}\right)$, which for a pancake-shaped charge distribution, should be non-zero only in a relatively small range around the position of the nucleus along the $x^{-}$axis.

The second new function appearing in (2.21) is given by

$$
\begin{array}{r}
L\left(\boldsymbol{x}-\boldsymbol{x}^{\prime}\right) \equiv \int d^{2} \boldsymbol{\xi} \int d^{2} \boldsymbol{\xi}^{\prime} \mathcal{D}\left(\boldsymbol{\xi}-\boldsymbol{\xi}^{\prime}\right)\left[G(\boldsymbol{x}-\boldsymbol{\xi}) G\left(\boldsymbol{x}^{\prime}-\boldsymbol{\xi}^{\prime}\right)-\frac{1}{2} G(\boldsymbol{x}-\boldsymbol{\xi}) G\left(\boldsymbol{x}-\boldsymbol{\xi}^{\prime}\right)\right. \\
\left.-\frac{1}{2} G\left(\boldsymbol{x}^{\prime}-\boldsymbol{\xi}\right) G\left(\boldsymbol{x}^{\prime}-\boldsymbol{\xi}^{\prime}\right)\right]
\end{array}
$$

Note that this function vanishes when $\boldsymbol{x}=\boldsymbol{x}^{\prime}$. Thus, at very short distances the nonlinear terms in (2.21) drop out and the behavior of the correlation function is the same as in the Abelian case:

$$
\lim _{\left|\boldsymbol{x}-\boldsymbol{x}^{\prime}\right| \rightarrow 0}\left\langle A_{i}^{a}\left(x^{-}, \boldsymbol{x}\right) A_{j}^{b}\left(x^{\prime-}, \boldsymbol{x}^{\prime}\right)\right\rangle=\delta^{a b} \chi\left(x^{-}, x^{\prime-}\right)\left[\partial_{i} \partial_{j}^{\prime} L\left(\boldsymbol{x}-\boldsymbol{x}^{\prime}\right)\right] .
$$

In this limit, all of the dependence on the transverse coordinates is contained in the derivative of $L$ :

$$
\begin{aligned}
\partial_{i} \partial_{j}^{\prime} L\left(\boldsymbol{x}-\boldsymbol{x}^{\prime}\right) & =\int d^{2} \boldsymbol{\xi} \int d^{2} \boldsymbol{\xi}^{\prime} \mathcal{D}\left(\boldsymbol{\xi}-\boldsymbol{\xi}^{\prime}\right)\left[\partial_{i} G(\boldsymbol{x}-\boldsymbol{\xi})\right]\left[\partial_{j} G\left(\boldsymbol{x}^{\prime}-\boldsymbol{\xi}^{\prime}\right)\right] \\
& \equiv \delta_{i j} \mathcal{L}\left(\boldsymbol{x}-\boldsymbol{x}^{\prime}\right)+\mathcal{L}_{i j}\left(\boldsymbol{x}-\boldsymbol{x}^{\prime}\right)
\end{aligned}
$$

To uniquely specify the decomposition appearing on the second line of (2.25) we take $\mathcal{L}_{i j}$ to be traceless. Hence, the only piece of (2.25) which contributes to the gluon number density is $\delta_{i j} \mathcal{L}\left(\boldsymbol{x}-\boldsymbol{x}^{\prime}\right)$.

\section{Result Assuming Purely Local Charge Density Correlations}

As mentioned earlier, the results presented in Ref. [12] utilize a purely local correlator for the charge density correlation function, i.e. Eq. (2.4). Inserting such a $\delta$-function dependence into (2.23) produces

$$
L\left(\boldsymbol{x}-\boldsymbol{x}^{\prime}\right)=\gamma\left(\boldsymbol{x}-\boldsymbol{x}^{\prime}\right)-\gamma(\mathbf{0})
$$

where, formally,?

\footnotetext{
${ }^{2}$ The authors of Ref. [12] write $\gamma(\boldsymbol{x})=\frac{1}{\nabla^{4}} \delta^{2}(\boldsymbol{x})$, whereas we obtain the convolution of $\frac{1}{\nabla^{2}} \delta^{2}(\boldsymbol{x})$ with itself. To demonstrate the equivalence of these two quantities requires that we employ a consistent regularization scheme for the infrared divergences, for example by doing the entire computation in $2+\varepsilon$ dimensions.
} 


$$
\gamma\left(\boldsymbol{x}-\boldsymbol{x}^{\prime}\right)=\int d^{2} \boldsymbol{\xi} G(\boldsymbol{x}-\boldsymbol{\xi}) G\left(\boldsymbol{x}^{\prime}-\boldsymbol{\xi}\right)
$$

This function contains a quadratic infrared divergence, on top of the arbitrary length scale introduced by the logarithmic divergence of $G\left(\boldsymbol{x}-\boldsymbol{x}^{\prime}\right)$ itself. Fortunately, it is only the combination $\gamma\left(\boldsymbol{x}-\boldsymbol{x}^{\prime}\right)-\gamma(\mathbf{0})$ which appears in the correlation function. Thus, the quadratic part of the divergence drops out. There is still a logarithmic dependence on the infrared cutoff. The authors of Ref. [12] take

$$
\gamma\left(\boldsymbol{x}-\boldsymbol{x}^{\prime}\right)-\gamma(\mathbf{0})=\frac{1}{16 \pi}\left|\boldsymbol{x}-\boldsymbol{x}^{\prime}\right|^{2} \ln \left(\left|\boldsymbol{x}-\boldsymbol{x}^{\prime}\right|^{2} \Lambda_{\mathrm{QCD}}^{2}\right)
$$

where they have chosen on physical grounds to set this cutoff to $\Lambda_{\mathrm{QCD}}^{-1}$. The derivative of $(2.28)$ is

$$
\begin{aligned}
\partial_{i} \partial_{j}^{\prime} \gamma\left(\boldsymbol{x}-\boldsymbol{x}^{\prime}\right)= & -\frac{\delta_{i j}}{8 \pi}\left[\ln \left(\left|\boldsymbol{x}-\boldsymbol{x}^{\prime}\right|^{2} \Lambda_{\mathrm{QCD}}^{2}\right)+2\right] \\
& +\frac{1}{4 \pi}\left[\frac{1}{2} \delta_{i j}-\frac{\left(x-x^{\prime}\right)_{i}\left(x-x^{\prime}\right)_{j}}{\left|\boldsymbol{x}-\boldsymbol{x}^{\prime}\right|^{2}}\right]
\end{aligned}
$$

Note that the terms on the second line of $(2.29)$ are traceless, and do not contribute to the gluon number density (2.2).

Because $\gamma\left(\boldsymbol{x}-\boldsymbol{x}^{\prime}\right)$ is formally the inverse of $\boldsymbol{\nabla}^{4}$, when we form the trace $\partial_{i} \partial_{i}^{\prime} \gamma\left(\boldsymbol{x}-\boldsymbol{x}^{\prime}\right)$ that appears in (2.21), we should obtain the inverse of $\nabla^{2}$ [i.e. Eq. (2.11)]. Eq. (2.29) satisfies this expectation, since the extra " +2 " appearing in the first line may be absorbed into the arbitrary scale. What this means is that in a strict evaluation of Eq. (2.21), if we want the numerator factor $\partial_{i} \partial_{j}^{\prime} \gamma\left(\boldsymbol{x}-\boldsymbol{x}^{\prime}\right)$ to consist of a single logarithmic term, then that logarithm must contain a different scale from the one used in $L\left(\boldsymbol{x}-\boldsymbol{x}^{\prime}\right)$ itself. On the other hand, in the qualitative treatment of the infrared employed in Ref. [12], both logarithms are assumed to have the same scale $\left(\sim \Lambda_{\mathrm{QCD}}\right)$, producing

$$
\left\langle A_{i}^{a}\left(x^{-}, \boldsymbol{x}\right) A_{i}^{a}\left(x^{\prime-}, \boldsymbol{x}^{\prime}\right)\right\rangle=\frac{4\left(N_{c}^{2}-1\right)}{N_{c}\left|\boldsymbol{x}-\boldsymbol{x}^{\prime}\right|^{2}}\left[1-\left(\left|\boldsymbol{x}-\boldsymbol{x}^{\prime}\right|^{2} \Lambda_{\mathrm{QCD}}^{2}\right)^{\left(N_{c} / 16 \pi\right) \chi\left(x^{-}, x^{-}\right)\left|\boldsymbol{x}-\boldsymbol{x}^{\prime}\right|^{2}}\right] .
$$

According to Eq. (2.2), the gluon number density depends on the Fourier transform of (2.30). Unfortunately, there is a problem with the correlation function appearing in Eq. (2.30): it diverges like $\left(x^{2}\right)^{x^{2}}$ at large distances. Thus, the required Fourier transform does not exist for any value of the momentum $\boldsymbol{q}$ ! In Ref. [19], the $\boldsymbol{x}-\boldsymbol{x}^{\prime}$ integration is cut off at $\Lambda_{\mathrm{QCD}}^{-1}$, on the grounds that the approximations made in obtaining (2.30) are not valid at large distances. While physically reasonable, such an approach can only be applied qualitatively.

It is apparent from the discussion leading to Eq. (2.24) that Eq. (2.30) nevertheless correctly reproduces the Abelian limit at short enough distances. Explicitly,

\footnotetext{
${ }^{3}$ This expression for $\gamma$ differs from the one given in Eq. (26) of Ref. [12] by a factor of 2. This is in agreement with the requirement $\nabla^{2} \gamma(\boldsymbol{x})=\frac{1}{\boldsymbol{\nabla}^{2}} \delta^{2}(\boldsymbol{x})$.
} 


$$
\lim _{\left|\boldsymbol{x}-\boldsymbol{x}^{\prime}\right| \rightarrow 0}\left\langle A_{i}^{a}\left(x^{-}, \boldsymbol{x}\right) A_{i}^{a}\left(x^{\prime-}, \boldsymbol{x}^{\prime}\right)\right\rangle=\frac{N_{c}^{2}-1}{4 \pi} \chi\left(x^{-}, x^{\prime-}\right) \ln \left(\left|\boldsymbol{x}-\boldsymbol{x}^{\prime}\right|^{2} \Lambda_{\mathrm{QCD}}^{2}\right) .
$$

The Fourier transform of this function goes like $1 / \boldsymbol{q}^{2}$.

At larger distances, $0 \ll\left|\boldsymbol{x}-\boldsymbol{x}^{\prime}\right| \ll \Lambda_{\mathrm{QCD}}^{-1}$, and for large enough nuclei, the second term of (2.30) becomes negligible. Under these circumstances, the correlation function has a Fourier transform which behaves as $\ln \boldsymbol{q}^{2}$. To see how large the nucleus has to be to have such a regime, consider the function $f(\xi) \equiv \xi^{\Omega \xi}$. It has a minimum at $\xi=e^{-1}$, independent of $\Omega$, and a minimum value of $e^{-\Omega / e}$. Thus, to legitimately drop the second term of (2.30) we should have

$$
\Omega=\frac{N_{c} \chi}{16 \pi \Lambda_{\mathrm{QCD}}^{2}} \gg e .
$$

This constraint is difficult to satisfy: in fact, for experimentally accessible nuclei, such as uranium, we actually have $\Omega<1$. Just to obtain $\Omega \sim e$ requires $\chi \sim 45 \Lambda_{\mathrm{QCD}}^{2}$. For the model described in Sec. $\nabla$, this implies a nucleus with of order $10^{5}$ nucleons. This is a somewhat larger nucleus than is required simply to make the color charge density large enough for the approximations described at the beginning of this section to be reasonable [compare Eqs. (2.6) and (2.32)]. So while the authors of Refs. [1 , 4, 12, 19 point out that realistic nuclei are only marginally large enough, one must go to even larger nuclei to justify dropping the second term in (2.30).

Our point is, that the range of momenta where the picture described at the beginning of this section and in Refs. [12,19,23] is applicable is very narrow at best. While it is unlikely that anything short of a full non-perturbative solution to QCD can produce reliable predictions for the region $\left|\boldsymbol{x}-\boldsymbol{x}^{\prime}\right| \gtrsim \Lambda_{\mathrm{QCD}}^{-1}$, we may reasonably hope that if we construct a model which is well-behaved in this region, then we will be able to trust it closer to the boundary point $\left|\boldsymbol{x}-\boldsymbol{x}^{\prime}\right|=\Lambda_{\mathrm{QCD}}^{-1}$ than if we use Eq. (2.30), with its unphysical large-distance behavior. In the next section we will show that the source of the difficulties encountered in Eq. (2.30) is the inconsistency of the charge density correlator (2.20) with the intuitive picture of color neutral nucleons. When the correlator is altered to reflect this expectation, we find that the resulting gluon distribution is well-behaved and completely infrared finite.

\section{COLOR NEUTRALITY}

As described in the previous section, the MV model employs the Gaussian weight (2.3) for performing ensemble averages. Thus, the ensemble average of the charge denisty vanishes [1]:

$$
\left\langle\rho^{a}\left(x^{-}, \boldsymbol{x}\right)\right\rangle=0 .
$$

This says that at any given point, when averaging over all of the different nuclei within the ensemble, we see no net charge. Eq. (3.1) is gauge-invariant in the sense that if we perform the same gauge transformation on the fields present in all of the nuclei, the ensemble average remains unchanged.

Let us consider the situation inside a single hadronic state, which we will denote by $|h\rangle$. This state could consist of a single baryon or meson, or it could be some collection of hadrons. For such a state, the charge density at a given point is not necessarily zero: 


$$
\left\langle h\left|\rho^{a}\left(x^{-}, \boldsymbol{x}\right)\right| h\right\rangle \neq 0 .
$$

However, if we sum over all of the space occupied by the hadronic state, we should obtain zero:

$$
\left\langle h\left|\int d x^{-} d^{2} \boldsymbol{x} \rho^{a}\left(x^{-}, \boldsymbol{x}\right)\right| h\right\rangle=0 .
$$

Eq. (3.3) says that any physically observable hadronic state is a color singlet. Since this is true for all of the hadronic states in our Hilbert space, we may use the polarization trick to generalize $(3.3)$ to

$$
\left\langle h\left|\int d x^{-} d^{2} \boldsymbol{x} \rho^{a}\left(x^{-}, \boldsymbol{x}\right)\right| h^{\prime}\right\rangle=0 .
$$

Since we have phrased Eq. (3.4) in terms of color singlet physical states, it must hold independent of the gauge chosen for $\rho^{a}\left(x^{-}, \boldsymbol{x}\right)$.

Now consider the bilinear combination

$$
\mathcal{Z} \equiv\left\langle h\left|\int d x^{-} d^{2} \boldsymbol{x} \int d x^{\prime-} d^{2} \boldsymbol{x}^{\prime} \rho^{a}\left(x^{-}, \boldsymbol{x}\right) \rho^{b}\left(x^{\prime-}, \boldsymbol{x}^{\prime}\right)\right| h\right\rangle .
$$

On one hand, by inserting a complete set of hadronic states and applying Eq. (3.4) we conclude that $\mathcal{Z}$ vanishes. Since the ensemble utilized in the MV model consists of a specific collection of hadronic states (i.e. nuclei with some fixed number of nucleons), we further conclude that the ensemble average of $\mathcal{Z}$ vanishes. On the other hand, we may also compute the ensemble average of $\mathcal{Z}$ with the help of the correlation function (2.20):

$$
\begin{aligned}
\langle\mathcal{Z}\rangle & =\int d x^{-} d^{2} \boldsymbol{x} \int d x^{\prime-} d^{2} \boldsymbol{x}^{\prime}\left\langle\rho^{a}\left(x^{-}, \boldsymbol{x}\right) \rho^{b}\left(x^{\prime-}, \boldsymbol{x}^{\prime}\right)\right\rangle \\
& =\delta^{a b} \int d^{2} \boldsymbol{\Sigma} \int_{-\infty}^{\infty} d x^{-} \mu^{2}\left(x^{-}\right) \int d^{2} \boldsymbol{\Delta} \mathcal{D}(\boldsymbol{\Delta}) .
\end{aligned}
$$

The integral over $\boldsymbol{\Sigma}$ is just the (non-zero) transverse area of the nucleus. Since $\mu^{2}\left(x^{-}\right)$is positive (by definition), the only way to obtain a vanishing ensemble average is to require that $\mathcal{D}$ satisfy

$$
\int d^{2} \boldsymbol{\Delta} \mathcal{D}(\boldsymbol{\Delta})=0
$$

This relation is not true for the choice (2.4) employed in Ref. [12]. Hence we conclude that this choice is incompatible with the expectation of color-neutral nucleons. In fact, this violation of color-neutrality contributes to the poor infrared behavior of the MV correlation function (2.30).

Although the function $\mathcal{D}(\boldsymbol{\Delta})$ is itself not gauge-invariant (its transformation properties are determined by the condition that the Gaussian weight be gauge-invariant), the colorneutrality condition (3.7) was, nonetheless, derived in a gauge-invariant fashion, with the help of Eq. (3.4). The constraint represented by Eq. (3.7) reflects the physical observation that the nucleus does not carry a net color charge.

The fact that a color neutral charge distribution posseses an intrinsic scale explains why the infrared behavior of the gluon number density is improved by enforcing (3.7). We may 
see the roots of this result by considering Eq. (2.10), the expression for the vector potential in the covariant gauge. As pointed out earlier, it depends on an arbitrary length scale $\lambda$, because of the infrared divergence in the inverse of $\nabla^{2}$. If we integrate both sides of (2.10) over $x^{-}$and take the expectation value for the hadronic state $|h\rangle$ [so that we may apply (3.3) to the term involving $\lambda$ ] we obtain

$$
\begin{aligned}
\left\langle h\left|\int_{-\infty}^{\infty} d x^{-} \widetilde{A}^{+}\left(x^{-}, \boldsymbol{x}\right)\right| h\right\rangle & =\left\langle h\left|\int_{-\infty}^{\infty} d x^{-} \int d^{2} \boldsymbol{x}^{\prime} G\left(\boldsymbol{x}-\boldsymbol{x}^{\prime}\right) \rho\left(x^{-}, \boldsymbol{x}^{\prime}\right)\right| h\right\rangle \\
& =\frac{1}{4 \pi}\left\langle h\left|\int_{-\infty}^{\infty} d x^{-} \int d^{2} \boldsymbol{x}^{\prime} \ln \left(\boldsymbol{x}-\boldsymbol{x}^{\prime}\right)^{2} \rho\left(x^{-}, \boldsymbol{x}^{\prime}\right)\right| h\right\rangle .
\end{aligned}
$$

Instead of an arbitrary scale, the logarithm is supplied with the scale intrinsic to $\rho$, that is, the scale over which (3.3) is true.

Therefore, let us employ a charge-density correlation function $\mathcal{D}$ which satisfies (3.7). Furthermore, let us make the reasonable assumption that $\mathcal{D}$ is rotationally invariant. Under these circumstances, the function $L\left(\boldsymbol{x}-\boldsymbol{x}^{\prime}\right)$ is completely free of infrared difficulties! In this situation, we find that (2.23) may be simplified to

$$
L\left(\boldsymbol{x}-\boldsymbol{x}^{\prime}\right)=\frac{1}{16 \pi} \int d^{2} \boldsymbol{\Delta} \mathcal{D}(\boldsymbol{\Delta})\left[\left(\boldsymbol{x}-\boldsymbol{x}^{\prime}-\boldsymbol{\Delta}\right)^{2} \ln \left(\boldsymbol{x}-\boldsymbol{x}^{\prime}-\boldsymbol{\Delta}\right)^{2}-\boldsymbol{\Delta}^{2} \ln \boldsymbol{\Delta}^{2}\right] .
$$

(see Appendix B). In addition, the expression for $\mathcal{L}$ becomes

$$
\mathcal{L}\left(\boldsymbol{x}-\boldsymbol{x}^{\prime}\right)=\frac{-1}{8 \pi} \int d^{2} \boldsymbol{\Delta} \mathcal{D}(\boldsymbol{\Delta}) \ln \left(\boldsymbol{x}-\boldsymbol{x}^{\prime}-\boldsymbol{\Delta}\right)^{2}
$$

In the next section we will study the gluon number density implied by these two equations.

At various points in our subsequent discussion it will prove convenient to take the form of $\mathcal{D}(\boldsymbol{\Delta})$ to be

$$
\mathcal{D}(\boldsymbol{\Delta})=\delta^{2}(\boldsymbol{\Delta})-C(\boldsymbol{\Delta})
$$

In Sec. IVA we will see that to reproduce the Abelian limit at short distances, $\mathcal{D}(\boldsymbol{\Delta})$ must contain a delta function. Likewise, such a delta function is argued for in Refs. [1 [1, 12]. Such a term is consistent with nucleons that contain pointlike quarks. In addition, we will see that such a structure arises naturally in the model examined in Sec. V. To satisfy Eq. (3.7), it is clear that the other function appearing in (3.11) must obey

$$
\int d^{2} \boldsymbol{\Delta} C(\boldsymbol{\Delta})=1
$$

We conclude this section by quantifying the connection between $\mu^{2}\left(x^{-}\right)$and the total color charge per unit area. We begin by defining $\mathcal{X}_{\infty}$ to be the integral of $\mu^{2}\left(x^{-}\right)$over all $x^{-}$:

$$
\mathcal{X}_{\infty} \equiv \int_{-\infty}^{\infty} d x^{-} \mu^{2}\left(x^{-}\right)
$$

Then, the trace of Eq. (3.6) reads 


$$
\int d x^{-} d^{2} \boldsymbol{x} \int d x^{\prime-} d^{2} \boldsymbol{x}^{\prime}\left\langle\rho^{a}\left(x^{-}, \boldsymbol{x}\right) \rho^{a}\left(x^{\prime-}, \boldsymbol{x}^{\prime}\right)\right\rangle=\left(N_{c}^{2}-1\right) \mathcal{X}_{\infty} \pi R^{2} \int d^{2} \boldsymbol{\Delta} \mathcal{D}(\boldsymbol{\Delta}) .
$$

Imagine that, instead of a color-neutral distribution of charge satisfying (3.7), we consider a single quark. Then, it would indeed be true that $\mathcal{D}(\boldsymbol{\Delta})=\delta^{2}(\boldsymbol{\Delta})$, and the charge-squared of that quark should be given by

$$
g^{2} C_{F} \equiv\left(N_{c}^{2}-1\right) \mathcal{X}_{\infty} \pi R^{2}
$$

where $C_{F}=\left(N_{c}^{2}-1\right) / 2 N_{c}$ is the Casimir factor for $S U(N)$. Thus, we see that the color charge squared per unit area is $\left(N_{c}^{2}-1\right) \mathcal{X}_{\infty}$, and that the precise meaning of $\mu^{2}\left(x^{-}\right)$is actually the charge squared per unit area per unit thickness divided by $N_{c}^{2}-1$.

\section{GLUON NUMBER DENSITY}

We begin our discussion of the properties of the gluon number density resulting from a nucleus consisting of color-neutral nucleons by inserting (2.21) into the master formula (2.2). Employing sum and difference variables for the transverse integrations we obtain

$$
\begin{aligned}
\frac{d N}{d q^{+} d^{2} \boldsymbol{q}}=\frac{q^{+}}{2 \pi^{3}} \frac{N_{c}^{2}-1}{N_{c}} & \int d^{2} \boldsymbol{\Sigma} \int d^{2} \boldsymbol{\Delta} e^{i \boldsymbol{q} \cdot \boldsymbol{\Delta}} \frac{\mathcal{L}(\boldsymbol{\Delta})}{L(\boldsymbol{\Delta})} \\
& \times \int_{-\infty}^{\infty} d x^{-} \int_{-\infty}^{\infty} d x^{-} e^{-i q^{+}\left(x^{-}-x^{\prime-}\right)}\left\{\exp \left[N_{c} \chi\left(x^{-}, x^{-}\right) L(\boldsymbol{\Delta})\right]-1\right\} .
\end{aligned}
$$

The result of the $\boldsymbol{\Sigma}$ integration appearing in Eq. (4.1) should be interpreted as $\pi R^{2}$, the transverse area of the Lorentz-contraced nucleus. f We would like to perform the transverse integrations. All of the longitudinal dependence is contained in the function $\chi\left(x^{-}, x^{\prime-}\right)$.

Recall that within the Gaussian ansatz (2.3), the function $\chi\left(x^{-}, x^{-}\right)$is given by

$$
\chi\left(x^{-}, x^{--}\right) \equiv \int_{-\infty}^{\min \left(x^{-}, x^{--}\right)} d \xi^{-} \mu^{2}\left(\xi^{-}\right) .
$$

In order to obtain a more useful expression for $\chi$, let us define

$$
\mathcal{X}\left(x^{-}\right) \equiv \int_{-\infty}^{x^{-}} d \xi^{-} \mu^{2}\left(\xi^{-}\right)
$$

Then, we see that Eq. (4.2) has the form

$$
\chi\left(x^{-}, x^{--}\right)=\mathcal{X}\left(x^{-}\right) \Theta\left(x^{\prime-}-x^{-}\right)+\mathcal{X}\left(x^{\prime-}\right) \Theta\left(x^{-}-x^{\prime-}\right) .
$$

For convenience, let us assume that the entire nucleus is localized around some positive value of $x^{-}$. Then, we may insert Eq. (4.4) into (4.1) and do one of the longitudinal integrals in each term, with the aid of the identity

\footnotetext{
${ }^{4}$ See Appendix $\mathrm{D}$ for a more careful discussion of this point.
} 


$$
\int_{-\infty}^{\infty} d x e^{i(k+i \varepsilon) x} \Theta(x-y)=\frac{i e^{i(k+i \varepsilon) y}}{k+i \varepsilon}, \quad y>0
$$

The result is

$$
\begin{aligned}
\frac{d N}{d q^{+} d^{2} \boldsymbol{q}}=\frac{q^{+}}{2 \pi^{3}} \frac{N_{c}^{2}-1}{N_{c}} \pi & R^{2} \int d^{2} \boldsymbol{\Delta} e^{i \boldsymbol{q} \cdot \boldsymbol{\Delta}} \frac{\mathcal{L}(\boldsymbol{\Delta})}{L(\boldsymbol{\Delta})} \\
& \times \frac{2 \varepsilon}{\left(q^{+}\right)^{2}+\varepsilon^{2}} \int_{-\infty}^{\infty} d x^{-} e^{-2 \varepsilon x^{-}}\left\{\exp \left[N_{c} \mathcal{X}\left(x^{-}\right) L(\boldsymbol{\Delta})\right]-1\right\} .
\end{aligned}
$$

To get a finite nonvanishing result, the remaining integral must produce exactly one inverse power of $\varepsilon$. To see how this comes about, let us integrate the key factors of (4.6) by parts:

$$
\begin{aligned}
\frac{2 \varepsilon}{\left(q^{+}\right)^{2}+\varepsilon^{2}} \int_{-\infty}^{\infty} d x^{-} e^{-2 \varepsilon x^{-}} & \left\{\exp \left[N_{c} \mathcal{X}\left(x^{-}\right) L(\boldsymbol{\Delta})\right]-1\right\}= \\
& -\left.\frac{e^{-2 \varepsilon x^{-}}}{\left(q^{+}\right)^{2}+\varepsilon^{2}}\left\{\exp \left[N_{c} \mathcal{X}\left(x^{-}\right) L(\boldsymbol{\Delta})\right]-1\right\}\right|_{-\infty} ^{\infty} \\
& +\int_{-\infty}^{\infty} d x^{-} N_{c} \mu^{2}\left(x^{-}\right) L(\boldsymbol{\Delta}) \exp \left[N_{c} \mathcal{X}\left(x^{-}\right) L(\boldsymbol{\Delta})\right] \frac{e^{-2 \varepsilon x^{-}}}{\left(q^{+}\right)^{2}+\varepsilon^{2}}
\end{aligned}
$$

Physically, we know that for $x^{-} \rightarrow-\infty, \mathcal{X}\left(x^{-}\right)$vanishes identically, whereas for $x^{-} \rightarrow \infty$, $\mathcal{X}\left(x^{-}\right)$becomes proportional to the total charge squared per unit area.f Thus, the surface term does not contribute. We may let $\varepsilon \rightarrow 0$ in the remaining integral without mishap, since the remaining longitudinal integration will turn out to be finite. Therefore, we find that

$$
\frac{d N}{d q^{+} d^{2} \boldsymbol{q}}=\frac{N_{c}^{2}-1}{2 \pi^{3}} \pi R^{2} \frac{1}{q^{+}} \int d^{2} \boldsymbol{\Delta} e^{i \boldsymbol{q} \cdot \boldsymbol{\Delta}} \mathcal{L}(\boldsymbol{\Delta}) \int_{-\infty}^{\infty} d x^{-} \mu^{2}\left(x^{-}\right) \exp \left[N_{c} \mathcal{X}\left(x^{-}\right) L(\boldsymbol{\Delta})\right] .
$$

The definition (4.3) for $\mathcal{X}$ implies that $\mu^{2}\left(x^{-}\right)=d \mathcal{X} / d x^{-}$. Hence, we may perform the remaining longitudinal integration to obtain the result

$$
\frac{d N}{d q^{+} d^{2} \boldsymbol{q}}=\frac{N_{c}^{2}-1}{N_{c}} \frac{\pi R^{2}}{2 \pi^{3}} \frac{1}{q^{+}} \int d^{2} \boldsymbol{\Delta} e^{i \boldsymbol{q} \cdot \boldsymbol{\Delta}} \frac{\mathcal{L}(\boldsymbol{\Delta})}{L(\boldsymbol{\Delta})}\left\{\exp \left[N_{c} \mathcal{X}_{\infty} L(\boldsymbol{\Delta})\right]-1\right\} .
$$

Remarkably, the gluon number density goes like $1 / q^{+}$independent of the longitudinal charge profile! The only nuclear dependence is on the total charge squared per unit area via $\mathcal{X}_{\infty}$. This conclusion depends upon the fact that the Gaussian from chosen in (2.3) is local in $x^{-}$. To generate a dependence other than $1 / q^{+}$apparently requires non-trivial correlations in $x^{-}$. As pointed out in Ref. [12], a complete renormalization group analysis of the longitudinal dependence would generate correlations in $x^{-}$, although including such correlations was beyond the scope of that paper. Since the derivation leading to the form of the correlation function given in Eq. (2.21) depends crucially on having locality in $x^{-}$, it is clear that the inclusion of such correlations is a non-trivial problem.

\footnotetext{
${ }^{5}$ If $\mathcal{X}\left(x^{-}\right)$grows at asymptotically large values of $x^{-}$, then the total charge squared per unit area is unbounded and the integral (4.6) diverges.
} 


\section{A. Transverse Asymptotic Behavior}

The asymptotic behavior at short or long transverse distances of the gluon number density (4.9) is governed by the behavior indicated by Eqs. (3.9) and (3.10) for the functions $L(\boldsymbol{x})$ and $\mathcal{L}(\boldsymbol{x})$. Consider first the situation at short distances. It is straightforward to demonstrate that for small $x$

$$
L(\boldsymbol{x}) \sim \begin{cases}\boldsymbol{x}^{2}, & \text { if } \mathcal{D}(\boldsymbol{\Delta}) \text { is smooth at } \Delta=0 ; \\ \boldsymbol{x}^{2} \ln \boldsymbol{x}^{2}, & \text { if } \mathcal{D}(\boldsymbol{\Delta}) \text { contains } \delta^{2}(\boldsymbol{\Delta}) .\end{cases}
$$

Likewise, the short-distance form of $\mathcal{L}$ is

$$
\mathcal{L}(\boldsymbol{x}) \sim \begin{cases}\text { const. } & \text { if } \mathcal{D}(\boldsymbol{\Delta}) \text { is smooth at } \Delta=0 ; \\ \ln \boldsymbol{x}^{2}, & \text { if } \mathcal{D}(\boldsymbol{\Delta}) \text { contains } \delta^{2}(\boldsymbol{\Delta}) .\end{cases}
$$

In either case, it follows that

$$
\lim _{\boldsymbol{x} \rightarrow \mathbf{0}} L(\boldsymbol{x}) \mathcal{L}(\boldsymbol{x})=0 .
$$

This observation will be used in the next subsection where we will derive a sum rule for the integral of the gluon number density over $\boldsymbol{q}$. According to (4.11), if we want to reproduce the Abelian limit at large $\boldsymbol{q}$ with a gluon number density which goes like $1 / \boldsymbol{q}^{2}, \mathcal{D}(\boldsymbol{\Delta})$ must contain $\delta^{2}(\boldsymbol{\Delta})$. Otherwise, the necessary logarithmic divergence in the correlation function at short distances will not occur, and the gluon number density will fall off faster than in an Abelian theory.

The situation at long distances is a bit more complicated. For a rotationally symmetric correlation function $\mathcal{D}$, the color neutrality condition (3.7) may be rewritten as

$$
\int_{0}^{\infty} d \Delta \Delta \mathcal{D}(\Delta)=0
$$

Because this integral is convergent, at large distances we have either $\mathcal{D}(\Delta) \sim 1 / \Delta^{2+p}$ where $p>0$ (and could be non-integral), or else $\mathcal{D}(\Delta)$ oscillates. ๆ We will not consider the second possibility any further, as it corresponds to onion-like nucleons, with successive layers of alternating color charge all the way to infinity.

For positive $p$, we find from (3.9) and (3.10) that

$$
L(\boldsymbol{x}) \sim \begin{cases}\frac{\operatorname{sgn} \mathcal{D}}{p^{2}(2-p)^{2}} x^{2-p}, & \text { if } p \neq 2 ; \\ \frac{\operatorname{sgn} \mathcal{D}}{16} \ln ^{2} \boldsymbol{x}^{2}, & \text { if } p=2,\end{cases}
$$

where "sgn $\mathcal{D}$ " stands for the sign of $\mathcal{D}$ at large distances. The long-distance form of $\mathcal{L}$ is

\footnotetext{
${ }^{6}$ If $\mathcal{D}(\Delta)$ falls off faster than a power of $\Delta$, then the infrared behavior is even better than described in this section. The model described in Sect. $\mathrm{V}$ provides two examples of this type of correlator.
} 


$$
\mathcal{L}(\boldsymbol{x}) \sim-\frac{\operatorname{sgn} \mathcal{D}}{2 p^{2}} \frac{1}{x^{p}} .
$$

Combining these expressions into the integrand appearing in (4.9) we see that

$$
\frac{\mathcal{L}(\boldsymbol{\Delta})}{L(\boldsymbol{\Delta})}\left[\exp \left\{N_{c} \mathcal{X}_{\infty} L(\boldsymbol{\Delta})\right\}-1\right] \sim \begin{cases}\frac{(2-p)^{2}}{2 \Delta^{2}}\left\{1-\exp \left[\frac{N_{c} \mathcal{X}_{\infty} \operatorname{sgn} \mathcal{D}}{p^{2}(2-p)^{2}} \Delta^{2-p}\right]\right\}, & \text { if } p \neq 2 \\ \frac{2}{\Delta^{2} \ln ^{2} \Delta^{2}}\left\{1-\exp \left[\frac{N_{c} \mathcal{X}_{\infty} \operatorname{sgn} \mathcal{D}}{16} \ln ^{2} \Delta^{2}\right]\right\}, & \text { if } p=2 .\end{cases}
$$

Since $\mathcal{X}_{\infty}$ is proportional to the charge-squared per unit area, it is positive. Thus, we see that whether or not the gluon number density remains bounded will be determined by the sign of $\mathcal{D}(\boldsymbol{x})$ at large distances in addition to the value of $p$.

First consider the situation when $\operatorname{sgn} \mathcal{D}=-1$. Such a correlation function corresponds to the notion of a charge which is screened at large distances. If $0<p<2$, then the exponent in (4.16) goes to $-\infty$ and we are left with an integrand which falls like $1 / \Delta^{2}$. This corresponds to a gluon number density which grows like $\ln \boldsymbol{q}^{2}$ at low momenta. For $p>2$, the exponent goes to zero, and we end up with an integrand which falls like $1 / \Delta^{p}$. The Fourier transform of such a function goes like $q^{p-2}$ at small momenta. Thus, we conclude that the gluon number density is constant at small $\boldsymbol{q}$ in this situation. Finally, if $p=2$, the leading behavior of (4.16) gives an integrand which falls like $(\Delta \ln \Delta)^{-2}$, intermediate between the two previous cases. Thus, when $\operatorname{sgn} \mathcal{D}=-1$, we are guaranteed that the Fourier transform appearing in the gluon number density exists.

On the other hand, when $\operatorname{sgn} \mathcal{D}=+1$, we encounter difficulties for $0<p \leq 2$. In this situation, the integrand blows up at large distances. In order to have a physically sensible gluon number density when $\operatorname{sgn} \mathcal{D}=1$, we must have $p>2$, in which case we once again have an integrand which falls like $1 / \Delta^{p}$.

In Ref. [2], McLerran and Venugopalan argued that for $\boldsymbol{q} \rightarrow 0$, the gluon number density should approach a constant. However, as the authors of Ref. [12 correctly observe, the subtleties associated with using a $\delta$-function for the longitudinal dependence source were not correctly taken into account in Ref. [2]. Consequently, these authors claim that the correct $\boldsymbol{q} \rightarrow 0$ dependence is $\ln \boldsymbol{q}^{2}$. In our refined treatment, where the nucleons are forced to obey color-neutrality, we see that either behavior is possible, depending upon the form of the two-point charge density correlation function at large separations. If $\mathcal{D}(\Delta)$ falls off faster than $1 / \Delta^{4}$ at large distances, then the gluon number density approaches a constant as $\boldsymbol{q} \rightarrow 0$, independent of the sign of $\mathcal{D}$ in this region. On the other hand, if $\mathcal{D}(\Delta)$ falls off faster than $1 / \Delta^{2}$ but more slowly than $1 / \Delta^{4}$, we obtain a gluon number density which grows like $\ln \boldsymbol{q}^{2}$ for $\boldsymbol{q} \rightarrow 0$. In this case, we must have $\operatorname{sgn} \mathcal{D}=-1$ to obtain a physically sensible result.

Our gluon number density possesses two distinct noteworthy features at small transverse momenta. The first, as described in the previous paragraph, is the softening of the $\boldsymbol{q}$ dependence from $1 / \boldsymbol{q}^{2}$. This softening is present for all values of $\mathcal{X}_{\infty}$, and it even occurs in an Abelian theory! As we have already noted, the Abelian expression for the correlation 
function is simply proportional to $\mathcal{L}(\boldsymbol{\Delta})$. Hence, from Eq. (4.15) we see that even the longdistance Abelian correlator possesses the power-law behavior required to slow the growth of the distribution function to less than $1 / \boldsymbol{q}^{2}$ at small transverse momenta. The physical interpretation of this result is simple: a color-neutral system of size $a$ is an inefficient generator of radiation with wavelengths $\lambda \gg a$. At the end of the next section we will discuss the second noteworthy feature of our results: our distribution functions depend on $\mathcal{X}_{\infty}$ at low $\boldsymbol{q}$ in a manner consistent with the picture of gluon recombination at high densities envisioned by Gribov, Levin and Ryskin 22].

\section{B. Transverse Momentum Sum Rule}

Now that we have established the conditions under which the Fourier transform appearing in (4.9) is well-defined, let us see more explicitly how the non-Abelian corrections alter the transverse momentum dependence of the lowest order gluon number density. In the Abelian limit, Eq. (4.9) becomes

$$
\left.\frac{d N}{d q^{+} d^{2} \boldsymbol{q}}\right|_{\substack{\text { lowest } \\ \text { order }}}=\left(N_{c}^{2}-1\right) \frac{\mathcal{X}_{\infty} \pi R^{2}}{2 \pi^{3}} \frac{1}{q^{+}} \int d^{2} \boldsymbol{\Delta} e^{i \boldsymbol{q} \cdot \boldsymbol{\Delta}} \mathcal{L}(\boldsymbol{\Delta}) .
$$

Let us subtract (4.17) from (4.9) and integrate over all transverse momenta. The only $\boldsymbol{q}$-dependence contained in either expression is $e^{i \boldsymbol{q} \cdot \boldsymbol{\Delta}}$ : hence, the $\boldsymbol{q}$ integration produces $4 \pi^{2} \delta^{2}(\boldsymbol{\Delta})$. This allows us to trivially perform the $\boldsymbol{\Delta}$ integration, with the result

$$
\begin{aligned}
\int d^{2} \boldsymbol{q}\left\{\frac{d N}{d q^{+} d^{2} \boldsymbol{q}} \mid \begin{array}{c}
\text { all } \\
\text { orders }
\end{array}\right. & \left.-\left.\frac{d N}{d q^{+} d^{2} \boldsymbol{q}}\right|_{\text {lowest }}\right\}= \\
& 2 \frac{N_{c}^{2}-1}{N_{c}} \frac{R^{2}}{q^{+}} \lim _{\boldsymbol{\Delta} \rightarrow \mathbf{0}} \frac{\mathcal{L}(\boldsymbol{\Delta})}{L(\boldsymbol{\Delta})}\left\{\exp \left[N_{c} \mathcal{X}_{\infty} L(\boldsymbol{\Delta})\right]-1-N_{c} \mathcal{X}_{\infty} L(\boldsymbol{\Delta})\right\} .
\end{aligned}
$$

Since the position-space function $L(\boldsymbol{\Delta})$ vanishes at zero separation, we may evaluate the limit by expanding the exponential, producing

$$
\int d^{2} \boldsymbol{q}\left\{\left.\frac{d N}{d q^{+} d^{2} \boldsymbol{q}}\right|_{\substack{\text { all } \\ \text { orders }}}-\left.\frac{d N}{d q^{+} d^{2} \boldsymbol{q}}\right|_{\substack{\text { lowest } \\ \text { order }}}\right\}=N_{c}\left(N_{c}^{2}-1\right) \mathcal{X}_{\infty}^{2} R^{2} \frac{1}{q^{+}} \lim _{\boldsymbol{\Delta} \rightarrow \mathbf{0}} L(\boldsymbol{\Delta}) \mathcal{L}(\boldsymbol{\Delta}) .
$$

But, according to Eq. (4.12), the limit appearing on the right hand side of (4.19) vanishes. Thus, we are left with the transverse momentum sum rule

$$
\int d^{2} \boldsymbol{q}\left\{\left.\frac{d N}{d q^{+} d^{2} \boldsymbol{q}}\right|_{\substack{\text { all } \\ \text { orders }}}-\left.\frac{d N}{d q^{+} d^{2} \boldsymbol{q}}\right|_{\substack{\text { lowest } \\ \text { order }}}\right\}=0 .
$$

Eq. (4.20) states that the non-Abelian contributions have no effect on the total number of gluons: we could have obtained the same number of gluons by ignoring the non-linear terms in the vector potential. What these contributions actually do is to move gluons from one value of the transverse momentum to another. Thus, the total energy in the gluon field is 
affected by the non-Abelian terms. Note that this conclusion is independent of the form of $\mathcal{D}(\boldsymbol{\Delta})$.

If we now assume that $\mathcal{D}(\boldsymbol{\Delta})$ is of the form (3.11), with the smooth part of the correlation function $C(\boldsymbol{\Delta})$ being a positive monotonic function, we can show from Eqs. (B24) $-($ B26) that $L(\boldsymbol{\Delta})$ and $\mathcal{L}(\boldsymbol{\Delta})$ are both monotonically decreasing functions of $\Delta$. Since $L(0)=0$, this means that $L(\boldsymbol{\Delta})$ is negative. Comparing Eqs. (4.9) and (4.17) we see that the integrand for the all orders gluon number density differs from the lowest-order result by the factor

$$
\frac{\exp \left[N_{c} \mathcal{X}_{\infty} L(\boldsymbol{\Delta})\right]-1}{N_{c} \mathcal{X}_{\infty} L(\boldsymbol{\Delta})}
$$

which is less than or equal to unity for $L(\boldsymbol{\Delta}) \leq 0$. Since $L(\boldsymbol{\Delta})$ becomes more negative at large $\boldsymbol{\Delta}$, we see that the non-Abelian corrections actually serve to reduce the degree of correlations in the infrared, resulting in a depletion in the number of low- $\boldsymbol{q}$ gluons relative to the Abelian result. This depletion can be quite drastic. For example, when $p<2$, we have an Abelian distribution which diverges like $1 / \boldsymbol{q}^{2-p}$ at small $\boldsymbol{q}$ whereas the non-Abelian terms moderate this to $\ln \boldsymbol{q}^{2}$ ! On the other hand, in the ultraviolet the number of gluons is unchanged: the factor in (4.21) is very nearly 1. Hence, our transverse momentum sum rule tells us that there must be an enhancement in the number of gluons at intermediate momenta. Indeed, we will see this explicitly in the model presented in Sec. $\nabla$. Furthermore, (4.21) implies that the effect becomes more pronounced as $\mathcal{X}_{\infty}$ increases. This is consistent with the idea of saturation presented in [22], which is framed in terms of parton recombination. When $\mathcal{X}_{\infty}$ is large, gluons begin to overlap, and can readily merge via the non-Abelian terms in the equations of motion, which become more and more important in determining the correlation function (2.21) as the density is increased. If we further imagine that each source factor $\rho(\boldsymbol{x})$ contributes some characteristic amount of transverse momentum, then at the end of the day we find fewer gluons with low $\boldsymbol{q}$, and a corresponding enhancement at intermediate $\boldsymbol{q}$.

\section{Gluon Structure Functions and the DGLAP Equation}

The usual gluon structure functions resolved at the scale $Q^{2}$, namely $g_{A}\left(x_{F}, Q^{2}\right)$, may be obtained by supplying the trivial factors needed to convert $q^{+}$into $x_{F}$ and integrating Eq. (4.9) over transverse momenta less than or equal to $Q$ :

$$
\begin{aligned}
g_{A}\left(x_{F}, Q^{2}\right) & \equiv \int_{|\boldsymbol{q}| \leq Q} d^{2} \boldsymbol{q} \frac{d N}{d x_{F} d^{2} \boldsymbol{q}} \\
& =\frac{N_{c}^{2}-1}{N_{c}} \frac{\pi R^{2}}{2 \pi^{3}} \frac{1}{x_{F}} \int_{|\boldsymbol{q}| \leq Q} d^{2} \boldsymbol{q} \int d^{2} \boldsymbol{\Delta} e^{i \boldsymbol{q} \cdot \boldsymbol{\Delta}} \frac{\mathcal{L}(\boldsymbol{\Delta})}{L(\boldsymbol{\Delta})}\left\{\exp \left[N_{c} \mathcal{X}_{\infty} L(\boldsymbol{\Delta})\right]-1\right\}
\end{aligned}
$$

Suppose that we consider measuring the gluon distribution function at large $Q^{2}$. Then, we may use the transverse momentum sum rule (4.20) to replace the all-orders expression on the right hand side of (4.22) by its Abelian counterpart:

$$
g_{A}\left(x_{F}, Q^{2}\right)=\frac{1}{x_{F}} \int_{|\boldsymbol{q}| \leq Q} d^{2} \boldsymbol{q} \int d^{2} \boldsymbol{\Delta} e^{i \boldsymbol{q} \cdot \boldsymbol{\Delta}} \mathcal{L}(\boldsymbol{\Delta})\left(N_{c}^{2}-1\right) \frac{\mathcal{X}_{\infty} \pi R^{2}}{2 \pi^{3}}
$$


As explained in the discussion surrounding Eq. (3.15), the combination $\left(N_{c}^{2}-1\right) \mathcal{X}_{\infty} \pi R^{2}$ is the total charge squared of the nucleus, which for a nucleus containing $A$ nucleons is simply $3 A g^{2} C_{F}$.

Eq. (4.23) is useful because although we are unable to perform the Fourier transform appearing in (4.22) for the all-orders result with an arbitrary correlation function $\mathcal{D}(\boldsymbol{\Delta})$, we are able to do so at lowest order. Recall that $\mathcal{L}$ is the convolution of $\mathcal{D}$ with a logarithm [see Eq. (3.10)]. Its Fourier transform is just the product of the transforms of these two functions. Therefore, Eq. (4.23) simplifies to

$$
g_{A}\left(x_{F}, Q^{2}\right)=\frac{1}{x_{F}} \int_{|\boldsymbol{q}| \leq Q} d^{2} \boldsymbol{q} \frac{\widetilde{\mathcal{D}}(\boldsymbol{q})}{2 \boldsymbol{q}^{2}} \frac{3 A g^{2} C_{F}}{2 \pi^{3}} .
$$

Finally, if the correlation function is rotationally invariant, we may do the angular integration to obtain

$$
g_{A}\left(x_{F}, Q^{2}\right)=3 A C_{F} \frac{1}{x_{F}} \int_{0}^{Q^{2}} \frac{d q^{2}}{q^{2}} \widetilde{\mathcal{D}}(q) \frac{\alpha_{s}}{\pi} .
$$

Thus, for large enough values of $Q$, we see that the number of gluons at a given value of $x_{F}$ scales with the number of nucleons. Thanks to the sum rule, this statement is true to all orders in the coupling constant: any non-Abelian corrections to (4.25) vanish in the $Q^{2} \rightarrow \infty$ limit.

The expression in (4.25) is also closely connected to the Dokshitzer-Gribov-LipatovAltarelli-Parisi (DGLAP) equation [25,26]. Denoting the gluon distribution function by $g\left(x_{F}, Q^{2}\right)$, the quark distribution function by $q_{i}\left(x_{F}, Q^{2}\right)$ for flavor $i$, and the antiquark distribution function by $\bar{q}_{i}\left(x_{F}, Q^{2}\right)$, the DGLAP evolution equation for the gluon distribution function of a single nucleon reads

$$
\begin{aligned}
\frac{\partial g\left(x_{F}, Q^{2}\right)}{\partial \ln Q^{2}}=\frac{\alpha_{s}\left(Q^{2}\right)}{2 \pi} \int_{x_{F}}^{1} \frac{d \xi}{\xi} & {\left[P_{g q}\left(\frac{x}{\xi}, \alpha_{s}\left(Q^{2}\right)\right) \sum_{i}\left[q_{i}\left(\xi, Q^{2}\right)+\bar{q}_{i}\left(\xi, Q^{2}\right)\right]\right.} \\
+ & \left.P_{g g}\left(\frac{x}{\xi}, \alpha_{s}\left(Q^{2}\right)\right) g\left(\xi, Q^{2}\right)\right] .
\end{aligned}
$$

The functions $P_{g q}$ and $P_{g g}$ appearing in Eq. (4.26) are the usual Altarelli-Parisi splitting functions [26]. At lowest order, we set $g\left(\xi, Q^{2}\right)=0$ on the right hand side of (4.26), in accordance with the premise of the MV model that the gluons are generated only by the valence quarks. Thus, the only splitting function we require is (to leading order)

$$
P_{g q}(x)=C_{F}\left[\frac{1+(1-x)^{2}}{x}\right] \text {. }
$$

Inserting (4.27) into (4.26) we obtain

$$
\frac{\partial g\left(x_{F}, Q^{2}\right)}{\partial \ln Q^{2}}=C_{F} \frac{\alpha_{s}\left(Q^{2}\right)}{2 \pi} \frac{1}{x_{F}} \int_{x_{F}}^{1} d \xi\left[1+(1-x)^{2}\right] \sum_{i}\left[q_{i}\left(\xi, Q^{2}\right)+\bar{q}_{i}\left(\xi, Q^{2}\right)\right] .
$$

Since the MV model is only supposed to apply at small $x_{F}$, we take the $x_{F} \rightarrow 0$ limit of (4.28). In this limit, we end up with the sum of the quark and antiquark distribution 
functions for all flavors integrated over all values of $x_{F}$ : this is just 3 for a single nucleon. Hence

$$
\frac{\partial g\left(x_{F}, Q^{2}\right)}{\partial \ln Q^{2}}=3 C_{F} \frac{\alpha_{s}\left(Q^{2}\right)}{\pi} \frac{1}{x_{F}}
$$

We now see that the DGLAP equation at small $x_{F}$, Eq. (4.29), is very similar to the MV result of Eq. (4.25). Remarkably, the MV gluon distribution function is almost $A$ times the DGLAP result for a single nucleon. Until now, we have been ambiguous about the precise scale at which we should evaluate the strong coupling. This comparison tells us that we ought to let it run, evaluating it at $q^{2}$ and keeping it inside the integral in Eq. (4.25). By doing this, we effectively incorporate certain quantum corrections in our otherwise classical treatment. The only other difference between Eqs. (4.25) and (4.29) is the appearance of $\widetilde{\mathcal{D}}(q)$ in the MV expression. Since the color neutrality condition (3.7) also tells us that $\widetilde{\mathcal{D}}(0)=0$, we see that the presence of this factor serves to regulate the integral at small values of $q^{2}$. On the other hand, as described in the discussion following Eq. (4.11), if we believe that the nucleon should contain point-like quarks, then $\mathcal{D}(\boldsymbol{\Delta})$ contains $\delta^{2}(\boldsymbol{\Delta})$ and $\widetilde{\mathcal{D}}(q) \sim 1$ at large momenta.

\section{Average Transverse Momentum-Squared}

Because the gluon number density we have obtained in Eq. (4.9) is well-defined, we are able to explicitly compute the average transverse momentum-squared $\left\langle\boldsymbol{q}^{2}\right\rangle$ associated with this distribution. In performing this calculation, we have assumed that

$$
\mathcal{D}(\boldsymbol{\Delta})=\delta^{2}(\boldsymbol{\Delta})-C(\Delta)
$$

That is, we choose a rotationally invariant correlator which is consistent with nucleons that contain point-like quarks. Furthermore, we work to next-to-leading order in the momentum expansion, since the first non-trivial nuclear dependence enters in at that level.

As we show in Appendix Q , the gluon number density may be cast into the form

$$
\frac{d N}{d x_{F}}=\frac{N_{c}^{2}-1}{\pi^{2}} \pi R^{2} \frac{1}{x_{F}} Q^{2} \int_{-\infty}^{\infty} d x^{-} \mu^{2}\left(x^{-}\right) \int d^{2} \boldsymbol{x} \frac{J_{1}(Q x)}{Q x} \mathcal{L}(\boldsymbol{x}) e^{N_{c} \mathcal{X}\left(x^{-}\right) L(\boldsymbol{x})} .
$$

As we have remarked earlier, all of the non-Abelian effects are contained in the exponential factor. We should like some way to organize the expansion of (4.31) in powers of $1 / Q$. In the limit $Q \rightarrow \infty$, the Bessel function appearing in this expresion tends to a $\delta$-function. This suggests that the $\boldsymbol{x}$ integration will be dominated by the small $\boldsymbol{x}$ region. To connect an expansion in $\boldsymbol{x}$ with the desired expansion in $1 / Q$ we rescale the integral in (4.31) via $\boldsymbol{x} \equiv \boldsymbol{y} / Q$ :

$$
\frac{d N}{d x_{F}}=\frac{N_{c}^{2}-1}{\pi^{2}} \pi R^{2} \frac{1}{x_{F}} \int_{-\infty}^{\infty} d x^{-} \mu^{2}\left(x^{-}\right) \int d^{2} \boldsymbol{y} \frac{J_{1}(y)}{y} \mathcal{L}\left(\frac{\boldsymbol{y}}{Q}\right) \exp \left[N_{c} \mathcal{X}\left(x^{-}\right) L\left(\frac{\boldsymbol{y}}{Q}\right)\right] .
$$

In Appendix B, we argue that for a correlation function of the form (4.30), the functions $L(\boldsymbol{x})$ and $\mathcal{L}(\boldsymbol{x})$ are simply some power series in $x$ plus $\ln x^{2}$ times some other power series 
in $x$. In the case of $L(\boldsymbol{x})$ both series start at $x^{2}$ rather than $x^{0}$. Furthermore, we observe that the intrinsic length scale which must appear in the logarithms should be of order the nucleon size $a$. This same length scale serves to make the expansion parameter dimensionless. Looking at Eq. (4.32), we see that by expanding everything except for the Bessel function, it becomes apparent that the final result will consist of terms of the form $\left[1 /(a Q)^{m}\right] \ln ^{n}(a Q)^{2}$. Because the series for $L(\boldsymbol{x})$ begins at $x^{2}$, the smallest inverse power of $a Q$ which may multiply $\ln ^{n}(a Q)^{2}$ is $1 /(a Q)^{2 n-2}$. Furthermore, if we keep track of the powers of $\mathcal{X}$, we see that each additional power of $\mathcal{X}$ comes with an additional factor of $1 / Q^{2}$. Thus, the leading order term is the same as in an Abelian theory. Since $\mathcal{X} \sim A^{1 / 3}$, we conclude that the non-Abelian corrections are enhanced in very large nuclei: the subleading terms become important if $N_{c} \mathcal{X}_{\infty} \sim Q^{2}$.

We now proceed directly to our results, deferring the details of this somewhat lengthy calculation to Appendix G. At next-to-leading order, we find that

$$
\frac{d N}{d x_{F}}=\frac{1}{x_{F}} \frac{\mathcal{Z}^{2}}{4 \pi^{2}}\left[1-\frac{N_{c} \mathcal{X}_{\infty}}{4 \pi Q^{2}}\right] \ln \left(\frac{Q}{Q_{0}}\right)^{2},
$$

where

$$
\ln \left(\frac{1}{Q_{0}}\right)^{2} \equiv 2\left(\gamma_{E}-\ln 2\right)+\int d^{2} \Delta C(\Delta) \ln \Delta^{2}
$$

$\gamma_{E}$ is Euler's constant, and $\mathcal{Z}^{2}=3 A g^{2} C_{F}$ is the total charge-squared of the nucleus. As advertised, the scale appearing in the logarithm does not enter in as an additional parameter which must be determined from the data. Instead, given a particular model for implementing the color neutrality condition (3.7), we obtain a specific value for the momentum scale, Eq. (4.34). The integral appearing in Eq. (4.34) is closely related to the value of $\mathcal{L}(\boldsymbol{x})$, i.e.

$$
\int d^{2} \boldsymbol{\Delta} C(\Delta) \ln \Delta^{2}=\lim _{\boldsymbol{x} \rightarrow \mathbf{0}}\left[8 \pi \mathcal{L}(\boldsymbol{x})+\ln \boldsymbol{x}^{2}\right] .
$$

As we have already remarked in the previous subsection, at leading order in $Q, d N / d x_{F}$ simply proportional to the number of nucleons: all of the non-Abelian contributions are suppressed for $Q \rightarrow \infty$. Although at first glance the leading order contribution to Eq. (4.33) looks very different from the result presented in the previous subsection, the two expressions actually do agree. To see this, insert the Fourier integral representation for $\widetilde{\mathcal{D}}(\boldsymbol{q})$ into Eq. (4.24), perform the $\boldsymbol{q}$ integration, and subsequently integrate by parts. Calculation of the sub-leading term requires the full treatment given in Appendix $\mathbf{Q}$.

Finally, the next-to-leading order expression we obtain for $\left\langle\boldsymbol{q}^{2}\right\rangle$ reads

$$
\left\langle\boldsymbol{q}^{2}\right\rangle=\frac{Q^{2}-4 \pi C(0)}{\ln \left(Q / Q_{0}\right)^{2}}+\frac{N_{c} \mathcal{X}_{\infty}}{8 \pi}\left[\ln \left(\frac{Q}{Q_{0}}\right)^{2}-2\right] .
$$

${ }^{7}$ If $C(\Delta)$ is derived from a rotationally invariant function of the vector $\boldsymbol{\Delta}$, then the odd powers of $x$ will be absent in this expansion. 
We see that at leading order, Eq. (4.36) is independent of the size of the nucleus. A mild dependence on the modelling of the nucleons enters in through the appearance of $C(0)$. The non-Abelian terms make their first appearance at next-to-leading order. As previously, the scale of the logarithms is not arbitrary, but rather determined by the form of $C(\Delta)$, according to Eq. (4.34).

\section{A MODEL WITH COLOR-NEUTRAL NUCLEONS}

To illustrate some of the features of our improved treatment, we turn to a specific model introduced by Kovchegov [27]. In this model, we imagine a nucleus of radius $R$, containing $A$ nucleons of radius $a \sim \Lambda_{\mathrm{QCD}}^{-1}$. Each "nucleon" is made up of a quark-antiquark pair. The view in the rest frame of the nucleus is as follows: The quark and antiquark for the $i$ th nucleon is assumed to be located with equal probability anywhere within a distance $a$ of the center of the nucleon. The nucleons are assumed to be located with equal probability anywhere within a distance $R$ of the center of the nucleus. In this manner, confinement of the valence partons within the individual nucleons is ensured. For the sake of comparison, we will also present results where the quarks are distributed within the nucleons using a Gaussian weight instead of the uniform weight employed in Ref. [27].

Kovchegov begins with the laboratory frame charge density

$$
\rho\left(x^{-}, \boldsymbol{x}\right)=g \sum_{i=1}^{N}\left(T^{a}\right)\left(T_{i}^{a}\right)\left[\delta\left(x^{-}-x_{i}^{-}\right) \delta^{2}\left(\boldsymbol{x}-\boldsymbol{x}_{i}\right)-\delta\left(x^{-}-x_{i}^{\prime-}\right) \delta^{2}\left(\boldsymbol{x}^{\prime}-\boldsymbol{x}_{i}^{\prime}\right)\right]
$$

for the ultrarelativistic nucleus. In Eq. (5.1) the quark belonging to the $i$ th nucleon is located at $\left(x_{i}^{-}, \boldsymbol{x}_{i}\right)$ whereas the $i$ th antiquark is located at $\left(x_{i}^{\prime-}, \boldsymbol{x}_{i}^{\prime}\right)$. The $T^{a}$ 's are generators in the $\mathrm{SU}\left(N_{c}\right)$ color space, while the $T_{i}^{a}$ 's are similar generators in the color space of each nucleon. Kovchegov explicitly constructs the light-cone gauge vector potential generated by this distribution, and uses that result to derive an effective two-dimensional charge distribution which leads to this vector potential, i.e. he writes

$$
\rho\left(x^{-}, \boldsymbol{x}\right) \equiv \delta\left(x^{-}\right) \rho_{2}(\boldsymbol{x})
$$

and computes $\rho_{2}(\boldsymbol{x})$. From this explicit form for $\rho_{2}(\boldsymbol{x})$, one may then average over the positions of the quarks and nucleons as described above to obtain the two point correlator $\left\langle\rho_{2}^{a}(\boldsymbol{x}) \rho_{2}^{b}\left(\boldsymbol{x}^{\prime}\right)\right\rangle$. This quantity is connected to the three-dimensional correlator of Eq. (2.20) via

$$
\left\langle\rho^{a}\left(x^{-}, \boldsymbol{x}\right) \rho^{b}\left(x^{\prime-}, \boldsymbol{x}^{\prime}\right)\right\rangle=\delta\left(x^{-}-x^{\prime-}\right)\left\langle\rho_{2}^{a}(\boldsymbol{x}) \rho_{2}^{b}\left(\boldsymbol{x}^{\prime}\right)\right\rangle
$$

In this way, Kovchegov's model specifies the $\boldsymbol{x}$-dependence of the charge density correlator.

The result obtained in Ref. [27] is of the form

$$
\left\langle\rho_{2}^{a}(\boldsymbol{x}) \rho_{2}^{b}\left(\boldsymbol{x}^{\prime}\right)\right\rangle=\frac{g^{2}}{N_{c}} A \delta^{a b}\left[\mathcal{I}\left(\boldsymbol{x}, \boldsymbol{x}^{\prime}\right)-\overline{\mathcal{I}}\left(\boldsymbol{x}, \boldsymbol{x}^{\prime}\right)\right] .
$$

In Eq. (5.4), the function $\mathcal{I}\left(\boldsymbol{x}, \boldsymbol{x}^{\prime}\right)$ is the contribution that results in the averaging when a quark overlaps with a quark (or an antiquark overlaps with an antiquark), whereas $\overline{\mathcal{I}}\left(\boldsymbol{x}, \boldsymbol{x}^{\prime}\right)$ is the contribution that results when a quark overlaps with an antiquark. Explicitly, 


$$
\mathcal{I}\left(\boldsymbol{x}, \boldsymbol{x}^{\prime}\right)=\int \frac{d^{2} \boldsymbol{r} d z}{\frac{4}{3} \pi R^{3}} \int \frac{d^{2} \boldsymbol{\xi} d \zeta}{\frac{4}{3} \pi a^{3}} \delta^{2}(\boldsymbol{x}-\boldsymbol{r}-\boldsymbol{\xi}) \delta^{2}\left(\boldsymbol{x}^{\prime}-\boldsymbol{r}-\boldsymbol{\xi}\right)
$$

and

$$
\overline{\mathcal{I}}\left(\boldsymbol{x}, \boldsymbol{x}^{\prime}\right)=\int \frac{d^{2} \boldsymbol{r} d z}{\frac{4}{3} \pi R^{3}} \int \frac{d^{2} \boldsymbol{\xi} d \zeta}{\frac{4}{3} \pi a^{3}} \int \frac{d^{2} \boldsymbol{\xi}^{\prime} d \zeta^{\prime}}{\frac{4}{3} \pi a^{3}} \delta^{2}(\boldsymbol{x}-\boldsymbol{r}-\boldsymbol{\xi}) \delta^{2}\left(\boldsymbol{x}^{\prime}-\boldsymbol{r}-\boldsymbol{\xi}^{\prime}\right) .
$$

In Eqs. (5.5) and (5.6), $(\boldsymbol{r}, z)$ locate the position of the nucleon with respect to the (arbitrary) origin while $\left(\boldsymbol{\xi}^{(\prime)}, \zeta^{(\prime)}\right)$ locate the (anti)quark with respect to the center of the nucleon. Note that the $\delta$ functions are two-dimensional, whereas the integrations are three-dimensional. If we use a Gaussian instead of a uniform distribution, we should make the replacement

$$
\frac{1}{\frac{4}{3} \pi a^{3}} \rightarrow \frac{1}{\left(2 \pi a^{2}\right)^{3 / 2}} \exp \left[-\frac{\boldsymbol{\xi}^{2}+\zeta^{2}}{2 a^{2}}\right]
$$

for the quark positions. ${ }^{8}$ A detailed evaluation of all of the integrals relating to the uniform distribution is presented in Appendix D. In the Gaussian case, the integrations are straightforward, and we shall simply present the results.

The function defined by Eq. (5.5) is obviously proportional $\delta^{2}\left(\boldsymbol{x}-\boldsymbol{x}^{\prime}\right)$, the precise $\boldsymbol{x}$ dependence postulated by the authors of Ref. [12]. It is also obvious that Eq. (5.6) contains no such singular behavior: it is, in fact, a smooth function of the coordinates. For physical applications where we will be looking at scales much smaller than the nucleon radius $a$, this smooth function may be neglected in comparison to the $\delta$-function terms, as was done in Ref. [27]. However, as we noted in Sec. [II, taking only the $\delta$-function term produces a correlator which is incompatible with the idea of color-neutral nucleons. Thus, when we look at scales approaching $a$, it becomes crucial to include the contribution from Eq. (5.6). Eq. (5.4) obviously implies that $\mathcal{D}\left(\boldsymbol{x}-\boldsymbol{x}^{\prime}\right)$ has the form introduced in Eq. (3.11). By sorting out the various prefactors associated with (5.3), (5.4), and (D3), we may determine the value of $\mathcal{X}_{\infty}$ from the requirement that the two contributions to (3.11) each have unit integral. We find that

$$
\mathcal{X}_{\infty}=\frac{g^{2}}{N_{c}} \frac{A}{\pi R^{2}}
$$

Eq. (5.8) implies a total charge squared of $2 A g^{2} C_{F}$, instead of $3 A g^{2} C_{F}$ as was used in Sec. IVQ, because this model uses "nucleons" which are $q \bar{q}$ pairs instead of $q q q$ triplets. The function $C\left(\boldsymbol{x}-\boldsymbol{x}^{\prime}\right)$ which enforces color neutrality on scales near and beyond the nucleon radius is given by

$$
C\left(\boldsymbol{x}-\boldsymbol{x}^{\prime}\right)=\frac{9}{4 \pi^{2} a^{6}} \int_{|\boldsymbol{\xi}| \leq a} d^{2} \boldsymbol{\xi} \int_{\left|\boldsymbol{\xi}^{\prime}\right| \leq a} d^{2} \boldsymbol{\xi}^{\prime} \sqrt{a^{2}-\boldsymbol{\xi}^{2}} \sqrt{a^{2}-\boldsymbol{\xi}^{\prime 2}} \delta^{2}\left(\boldsymbol{x}-\boldsymbol{x}^{\prime}-\boldsymbol{\xi}+\boldsymbol{\xi}^{\prime}\right)
$$

\footnotetext{
${ }^{8}$ There is no a priori reason to assume that the parameters $a$ and $R$ appearing in the uniform and Gaussian distributions are the same. When we need to make the distinction clear, we will use a subscript $U$ on the uniform parameters and a $G$ on the Gaussian parameters.
} 


$$
=\frac{9 \Theta(1-X)}{16 \pi a^{2}}\left[\left(2+X^{2}\right) \sqrt{1-X^{2}}-X^{2}\left(4-X^{2}\right) \tanh ^{-1} \sqrt{1-X^{2}}\right]
$$

for a uniform distribution of quarks and nucleons and

$$
C\left(\boldsymbol{x}-\boldsymbol{x}^{\prime}\right)=\frac{e^{-X^{2}}}{4 \pi a^{2}}
$$

for the Gaussian distribution. In Eqs. (5.9) and (5.10) we have defined the dimensionless distance measure $X \equiv\left|\boldsymbol{x}-\boldsymbol{x}^{\prime}\right| /(2 a)$ (for details, see Appendix D). These two functions have been plotted in Fig. 11. In the uniform case, $C\left(\boldsymbol{x}-\boldsymbol{x}^{\prime}\right)$ vanishes identically for $\left|\boldsymbol{x}-\boldsymbol{x}^{\prime}\right| \geq 2 a$, whereas in the Gaussian case it has, not surprisingly, a Gaussian tail. Both functions are finite at the origin.

Now that we have specified the functional form of $\mathcal{D}(\boldsymbol{\Delta})$, we may return to Eqs. (3.9) and (3.10) and evaluate the inputs to the correlation function, $L\left(\boldsymbol{x}-\boldsymbol{x}^{\prime}\right)$ and $\mathcal{L}\left(\boldsymbol{x}-\boldsymbol{x}^{\prime}\right)$. In the uniform case we obtain

$$
\begin{aligned}
L\left(\boldsymbol{x}-\boldsymbol{x}^{\prime}\right)=\frac{a^{2}}{4 \pi} & {\left[\left(\frac{93}{100}+\frac{373}{200} X^{2}-\frac{491}{800} X^{4}-\frac{1}{64} X^{6}\right) \sqrt{1-X^{2}}\right.} \\
& \left.-\left(\frac{2}{5}+2 X^{2}-\frac{1}{4} X^{6}+\frac{1}{64} X^{8}\right) \tanh ^{-1} \sqrt{1-X^{2}}\right] \Theta(1-X) \\
-\frac{a^{2}}{20 \pi} & {\left[\frac{93}{20}+\ln \left(\frac{X^{2}}{4}\right)\right] }
\end{aligned}
$$

and

$$
\mathcal{L}\left(\boldsymbol{x}-\boldsymbol{x}^{\prime}\right)=\frac{1-X^{2}}{32 \pi}\left[\left(8+8 X^{2}-X^{4}\right) \tanh ^{-1} \sqrt{1-X^{2}}-\left(14+X^{2}\right) \sqrt{1-X^{2}}\right] \Theta(1-X) .
$$

The asymptotic forms of these functions are

$$
L\left(\boldsymbol{x}-\boldsymbol{x}^{\prime}\right) \sim \begin{cases}\frac{a^{2} X^{2}}{4 \pi}\left[\ln \left(\frac{X^{2}}{4}\right)+\frac{3}{2}\right], & X \ll 1 ; \\ -\frac{a^{2}}{20 \pi}\left[\ln \left(\frac{X^{2}}{4}\right)+\frac{93}{20}\right], & X \gg 1 ;\end{cases}
$$

and

$$
\mathcal{L}\left(\boldsymbol{x}-\boldsymbol{x}^{\prime}\right) \sim\left\{\begin{array}{cc}
-\frac{1}{8 \pi}\left[\ln \left(\frac{X^{2}}{4}\right)+\frac{7}{2}\right], & X \ll 1 \\
0 \text { (exactly), } & X \gg 1 .
\end{array}\right.
$$

Because $\mathcal{L}\left(\boldsymbol{x}-\boldsymbol{x}^{\prime}\right)$ vanishes at large distances, the Fourier transform of the correlation function (2.21) constructed from (5.11) and (5.12) will remain finite at zero (transverse) momentum. 
If instead we employ Gaussian distributions for the quarks we obtain

$$
L\left(\boldsymbol{x}-\boldsymbol{x}^{\prime}\right)=\frac{a^{2}}{4 \pi}\left[\left(1+X^{2}\right) \operatorname{Ei}\left(-X^{2}\right)+\exp \left(-X^{2}\right)-\ln \left(X^{2}\right)-\left(1+\gamma_{E}\right)\right]
$$

and

$$
\mathcal{L}\left(\boldsymbol{x}-\boldsymbol{x}^{\prime}\right)=-\frac{1}{8 \pi} \operatorname{Ei}\left(-X^{2}\right)
$$

Eqs. (5.15) and (5.16) contain the exponential integral function evaluated at negative values of the argument. We have employed the conventions of Ref. [32], i.e.

$$
\operatorname{Ei}(-z) \equiv-\int_{x}^{\infty} \frac{d t}{t} e^{-t}, \quad(z>0)
$$

Also appearing in the first of these two equations is Euler's constant $\gamma_{E}$. Asymptotically, we have

$$
L\left(\boldsymbol{x}-\boldsymbol{x}^{\prime}\right) \sim \begin{cases}\frac{a^{2} X^{2}}{4 \pi}\left[\ln \left(X^{2}\right)+\gamma_{E}-2\right], & X \ll 1 \\ -\frac{a^{2}}{4 \pi}\left[\ln \left(X^{2}\right)-\left(1+\gamma_{E}\right)\right], & X \gg 1\end{cases}
$$

and

$$
\mathcal{L}\left(\boldsymbol{x}-\boldsymbol{x}^{\prime}\right) \sim\left\{\begin{aligned}
-\frac{1}{8 \pi}\left[\ln \left(X^{2}\right)+\gamma_{E}\right], & X \ll 1 \\
\frac{1}{8 \pi} \frac{\exp \left(-X^{2}\right)}{X^{2}}, & X \gg 1
\end{aligned}\right.
$$

In this case, $\mathcal{L}\left(\boldsymbol{x}-\boldsymbol{x}^{\prime}\right)$ has an exponential tail at large distances, and we end up with a correlation function which falls like $\exp \left(-X^{2}\right) /\left[X^{2} \ln \left(X^{2}\right)\right]$ in the infrared. Again, the gluon number density will be finite at zero transverse momentum.

We have compiled a series of plots (Figs. 2 6) to aid in the comparison of the two models to each other and to the results of Ref. [12]. Each version of the correlation function effectively depends on two parameters, which we take to be $\mathcal{X}_{\infty}$ and $a_{U}, a_{G}$, or $\Lambda_{\mathrm{QCD}}$. In preparing these plots, we have adjusted these parameters so that the ultraviolet limit of (2.21) is identical for all three cases. Recall that in the ultraviolet limit, $\mathcal{X}_{\infty}$ appears multiplicatively [see Eq. (2.24)]. Thus, matching in the ultraviolet really means matching the values of $\mathcal{L}$ at short distances. From (4.17), (5.14) and (5.19), we determine that

$$
\begin{aligned}
a_{G} & =2 \exp \left(\frac{\gamma_{E}}{2}-\frac{7}{4}\right) a_{U} \\
& \approx 0.464 a_{U}
\end{aligned}
$$

and

$$
\Lambda_{\mathrm{QCD}}=\frac{1}{4} e^{7 / 4} a_{U}^{-1}
$$




$$
\approx 1.44 a_{U}^{-1}
$$

In Fig. (2), we have plotted the trace of the correlation function (2.21) in position space versus the dimensionless distance $X$. In drawing these curves, we have assumed that the longitudinal coordinates have been fixed at some value such that $a^{2} \chi\left(x^{-}, x^{\prime-}\right)=20$. Notice that the curve for the MV correlator given in Eq. (2.30) begins to diverge significantly from the other two curves near $X=0.3$. At $X \approx 0.347$, corresponding to $\left|\boldsymbol{x}-\boldsymbol{x}^{\prime}\right|=\Lambda_{\mathrm{QCD}}^{-1}$, the MV correlator vanishes. Beyond this point, it rockets off to $-\infty$ faster than any exponential. In contrast, both the uniform and Gaussian curves are well-behaved. The correlation function generated from the uniform quark/nucleon distribution vanishes for $X \geq 1$.

Next, we perform the Fourier transform to form the gluon number density, Eq. (4.9). The results are plotted in Fig. (3) for a fixed value of the longitudinal momentum $q^{+}$. In order to define the Fourier transform in the MV case, we have done what was suggested in Ref. [19] and simply cut off the $\boldsymbol{\Delta}$ integration at $\Lambda_{\mathrm{QCD}}^{-1}$. All three curves have generally the same over-all shape: a plateau at small values of $\boldsymbol{q}$, a sharp decrease at intermediate values of $\boldsymbol{q}$, and a tail for large values of $\boldsymbol{q}$. The differing values for $\boldsymbol{q} \rightarrow \mathbf{0}$ may easily understood from the position space functions in Fig. 2. The MV curve has the smallest number of zero momentum gluons because of the abrupt cut-off imposed at $\Delta=\Lambda_{\mathrm{QCD}}^{-1}$. There are more zero momentum gluons in the uniform case, since the corresponding position space function extends out to $\left|\boldsymbol{x}-\boldsymbol{x}^{\prime}\right|=2 a$. Finally, the exponential tail at large distances in the Gaussian case produces even more zero momentum gluons. At large momenta, the gluon number density is supposed to go like $1 / \boldsymbol{q}^{2}$. To illustrate this transition, we have plotted the gluon number density multiplied by $(a q)^{2}$ in Fig. 4. In this figure, the uniform and Gaussian curves are virtually indistinguishable. Beyond $(a q)^{2}=10^{4}$, they remain flat. The oscillations visible in the MV curve are a result of the sharp cutoff of the Fourier transform integral. As $(a q)^{2}$ grows, they become more rapid, but decrease in amplitude.

Next, we illustrate the effects of the non-Abelian contributions to the correlation function. For Fig. 5, we generate the ratio

$$
\frac{\left\langle A_{i}^{a}\left(x^{-}, \boldsymbol{x}\right) A_{i}^{a}\left(x^{\prime-}, \boldsymbol{x}^{\prime}\right)\right\rangle_{\text {all orders }}}{\left\langle A_{i}^{a}\left(x^{-}, \boldsymbol{x}\right) A_{i}^{a}\left(x^{\prime-}, \boldsymbol{x}^{\prime}\right)\right\rangle_{\text {lowest order }}}=\frac{e^{N_{c} \chi L\left(\boldsymbol{x}-\boldsymbol{x}^{\prime}\right)}-1}{N_{c} \chi L\left(\boldsymbol{x}-\boldsymbol{x}^{\prime}\right)} .
$$

From this expression, we see that the relative importance of the non-Abelian terms depends on the magnitude of $\chi$ : if $\chi \rightarrow 0$, then this ratio goes to 1 . Fig. 5 plots this ratio for various values of $a^{2} \chi$ ranging from 2 to 40 using the uniform version of Kovchegov's model.9 We see that the non-Abelian terms have the effect of suppressing the magnitude of the correlation functions at large distances relative to the Abelian result. In momentum space, this translates into a depletion of low momentum gluons.

Finally, we present Fig. 6. This is a plot of the all-orders gluon density (4.9) divided by the lowest-order gluon density (4.17) at fixed $q^{+}$for various values of $\mathcal{X}_{\infty}$. We see that for small values of $\boldsymbol{q}$, there are fewer gluons as $\mathcal{X}_{\infty}$ increases. At very large values of $\boldsymbol{q}$, there

\footnotetext{
${ }^{9}$ If we employ Eq. (5.8) and set $R \approx A^{1 / 3} a$, we find that a typical magnitude for $a^{2} \chi$ is approximately 2.5 for uranium.
} 
is no change. On the other hand, at small values of $\boldsymbol{q}$, this ratio is less than one, signalling that in this region the number of gluons is no longer simply proportional to the number of nucleons. The amount of suppression increases with increasing $\mathcal{X}_{\infty}$, consistent with gluon recombination scenario envisioned in Ref. [22]. Because of the transverse momentum sum rule (4.20), the area under each of the curves should be equal. Thus, we see a pile up of gluons at intermediate values of $\boldsymbol{q}$. These features of the gluon number density depend only on choosing a correlator consistent with color screening at large distances $(\operatorname{sgn} \mathcal{D}(\Delta)=-1$ for large $\Delta$ ).

We close this section by evaluating the parameters appearing in the expression for the average transverse momentum-squared within this model. According to Eq. (4.34), the value of the scale associated with the momentum logarithms may be constructed from Eqs. (5.14) and $(5.19)$ :

$$
Q_{0}=\left\{\begin{aligned}
\frac{1}{2} \exp \left(-\gamma_{E}+\frac{7}{4}\right) a_{U}^{-1} & \approx 1.615 a_{U}^{-1}, & & \text { uniform distribution } \\
\exp \left(-\frac{\gamma_{E}}{2}\right) a_{G}^{-1} & \approx 0.749 a_{G}^{-1}, & & \text { Gaussian distribution }
\end{aligned}\right.
$$

If we relate $a_{U}$ to $a_{G}$ in the manner specified by the ultraviolet matching condition (5.20), these two expressions become equal. On the other hand, the value of the smooth part of the correlation function at the origin appearing in Eq. (4.36) in the two models is

$$
4 \pi C(0)= \begin{cases}\frac{9}{2 a_{U}^{2}}, & \text { uniform distribution; } \\ \frac{1}{a_{G}^{2}}, & \text { Gaussian distribution. }\end{cases}
$$

These two quantities differ by a factor of $\frac{1}{18} \exp \left(\frac{7}{2}-\gamma_{E}\right) \approx 1.03$ when Eq. $(5.20)$ is applied.

In Figs. 7 and 8 we compare the approximate results for $d N / d x_{F}$ and $\left\langle\boldsymbol{q}^{2}\right\rangle$ obtained in Sec. IVD with "exact" curves generated by numerical integration of the full non-linear expression for the gluon number density. Since $Q_{0}$ does not depend on whether we use a uniform or Gaussian distribution for the quarks, and $C(0)$ only weakly so, we have employed the uniform distribution in these plots, since its numerical integral is more convenient to set up. Fig. 7 is a plot of $x_{F} d N / d x_{F}$ divided by the total charge-squared. In order to see more easily how the next-to-leading order approximation (4.33) is performing, we have also plotted the ratio of the approximate to exact result in the region where the approximation begins to diverge from the full all-orders value. We see that our approximation gives an excellent description of the full result for $(a Q)^{2}$ as low as about 10 when $a^{2} \mathcal{X}_{\infty}=2$. As could have been anticipated by studying Fig. 6, for $a^{2} \mathcal{X}_{\infty}=40$ our approximation begins to break down a bit sooner, at around $\left(a Q^{2}\right)=50$ or so.

Fig. 8 compares the exact and approximate curves for $\left\langle\boldsymbol{q}^{2}\right\rangle$ versus $Q^{2}$. The cases $\mathcal{X}_{\infty}=2$ and $\mathcal{X}_{\infty}=40$ differ by so little that they are virtually indistinguishable if presented on the same plot. This is a consequence of the leading behavior $\left\langle\boldsymbol{q}^{2}\right\rangle \propto Q^{2}$. For $a^{2} \mathcal{X}_{\infty}=40$ we have shown the leading order approximation as well as the complete next-to-leading order curve generated from Eq. (4.36), since this value of $\mathcal{X}_{\infty}$ is large enough for the difference between the two approximations to be visible. We have also plotted the ratio of the approximate to exact results in the "interesting" region. The behavior of the $a^{2} \mathcal{X}_{\infty}=40$ result may 
be understood in terms of Fig. 6: for large values of $\mathcal{X}_{\infty}$, there is a pile-up of gluons at intermediate values of $\boldsymbol{q}^{2}$. This effect is purely non-Abelian in nature. Now, the leading order approximation is completely independent of any of the non-Abelian contributions to the gluon number density. Thus, it is not surprising that the leading order expression falls short of the full result at moderate $Q^{2}$. The first subleading contribution happens to overcorrect by a small amount $(\sim 5 \%)$. Presumably the next term in the expansion will be negative in this region. In any event, the next-to-leading order expression is good to within a few percent for $(a Q)^{2}$ as low as about 20 for both values of $a^{2} \mathcal{X}_{\infty}$.

\section{CONCLUSIONS}

We have improved the McLerran-Venugopalan model by introducing a constraint on the charge-density correlation function to ensure that it is consistent with a nucleus which is, as a whole, color neutral. We find that imposing color-neutrality eliminates the divergences in the gluon number density present in the original MV model at small values of the transverse momentum, provided that the transverse part of the charge-density correlation function, $\mathcal{D}(\Delta)$ is rotationally invariant and falls off faster than $1 / \Delta^{4}$ at large distances. In this situation, the gluon number density approaches a constant value as $\boldsymbol{q} \rightarrow \boldsymbol{0}$. To obtain a gluon number density which goes as $\ln \boldsymbol{q}^{2}$ at small transverse momenta, we must have $\mathcal{D}(\Delta)$ fall off more slowly than $1 / \Delta^{4}$. This is permissible only if we impose the additional constraint that $\mathcal{D}(\Delta)<0$ at large distances. Then we can use a $\mathcal{D}(\Delta)$ which falls off nearly as slowly as $1 / \Delta^{2}$.

Because we have an expression which is mathematically well-behaved, we are able to demonstrate that the gluon distribution function within the MV model is proportional to $1 / x_{F}$ to all orders in the coupling constant, independent of the functional form of $\mu^{2}\left(x^{-}\right)$. This conclusion hinges upon the choice of a purely local dependence on the longitudinal distance in the charge-density correlator. The inclusion of quantum corrections [12 18] is expected to change this situation.

We have derived a transverse sum rule, Eq. (4.20), for the gluon number density. This sum rule indicates that the total number of gluons may be computed as if the theory were purely Abelian: the only effect of the non-Abelian terms is to shift gluons from one value of transverse momentum to another. As a consequence of this sum rule, we have shown that the gluon structure function in a nucleus at large $Q^{2}$ is simply given by $A$ times the result of the DGLAP equation for a single nucleon. We have shown that if we employ a charge density correlator which is consistent with charge screening at large distances, then we have saturation [22]: as the density of color charge $\mathcal{X}_{\infty}$ is increased, the number of low momentum gluons grows more slowly than the number of nucleons.

We have also presented relatively simple expressions for $d N / d x_{F}$ [Eq. (4.33)] and the mean transverse momentum-squared [Eq. (4.36)] as a function of $Q$, accurate to order $1 / Q^{2}$ and $Q^{0}$ respectively within this model. We are able to compute the scales of the logarithms in terms of a single model-dependent function $C(\Delta)$ which describes the manner in which charge neutrality is approached at scales near and beyond the nucleon size. Rather remarkably, the complicated non-linear structure of the full gluon distribution function may be understood in terms of the $Q^{2}$ expansion. That is, only the Abelian terms enter in 
at leading order in this expansion, with the non-Abelian contributions making their first appearance at next-to-leading order.

\section{ACKNOWLEDGMENTS}

This research is supported in part by the Natural Sciences and Engineering Research Council of Canada and the Fonds pour la Formation de Chercheurs et l'Aide à la Recherche of Québec. GM would like to thank Guy Moore numerous insightful discussions during the course of this work. CSL would like to thank Larry McLerran for instructive discussions about his model. 


\section{APPENDIX A: NOTATION AND CONVENTIONS}

We begin by defining the light-cone coordinates $x^{ \pm}$:

$$
x^{ \pm} \equiv \frac{x^{0} \pm x^{3}}{\sqrt{2}} .
$$

The transverse coordinates $x^{1}$ and $x^{2}$ form a two-dimensional vector which we denote simply as $\boldsymbol{x}$, without the usual subscript "T" or " $\perp$ " to avoid excessive clutter. Our metric has the signature $(-,+,+,+)$. Thus, the light-cone dot product reads $q^{\mu} x_{\mu}=-q^{+} x^{-}-q^{-} x^{+}+\boldsymbol{q} \cdot \boldsymbol{x}$, and $x_{ \pm}=-x^{\mp}$. We will think of $x^{+}$as the time coordinate, and $x^{-}$as the longitudinal distance coordinate $(z)$.

Occasionally it will be helpful to switch to sum and difference coordinates. We will employ the convention

$$
\Sigma \equiv \frac{1}{2}\left(\boldsymbol{x}+\boldsymbol{x}^{\prime}\right), \quad \boldsymbol{\Delta} \equiv \boldsymbol{x}-\boldsymbol{x}^{\prime}
$$

This asymmetric pair of definitions has the desirable property of a unit Jacobian.

The classical Yang-Mills equations are

$$
D_{\mu} F^{\mu \nu}=J^{\nu},
$$

where we have employed matrix form, i.e. $J^{\nu} \equiv T^{a} J^{a \nu}$, etc. The $T^{a}$ are the normalized Hermitian generators of $\mathrm{SU}\left(N_{c}\right)$ in the fundamental representation, satisfying $2 \operatorname{Tr}\left(T^{a} T^{b}\right)=$ $\delta^{a b}$. The covariant derivative is

$$
D_{\mu} F^{\mu \nu} \equiv \partial_{\mu} F^{\mu \nu}-i\left[A_{\mu}, F^{\mu \nu}\right]
$$

and the field strength is defined in terms of the potential as

$$
F^{\mu \nu} \equiv \partial^{\mu} A^{\nu}-\partial^{\nu} A^{\mu}-i\left[A^{\mu}, A^{\nu}\right] \text {. }
$$

These definitions assume that the coupling constant $g$ has been absorbed into $J^{\nu}$ [see, for example, Eq. (5.1)]. Note that this is not the same convention as employed by MV in Refs. [1] 4,12$]$, where they choose to explicitly extract the factor of $g$ from the current. We define the charge density $\rho\left(x^{-}, \boldsymbol{x}\right)$ appearing in Eq. (2.1) such that the total charge $\mathcal{Z}$ of the nucleus is given by

$$
\mathcal{Z} \equiv \int d x^{-} d^{2} \boldsymbol{x} \rho\left(x^{-}, \boldsymbol{x}\right)
$$

Because our intuitive picture of the parton model is most simply realized in the light-cone gauge [31], we elect to work primarily in that gauge. The light-cone gauge is defined by the condition

$$
A^{+}(x) \equiv 0
$$

However, solution of the classical Yang-Mills equations for the type of source we consider is simplest in the covariant (Lorentz) gauge. For clarity, gauge-dependent quantities will be written with a tilde in the covariant gauge: $e . g$. $\widetilde{A}$. The covariant gauge is specified by

$$
\partial_{\mu} \widetilde{A}^{\mu}(x) \equiv 0 .
$$




\section{APPENDIX B: THE FUNCTION $L\left(\boldsymbol{x}-\boldsymbol{x}^{\prime}\right)$}

\section{General Color-Neutral Correlator}

In this appendix we present the calculation of $L\left(\boldsymbol{x}-\boldsymbol{x}^{\prime}\right)$ for a general correlator $\mathcal{D}(\boldsymbol{\Delta})$. Our starting point is Eq. (2.23) written in terms of the sum and difference variables defined as in (A2):

$$
\begin{aligned}
L\left(\boldsymbol{x}-\boldsymbol{x}^{\prime}\right) \equiv \int d^{2} \boldsymbol{\Delta} \int d^{2} \boldsymbol{\Sigma} \mathcal{D}(\boldsymbol{\Delta}) & {\left[G\left(\boldsymbol{x}-\boldsymbol{\Sigma}-\frac{1}{2} \boldsymbol{\Delta}\right) G\left(\boldsymbol{x}^{\prime}-\boldsymbol{\Sigma}+\frac{1}{2} \boldsymbol{\Delta}\right)\right.} \\
& -\frac{1}{2} G\left(\boldsymbol{x}-\boldsymbol{\Sigma}-\frac{1}{2} \boldsymbol{\Delta}\right) G\left(\boldsymbol{x}-\boldsymbol{\Sigma}+\frac{1}{2} \boldsymbol{\Delta}\right) \\
& \left.-\frac{1}{2} G\left(\boldsymbol{x}^{\prime}-\boldsymbol{\Sigma}-\frac{1}{2} \boldsymbol{\Delta}\right) G\left(\boldsymbol{x}^{\prime}-\boldsymbol{\Sigma}+\frac{1}{2} \boldsymbol{\Delta}\right)\right],
\end{aligned}
$$

All three terms of (B1) may be evaluated by considering the integral

$$
\mathcal{S}\left(\boldsymbol{x}, \boldsymbol{x}^{\prime}, \boldsymbol{\Delta}\right) \equiv \frac{1}{16 \pi^{2}} \int d^{2} \boldsymbol{\Sigma} \ln \left(\frac{\left|\boldsymbol{x}-\boldsymbol{\Sigma}-\frac{1}{2} \boldsymbol{\Delta}\right|^{2}}{\lambda^{2}}\right) \ln \left(\frac{\left|\boldsymbol{x}^{\prime}-\boldsymbol{\Sigma}+\frac{1}{2} \boldsymbol{\Delta}\right|^{2}}{\lambda^{2}}\right) .
$$

To deal with the logarithms, we insert the identity

$$
\ln \left(\frac{B}{A}\right)=\int_{0}^{\infty} \frac{d z}{z}\left(e^{-A z}-e^{-B z}\right) .
$$

Because we will ultimately insert our result for $\mathcal{S}$ into (B1), we may drop any terms which do not depend on $\boldsymbol{\Delta}$, thanks to the charge neutrality condition (3.7). Thus, the $\lambda$-dependent bits of (B2) do not contribute, and we are left with

$\mathcal{S}\left(\boldsymbol{x}, \boldsymbol{x}^{\prime}, \boldsymbol{\Delta}\right)=\frac{1}{16 \pi^{2}} \int_{0}^{\infty} \frac{d a}{a} \int_{0}^{\infty} \frac{d b}{b} \int d^{2} \boldsymbol{\Sigma} \exp \left[-a\left(\boldsymbol{\Sigma}-\boldsymbol{x}+\frac{1}{2} \boldsymbol{\Delta}\right)^{2}-b\left(\boldsymbol{\Sigma}-\boldsymbol{x}^{\prime}-\frac{1}{2} \boldsymbol{\Delta}\right)^{2}\right]$.

The $\boldsymbol{\Sigma}$ integration is now Gaussian, and easily performed, yielding

$$
\begin{aligned}
\mathcal{S}\left(\boldsymbol{x}, \boldsymbol{x}^{\prime}, \boldsymbol{\Delta}\right)=\frac{1}{16 \pi} \int_{0}^{\infty} \frac{d a}{a} \int_{0}^{\infty} \frac{d b}{b} \frac{1}{a+b} \exp \{ & -a\left(\boldsymbol{x}-\frac{1}{2} \boldsymbol{\Delta}\right)^{2}-b\left(\boldsymbol{x}^{\prime}+\frac{1}{2} \boldsymbol{\Delta}\right)^{2} \\
+ & \left.\frac{\left[a\left(\boldsymbol{x}-\frac{1}{2} \boldsymbol{\Delta}\right)+b\left(\boldsymbol{x}^{\prime}+\frac{1}{2} \boldsymbol{\Delta}\right)\right]^{2}}{a+b}\right\} .
\end{aligned}
$$

The next step is to insert

$$
1=\int_{0}^{\infty} \frac{d \tau}{\tau} \delta\left(1-\frac{a+b}{\tau}\right)
$$

and rescale $a \rightarrow \tau a, b \rightarrow \tau b$. This allows us to simplify the exponent, producing

$\mathcal{S}\left(\boldsymbol{x}, \boldsymbol{x}^{\prime}, \boldsymbol{\Delta}\right)=\frac{1}{16 \pi} \int_{0}^{\infty} \frac{d \tau}{\tau^{2}} \int_{0}^{\infty} \frac{d a}{a} \int_{0}^{\infty} \frac{d b}{b} \delta(1-a-b) \exp \left\{-\tau a(1-a)\left(\boldsymbol{x}-\boldsymbol{x}^{\prime}-\boldsymbol{\Delta}\right)^{2}\right\}$.

The $b$ integration is now trivial, and the $a$ integration becomes trivial after the variable change 


$$
\tau=\frac{u}{a(1-a)}
$$

Thus, we obtain

$$
\mathcal{S}\left(\boldsymbol{x}, \boldsymbol{x}^{\prime}, \boldsymbol{\Delta}\right)=\frac{1}{16 \pi} \int_{0}^{\infty} \frac{d u}{u^{2}} \exp \left\{-u\left(\boldsymbol{x}-\boldsymbol{x}^{\prime}-\boldsymbol{\Delta}\right)^{2}\right\}
$$

which, when inserted into (B1) yields

$$
L\left(\boldsymbol{x}-\boldsymbol{x}^{\prime}\right)=\frac{1}{16 \pi} \int_{0}^{\infty} \frac{d u}{u^{2}} \int d^{2} \boldsymbol{\Delta} \mathcal{D}(\boldsymbol{\Delta})\left[e^{-u\left(\boldsymbol{x}-\boldsymbol{x}^{\prime}-\boldsymbol{\Delta}\right)^{2}}-e^{-u \boldsymbol{\Delta}^{2}}\right] .
$$

Although the $u$ integration in $(\overline{\mathrm{B} 10})$ appears to be divergent, for a rotationally invariant correlation function $\mathcal{D}$, the integral actually is finite. To see this, note that for small $u$ the integrand of (B10) looks like

$$
\frac{d u}{u}\left[2\left(\boldsymbol{x}-\boldsymbol{x}^{\prime}\right) \cdot \boldsymbol{\Delta}-\left(\boldsymbol{x}-\boldsymbol{x}^{\prime}\right)^{2}\right] \mathcal{D}(\boldsymbol{\Delta})+\text { finite terms. }
$$

The term containing $\left(\boldsymbol{x}-\boldsymbol{x}^{\prime}\right)^{2}$ vanishes when we perform the $\boldsymbol{\Delta}$ integration, because of (3.7). If $\mathcal{D}$ is rotationally invariant, then the angular part of the $\boldsymbol{\Delta}$ integration will cause the $\left(\boldsymbol{x}-\boldsymbol{x}^{\prime}\right) \cdot \boldsymbol{\Delta}$ term to vanish as well.

Because the integral over $u$ is finite, we may employ a trick reminiscent of dimensional regulation and write

$$
L\left(\boldsymbol{x}-\boldsymbol{x}^{\prime}\right)=\frac{1}{16 \pi} \int d^{2} \boldsymbol{\Delta} \int_{0}^{\infty} \frac{d u}{u^{2-\epsilon}} \mathcal{D}(\boldsymbol{\Delta})\left[e^{-u\left(\boldsymbol{x}-\boldsymbol{x}^{\prime}-\boldsymbol{\Delta}\right)^{2}}-e^{-u \boldsymbol{\Delta}^{2}}\right],
$$

where we understand that the limit $\epsilon \rightarrow 0$ is to be taken on the right hand side as soon as it is safe to do so. The $u$ integration may be performed by using the analytic continuation of

$$
\int_{0}^{\infty} \frac{d u}{u^{\kappa+1}}\left[e^{-\beta u}-e^{-\alpha u}\right]=\frac{1}{\kappa} \Gamma(1-\kappa)\left[\alpha^{\kappa}-\beta^{\kappa}\right]
$$

(Eq. (3.434.1) of Ref. [32]). After applying (B13) to (B12) and performing some algebra we obtain

$$
\begin{aligned}
L\left(\boldsymbol{x}-\boldsymbol{x}^{\prime}\right) & =\frac{1}{16 \pi} \frac{1}{\epsilon} \frac{\Gamma(1-\epsilon)}{1-\epsilon} \int d^{2} \boldsymbol{\Delta} \mathcal{D}(\boldsymbol{\Delta})\left[2 \boldsymbol{\Delta} \cdot\left(\boldsymbol{x}-\boldsymbol{x}^{\prime}\right)-\left(\boldsymbol{x}-\boldsymbol{x}^{\prime}\right)^{2}\right] \\
& +\frac{1}{16 \pi} \int d^{2} \boldsymbol{\Delta} \mathcal{D}(\boldsymbol{\Delta})\left[\left(\boldsymbol{x}-\boldsymbol{x}^{\prime}-\boldsymbol{\Delta}\right)^{2} \ln \left(\boldsymbol{x}-\boldsymbol{x}^{\prime}-\boldsymbol{\Delta}\right)^{2}-\boldsymbol{\Delta}^{2} \ln \boldsymbol{\Delta}^{2}\right]
\end{aligned}
$$

The pole term is the manifestation of the behavior described in (B11). For a rotationally invariant function $\mathcal{D}(\boldsymbol{\Delta})$, it vanishes. Thus, we are left with just

$$
L\left(\boldsymbol{x}-\boldsymbol{x}^{\prime}\right)=\frac{1}{16 \pi} \int d^{2} \boldsymbol{\Delta} \mathcal{D}(\boldsymbol{\Delta})\left[\left(\boldsymbol{x}-\boldsymbol{x}^{\prime}-\boldsymbol{\Delta}\right)^{2} \ln \left(\boldsymbol{x}-\boldsymbol{x}^{\prime}-\boldsymbol{\Delta}\right)^{2}-\boldsymbol{\Delta}^{2} \ln \boldsymbol{\Delta}^{2}\right]
$$

Differentiation of $(\overline{B 15})$ to obtain explicit expressions for the two functions defined in Eq. (2.25) produces 


$$
\mathcal{L}\left(\boldsymbol{x}-\boldsymbol{x}^{\prime}\right)=\frac{-1}{8 \pi} \int d^{2} \boldsymbol{\Delta} \mathcal{D}(\boldsymbol{\Delta}) \ln \left(\boldsymbol{x}-\boldsymbol{x}^{\prime}-\boldsymbol{\Delta}\right)^{2}
$$

and

$$
\mathcal{L}_{i j}\left(\boldsymbol{x}-\boldsymbol{x}^{\prime}\right)=\frac{-1}{4 \pi} \int d^{2} \boldsymbol{\Delta} \mathcal{D}(\boldsymbol{\Delta}) \frac{\left(x-x^{\prime}-\Delta\right)_{i}\left(x-x^{\prime}-\Delta\right)_{j}}{\left(\boldsymbol{x}-\boldsymbol{x}^{\prime}-\boldsymbol{\Delta}\right)^{2}} .
$$

Recall that $\mathcal{L}_{i j}$ was defined to be the traceless piece of $\partial_{i} \partial_{j}^{\prime} L\left(\boldsymbol{x}-\boldsymbol{x}^{\prime}\right)$. It does not contribute to the gluon number density.

\section{Rotationally Invariant Correlator}

If $\mathcal{D}(\boldsymbol{\Delta})$ is rotationally invariant, we may perform the angular integration appearing in Eq. (B15). Letting $\psi$ be the angle between $\boldsymbol{x}$ and $\boldsymbol{\Delta}$ we have

$$
\begin{aligned}
L(\boldsymbol{x})=\frac{1}{16 \pi} \int_{0}^{\infty} d \Delta \Delta \mathcal{D}(\Delta) & \\
& \times \int_{0}^{2 \pi} d \psi\left[\left(x^{2}-2 x \Delta \cos \psi+\Delta^{2}\right) \ln \left(x^{2}-2 x \Delta \cos \psi+\Delta^{2}\right)-\Delta^{2} \ln \Delta^{2}\right]
\end{aligned}
$$

The non-trivial angular integrals appearing in this expression are

$$
\int_{0}^{2 \pi} d \psi \ln \left(x^{2}-2 x \Delta \cos \psi+\Delta^{2}\right)= \begin{cases}2 \pi \ln x^{2} & \text { if } x>\Delta ; \\ 2 \pi \ln \Delta^{2} & \text { if } x<\Delta,\end{cases}
$$

and

$$
\int_{0}^{2 \pi} d \psi \cos \psi \ln \left(x^{2}-2 x \Delta \cos \psi+\Delta^{2}\right)= \begin{cases}-2 \pi \Delta / x & \text { if } x>\Delta \\ -2 \pi x / \Delta & \text { if } x<\Delta .\end{cases}
$$

Eqs. (B19) and (B20) force us to split up the radial integration into two pieces:

$$
\begin{aligned}
L(\boldsymbol{x}) & =\frac{1}{8} \int_{0}^{x} d \Delta \Delta \mathcal{D}(\Delta)\left[\left(x^{2}+\Delta^{2}\right) \ln x^{2}-\Delta^{2} \ln \Delta^{2}+2 \Delta^{2}\right] \\
& +\frac{x^{2}}{8} \int_{x}^{\infty} d \Delta \Delta \mathcal{D}(\Delta)\left[\ln \Delta^{2}+2\right] .
\end{aligned}
$$

Similarly, we may perform the angular integration appearing in (B16) to obtain

$$
\mathcal{L}(\boldsymbol{x})=-\frac{1}{4} \int_{0}^{x} d \Delta \Delta \mathcal{D}(\Delta) \ln x^{2}-\frac{1}{4} \int_{x}^{\infty} d \Delta \Delta \mathcal{D}(\Delta) \ln \Delta^{2}
$$

Let us further assume that $\mathcal{D}(\boldsymbol{\Delta})=\delta^{2}(\boldsymbol{\Delta})-C(\Delta)$, where $C(\Delta)$ is smooth at the origin. The color-neutrality requirement implies that

$$
\int d^{2} \Delta C(\Delta)=1, \quad \text { or } \quad \int_{0}^{\infty} d \Delta \Delta C(\Delta)=\frac{1}{2 \pi}
$$

Making this ansatz allows us to rewrite Eqs. (B21) and (B22) as 


$$
L(\boldsymbol{x})=-\frac{1}{8 \pi} x^{2}+\frac{1}{16 \pi} x^{2} \ln \left(\frac{x^{2}}{\Xi^{2}}\right)+\frac{1}{8} \int_{0}^{x} d \Delta \Delta C(\Delta)\left[\left(x^{2}+\Delta^{2}\right) \ln \left(\frac{\Delta^{2}}{x^{2}}\right)+2\left(x^{2}-\Delta^{2}\right)\right]
$$

and

$$
\mathcal{L}(\boldsymbol{x})=-\frac{1}{8 \pi} \ln \left(\frac{x^{2}}{\Xi^{2}}\right)+\frac{1}{4} \int_{0}^{x} d \Delta \Delta C(\Delta) \ln \left(\frac{x^{2}}{\Delta^{2}}\right)
$$

where

$$
\ln \Xi^{2} \equiv 2 \pi \int_{0}^{\infty} d \Delta \Delta C(\Delta) \ln \Delta^{2} .
$$

Because of Eq. (B23), the integral defining the length scale $\Xi$ in Eq. (B26) will be finite for any reasonable function $C(\Delta)$. Furthermore, the value of $\Xi$ will be closely tied to the scale implicit in $C(\Delta)$, namely the nucleon size $a \sim \Lambda_{\mathrm{OCD}}^{-1}$. Since we have stipulated that $C(\Delta)$ be smooth at $\Delta=0$, we conclude that Eqs. (B24) and (B25) are telling us that $L(\boldsymbol{x})$ and $\mathcal{L}(\boldsymbol{x})$ may be recast in the form of a power series in $x$ plus $\ln x^{2}$ times a power series in $x$. Furthermore, the power series for $\mathcal{L}(\boldsymbol{x})$ begins at $x^{0}$, whereas the first non-vanishing contribution to the series for $L(\boldsymbol{x})$ is $x^{2}$. This observation will be useful in organizing the computation of the mean transverse momentum-squared associated with our gluon distribution.

\section{APPENDIX C: COMPUTATION OF THE AVERAGE TRANSVERSE MOMENTUM-SQUARED}

In this appendix we will outline the computation of the value of $\left\langle\boldsymbol{q}^{2}\right\rangle$, the average transverse momentum-squared for the gluon distribution (4.9). By definition

$$
\left\langle\boldsymbol{q}^{2}\right\rangle \equiv \frac{\int_{|\boldsymbol{q}| \leq Q} d^{2} \boldsymbol{q} \boldsymbol{q}^{2} \frac{d N}{d x_{F} d^{2} \boldsymbol{q}}}{\int_{|\boldsymbol{q}| \leq Q} d^{2} \boldsymbol{q} \frac{d N}{d x_{F} d^{2} \boldsymbol{q}}} .
$$

We will do our computation through next-to-leading order. Since the numerator of (C1) grows like $Q^{2}$ in the limit $Q \rightarrow \infty$, this means we work to order $Q^{0}$. Likewise, since the denominator of (C1) grows like $\ln Q^{2}$ for large $Q$, we determine it through order $1 / Q^{2}$.

For the purposes of this calculation we will assume that $\mathcal{D}(\boldsymbol{\Delta})$ is of the form

$$
\mathcal{D}(\boldsymbol{\Delta})=\delta^{2}(\boldsymbol{\Delta})-C(\Delta),
$$

where the function $C(\Delta)$ is smooth at the origin. As discussed in Sec. $\mathbb{V} \mathrm{A}, \mathcal{D}(\boldsymbol{\Delta})$ should contain $\delta^{2}(\boldsymbol{\Delta})$ if the results are to agree with an Abelian theory in the ultraviolet. This is consistent with having point-like quarks in the nucleons. Beyond specifying that $C(\Delta)$ is rotationally invariant and has unit integral [to satisfy (3.7)], we will not make any additional assumptions about this function. 


\section{Calculation of $\left\langle\boldsymbol{q}^{2}\right\rangle d N / d x_{F}$ to Order $Q^{0}$}

We begin with the numerator of (C1), employing Eq. (4.8) for the integrand, since it has a simpler transverse structure than Eq. (4.9). Thus, our starting point is

$$
\left\langle\boldsymbol{q}^{2}\right\rangle \frac{d N}{d x_{F}}=\frac{N_{c}^{2}-1}{2 \pi^{3}} \pi R^{2} \frac{1}{x_{F}} \int_{-\infty}^{\infty} d x^{-} \mu^{2}\left(x^{-}\right) \int d^{2} \boldsymbol{x} \int_{|\boldsymbol{q}| \leq Q} d^{2} \boldsymbol{q} \boldsymbol{q}^{2} e^{i \boldsymbol{q} \cdot \boldsymbol{x}} \mathcal{L}(\boldsymbol{x}) e^{N_{c} \mathcal{X}\left(x^{-}\right) L(\boldsymbol{x})} .
$$

If we were to evaluate the $\boldsymbol{q}$ integral as it stands, we would obtain a mildly complicated combination of Bessel and Lommel functions. Alternatively, we may replace $\boldsymbol{q}^{2} e^{i \boldsymbol{q} \cdot \boldsymbol{x}}$ by $-\boldsymbol{\nabla}^{2} e^{i \boldsymbol{q} \cdot \boldsymbol{x}}$. Then, the momentum integration appearing in (C3) is easily performed with the help of Ref. [32]:

$$
\int_{|\boldsymbol{q}| \leq Q} d^{2} \boldsymbol{q} e^{i \boldsymbol{q} \cdot \boldsymbol{x}}=2 \pi Q^{2} \frac{J_{1}(Q x)}{Q x} .
$$

Observe that if the $\boldsymbol{q}$ integration were to extend to infinity instead of being cut off at $Q$, then the integral appearing in Eq. (C4) would have produced a delta function instead of a Bessel function. Hence, we conclude that

$$
\lim _{Q \rightarrow \infty} Q^{2} \frac{J_{1}(Q x)}{Q x}=2 \pi \delta^{2}(\boldsymbol{x}) .
$$

Eq. (C5) will serve as a useful diagnostic tool in determining the order in $Q$ of each contribution as it is encountered. That is, if the application of Eq. (C5) to a given expression results in a finite result, we will conclude that there are no contributions to that expression which grow as $Q \rightarrow \infty$. On the other hand, if the result of applying Eq. (C5) is divergent, then

we will have to do the integral involving the Bessel function exactly for finite $Q$, ensuring that we correctly determine not only the contributions which grow as $Q \rightarrow \infty$, but also the subleading terms as well.

After inserting (C4) into (C3), we integrate by parts to shift $\nabla^{2}$ off of the Bessel function. Thus, we arrive at

$$
\left\langle\boldsymbol{q}^{2}\right\rangle \frac{d N}{d x_{F}}=-\frac{N_{c}^{2}-1}{\pi^{2}} \pi R^{2} \frac{1}{x_{F}} Q^{2} \int_{-\infty}^{\infty} d x^{-} \mu^{2}\left(x^{-}\right) \int d^{2} \boldsymbol{x} \frac{J_{1}(Q x)}{Q x} \nabla^{2}\left[\mathcal{L}(\boldsymbol{x}) e^{N_{c} \mathcal{X}\left(x^{-}\right) L(\boldsymbol{x})}\right] .
$$

The derivatives appearing in Eq. (C6) yield

$$
\begin{aligned}
\nabla^{2}\left[\mathcal{L}(\boldsymbol{x}) e^{\left.N_{c} \mathcal{X}\left(x^{-}\right) L(\boldsymbol{x})\right]=\{}\right. & {\left[\boldsymbol{\nabla}^{2} \mathcal{L}(\boldsymbol{x})\right] } \\
& +N_{c} \mathcal{X}\left(x^{-}\right) \mathcal{L}(\boldsymbol{x})\left[\boldsymbol{\nabla}^{2} L(\boldsymbol{x})\right] \\
& +2 N_{c} \mathcal{X}\left(x^{-}\right)\left[\partial_{i} \mathcal{L}(\boldsymbol{x})\right]\left[\partial_{i} L(\boldsymbol{x})\right] \\
& \left.+N_{c}^{2} \mathcal{X}^{2}\left(x^{-}\right) \mathcal{L}(\boldsymbol{x})\left[\partial_{i} L(\boldsymbol{x})\right]^{2}\right\} e^{N_{c} \mathcal{X}\left(x^{-}\right) L(\boldsymbol{x})}
\end{aligned}
$$

The next steps are straightforward but tedious. Basically we have to examine each term bitby-bit and do the $\boldsymbol{x}$ integration either after applying Eq. (C5) (for the constant contributions) 
or by doing the actual integral with the Bessel function intact (for the contributions that grow as $Q \rightarrow \infty)$. Rather than go through all of this mathematics in detail, let us only mention the highlights.

The first term in Eq. (C7), up to the ubiquitous exponential factor, is purely Abelian in origin. Differentiation of Eq. (3.10) tells us that

$$
\nabla^{2} \mathcal{L}(\boldsymbol{x})=-\frac{1}{2} \mathcal{D}(\boldsymbol{x})
$$

Thus, the contribution from this term is especially easy to compute, as there is no $\boldsymbol{\Delta}$ integration. The singular part of $\mathcal{D}(\boldsymbol{x})$ leads to a contribution proportional to $Q^{2}$, whereas the smooth part produces a constant piece proportional to $C(0)$.

The second and third terms of (C7) are purely non-Abelian in origin. At lowest order (i.e. with the exponential set to unity), they are quadratic in the charge-squared. At the end of the day, the longitudinal dependence of these terms reads

$$
\begin{aligned}
\int_{-\infty}^{\infty} d x^{-} \mu^{2}\left(x^{-}\right) \mathcal{X}\left(x^{-}\right) & =\int_{-\infty}^{\infty} d x^{-} \int_{-\infty}^{x^{-}} d \xi^{-} \mu^{2}\left(x^{-}\right) \mu^{2}\left(\xi^{-}\right) \\
& =\frac{1}{2} \mathcal{X}_{\infty}^{2}
\end{aligned}
$$

Thus, we see the result will be independent of the functional form of $\mu^{2}\left(x^{-}\right)$, as would have been manifest from the beginning had we chosen to begin our calculation with Eq. (4.9) instead of Eq. (4.8).

Because $\nabla^{2} L(\boldsymbol{x})=-2 \mathcal{L}(\boldsymbol{x})$, the second term of (C7) contains $\mathcal{L}^{2}(\boldsymbol{x})$. As a consequence, we encounter the transverse integrals

$$
\mathcal{T}_{1} \equiv \int d^{2} \boldsymbol{x} \frac{J_{1}(Q x)}{Q x} \ln ^{2} \boldsymbol{x}^{2}
$$

and

$$
\mathcal{T}_{2} \equiv \int d^{2} \boldsymbol{x} \frac{J_{1}(Q x)}{Q x} \ln \boldsymbol{x}^{2} \ln (\boldsymbol{x}-\boldsymbol{\Delta})^{2}
$$

The first of these two integrals reduces to

$$
\mathcal{T}_{1}=\frac{8 \pi}{Q} \int_{0}^{\infty} d x J_{1}(Q x) \ln ^{2} x
$$

This integral may be evaluated by observing that Eq. (6.561.14) of Ref. [32] yields the identity

$$
\int_{0}^{\infty} d x J_{1}(Q x) x^{\varepsilon}=\frac{1}{Q}\left(\frac{Q}{2}\right)^{-\varepsilon} \frac{\Gamma(1+\varepsilon / 2)}{\Gamma(1-\varepsilon / 2)} .
$$

Because $\varepsilon$ is arbitrary, we may expand both sides of Eq. (C13) and equate corresponding powers of $\varepsilon$ to read off the integral of $J_{1}(Q x)$ times a logarithm to an arbitrary positive integer power. In the case of immediate interest we have 


$$
\mathcal{T}_{1}=\frac{2 \pi}{Q^{2}}\left[\ln \left(\frac{Q^{2}}{4}\right)+2 \gamma_{E}\right]^{2}
$$

We deal with $\mathcal{T}_{2}$ by first writing

$$
\mathcal{T}_{2}=2 \int d^{2} \boldsymbol{x} \frac{J_{1}(Q x)}{Q x}[\ln (Q x)-\ln Q] \ln (\boldsymbol{x}-\boldsymbol{\Delta})^{2} .
$$

Next, we rescale $\boldsymbol{x} \equiv \boldsymbol{y} / Q$ in the first term to arrive at a form which possesses a transparent $Q \rightarrow \infty$ limit:

$$
\begin{aligned}
\mathcal{T}_{2} & =2 \int d^{2} \boldsymbol{y} \frac{J_{1}(y)}{y} \ln y \ln \left(\frac{\boldsymbol{y}}{Q}-\boldsymbol{\Delta}\right)^{2} \\
& -\ln Q^{2} \int d^{2} \boldsymbol{x} \frac{J_{1}(Q x)}{Q x} \ln (\boldsymbol{x}-\boldsymbol{\Delta})^{2} .
\end{aligned}
$$

In the first term of (C16), we may neglect $\boldsymbol{y} / Q$ in the logarithm and do the remaining integral with the aid of Eq. (6.772.2) of Ref. [32]. In the second term we simply apply (C5). The result reads

$$
\mathcal{T}_{2}=-\frac{2 \pi}{Q^{2}}\left[\ln \left(\frac{Q^{2}}{4}\right)+2 \gamma_{E}\right] \ln \Delta^{2} .
$$

The third term of Eq. (C7) contains among its contributions only one new non-trivial transverse integral:

$$
\mathcal{T}_{3} \equiv \int d^{2} \boldsymbol{x} \frac{J_{1}(Q x)}{Q x} \frac{\boldsymbol{x} \cdot \boldsymbol{\Delta}}{\boldsymbol{x}^{2}} \ln (\boldsymbol{x}-\boldsymbol{\Delta})^{2} .
$$

We obtain the large- $Q$ limit of (C18) by rescaling $\boldsymbol{x} \equiv \boldsymbol{y} / Q$ and writing

$$
\ln \left(\boldsymbol{\Delta}-\frac{\boldsymbol{y}}{Q}\right)^{2}=\ln \boldsymbol{\Delta}^{2}-\frac{2 \boldsymbol{y} \cdot \boldsymbol{\Delta}}{Q \boldsymbol{\Delta}^{2}}+\mathcal{O}\left(\frac{1}{Q^{2}}\right)
$$

Then, (C18) becomes

$$
\begin{aligned}
\mathcal{T}_{3} & =\frac{1}{Q} \ln \boldsymbol{\Delta}^{2} \int d^{2} \boldsymbol{y} \frac{J_{1}(y)}{y} \frac{\boldsymbol{y} \cdot \boldsymbol{\Delta}}{\boldsymbol{y}^{2}} \\
& -\frac{2}{Q^{2}} \int d^{2} \boldsymbol{y} \frac{J_{1}(y)}{y} \frac{(\boldsymbol{y} \cdot \boldsymbol{\Delta})^{2}}{\boldsymbol{y}^{2}} .
\end{aligned}
$$

The first term of (C20) posseses a vanishing angular integral, while the second term is straightforward to evaluate. Thus,

$$
\mathcal{T}_{3}=-\frac{2 \pi}{Q^{2}}
$$

Finally, we remark that the fourth term of Eq. (C7), which consists of contributions which are cubic and higher in the charge density squared vanishes in the large- $Q$ limit. 
Essentially, this comes about because the small- $\boldsymbol{x}$ behavior of $\partial_{i} L(\boldsymbol{x})$ is $x_{i}\left[1+\ln \boldsymbol{x}^{2}\right]$. Thus, the factor $\left[\partial_{i} L(\boldsymbol{x})\right]^{2}$ contains sufficient powers of $\boldsymbol{x}$ in this region to kill off the diverging logarithms.

After working through all of the necessary algebra, we arrive at the surprisingly simple result

$$
\begin{aligned}
\left\langle\boldsymbol{q}^{2}\right\rangle \frac{d N}{d x_{F}}=\frac{1}{x_{F}} \frac{\mathcal{Z}^{2}}{4 \pi^{2}}\left[Q^{2}-4\right. & \pi C(0)] \\
+\frac{1}{x_{F}} \frac{\mathcal{Z}^{2}}{4 \pi^{2}} \frac{N_{c} \mathcal{X}_{\infty}}{8 \pi}\{ & {\left[\ln \left(\frac{Q^{2}}{4}\right)+2 \gamma_{E}+\int d^{2} \Delta C(\Delta) \ln \Delta^{2}\right]^{2} } \\
& \left.-2\left[\ln \left(\frac{Q^{2}}{4}\right)+2 \gamma_{E}+\int d^{2} \Delta C(\Delta) \ln \Delta^{2}\right]-2\right\},
\end{aligned}
$$

where we have denoted the total charge-squared of the nucleus $\left(N_{c}^{2}-1\right) \mathcal{X}_{\infty} \pi R^{2}$ by $\mathcal{Z}^{2}$. Each occurence of $\ln Q^{2}$ in Eq. (C22) comes with the same combination of $\gamma_{E}$ and integral over $C(\Delta)$, leading to the definition of $Q_{0}$ presented in Eq. (4.34).

\section{Calculation of $d N / d x_{F}$ to Order $1 / Q^{2}$}

We now turn to the denominator of (C1). Previously, we presented Eq. (4.24), which writes this quantity in terms of the momentum space representation of the charge density correlation function $\widetilde{\mathcal{D}}(\boldsymbol{q})$. However, since the numerator has been expressed in terms of the position space function $C(\Delta)$, it will be useful to do the same for the denominator as well. Besides, Eq. (4.24) is correct only to leading order, and we require the $1 / Q^{2}$ terms as well.

To obtain such an expression, we again employ Eq. (4.8) for the integrand:

$$
\frac{d N}{d x_{F}}=\frac{N_{c}^{2}-1}{2 \pi^{3}} \pi R^{2} \frac{1}{x_{F}} \int_{-\infty}^{\infty} d x^{-} \mu^{2}\left(x^{-}\right) \int d^{2} \boldsymbol{x} \int_{|\boldsymbol{q}| \leq Q} d^{2} \boldsymbol{q} e^{i \boldsymbol{q} \cdot \boldsymbol{x}} \mathcal{L}(\boldsymbol{x}) e^{N_{c} \mathcal{X}\left(x^{-}\right) L(\boldsymbol{x})} .
$$

The momentum integration appearing in (C23) may be done by applying Eq. (C4), producing

$$
\frac{d N}{d x_{F}}=\frac{N_{c}^{2}-1}{\pi^{2}} \pi R^{2} \frac{1}{x_{F}} Q^{2} \int_{-\infty}^{\infty} d x^{-} \mu^{2}\left(x^{-}\right) \int d^{2} \boldsymbol{x} \frac{J_{1}(Q x)}{Q x} \mathcal{L}(\boldsymbol{x}) e^{N_{c} \mathcal{X}\left(x^{-}\right) L(\boldsymbol{x})} .
$$

Since we are working to order $1 / Q^{2}$, it is not enough to simply apply Eq. (C5) to replace the Bessel function by a delta function when it is safe to do so, since this would make order $1 / Q^{2}$ errors. Instead we will have to expand the exponential and apply an assortment of scaling arguments to determine the order of each contribution. We will denote the contribution to $d N / d x_{F}$ which contains $m$ powers of $N_{c} \mathcal{X}\left(x^{-}\right) L(\boldsymbol{x})$ by $d N^{(m)} / d x_{F}$.

At zeroth order in this expansion we have

$$
\frac{d N^{(0)}}{d x_{F}}=-\frac{\mathcal{Z}^{2}}{8 \pi^{3}} \frac{1}{x_{F}} Q^{2}\left\{\int d^{2} \boldsymbol{x} \frac{J_{1}(Q x)}{Q x} \ln \boldsymbol{x}^{2}-\int d^{2} \boldsymbol{\Delta} C(\Delta) \int d^{2} \boldsymbol{x} \frac{J_{1}(Q x)}{Q x} \ln (\boldsymbol{x}-\boldsymbol{\Delta})^{2}\right\} .
$$


where we have used Eqs. (3.10) and (C2) to write out $\mathcal{L}(\boldsymbol{x})$. The $\boldsymbol{x}$-integration in the first term of (C25) may be performed exactly with the aid of Eq. (6.772.2) of Ref. [32]:

$$
\int d^{2} \boldsymbol{x} \frac{J_{1}(Q x)}{Q x} \ln \boldsymbol{x}^{2}=-\frac{2 \pi}{Q^{2}}\left[\ln \left(\frac{Q^{2}}{4}\right)+2 \gamma_{E}\right] .
$$

The second term, which will combine with the logarithm in (C26) to make its argument dimensionless, requires a bit more work. To this end we define

$$
\mathcal{T}_{4} \equiv Q^{2} \int d^{2} \boldsymbol{\Delta} C(\Delta) \int d^{2} \boldsymbol{x} \frac{J_{1}(Q x)}{Q x} \ln (\boldsymbol{x}-\boldsymbol{\Delta})^{2} .
$$

The rescaling $\boldsymbol{x} \equiv \boldsymbol{w} \Delta$ produces

$$
\begin{aligned}
\mathcal{T}_{4} & =Q \int d^{2} \boldsymbol{\Delta} C(\Delta) \Delta \ln \Delta^{2} \int d^{2} \boldsymbol{w} \frac{J_{1}(w Q \Delta)}{w} \\
& +Q \int d^{2} \boldsymbol{\Delta} C(\Delta) \Delta \int d^{2} \boldsymbol{w} \frac{J_{1}(w Q \Delta)}{w} \ln (\boldsymbol{w}-\hat{\boldsymbol{\Delta}})^{2}
\end{aligned}
$$

where $\hat{\boldsymbol{\Delta}}$ is a unit vector in the $\boldsymbol{\Delta}$ direction. We may immediately perform the $\boldsymbol{w}$ integration exactly in the first term of (C28). In the second term, we rescale, $\boldsymbol{\Delta} \equiv \boldsymbol{\Delta}^{\prime} /(w Q)$ :

$$
\begin{aligned}
\mathcal{T}_{4} & =\int d^{2} \boldsymbol{\Delta} C(\Delta) \ln \Delta^{2} \\
& +\frac{1}{Q^{2}} \int d^{2} \boldsymbol{w} \int d^{2} \boldsymbol{\Delta}^{\prime} C\left(\frac{\Delta^{\prime}}{w Q}\right) \Delta^{\prime} J_{1}\left(\Delta^{\prime}\right) \frac{1}{w^{4}} \ln (\boldsymbol{w}-\hat{\boldsymbol{\Delta}})^{2}
\end{aligned}
$$

The only angular dependence in the second term is contained in the logarithm. Thus, Eq. (B19) may be applied to yield

$$
\begin{aligned}
\mathcal{T}_{4} & =\int d^{2} \Delta C(\Delta) \ln \Delta^{2} \\
& +\frac{2 \pi}{Q^{2}} \int_{1}^{\infty} \frac{d w}{w^{3}} \ln w^{2} \int d^{2} \Delta^{\prime} C\left(\frac{\Delta^{\prime}}{w Q}\right) \Delta^{\prime} J_{1}\left(\Delta^{\prime}\right) .
\end{aligned}
$$

Thanks to the theta-function from Eq. (B19), the radial part of the $w$ integration begins at $w=1$, not $w=0$. If we now expand the correlation function in powers of $\Delta^{\prime} /(w Q)$, the $w$ integration will always converge. Likewise, we may always do the $\Delta^{\prime}$ integration via the analytic continuation of Eq. (6.561.14) of Ref. [32]. Thus, we obtain a well-defined expansion of the second term of (C30) in powers of $1 / Q$. In particular, the $1 / Q^{2}$ term vanishes, since

$$
\int_{0}^{\infty} d \Delta \Delta^{2} J_{1}(\Delta)=0
$$

Thus, only the first term of Eq. (C30) contributes through order $1 / Q^{2}$. Combining this result with Eqs. (C25) and (C26) we arrive at

$$
\frac{d N^{(0)}}{d x_{F}}=\frac{1}{x_{F}} \frac{\mathcal{Z}^{2}}{4 \pi^{2}}\left[\ln \left(\frac{Q^{2}}{4}\right)+2 \gamma_{E}+\int d^{2} \Delta C(\Delta) \ln \Delta^{2}\right] .
$$


The next term in the expansion of the exponential of Eq. (C24) will produce contributions of order $1 / Q^{2}$. Our overview of this part of the calculation begins with the result of doing the expansion, which reads

$$
\frac{d N^{(1)}}{d x_{F}} \equiv \frac{\mathcal{Z}^{2}}{2 \pi^{2}} N_{c} \mathcal{X}_{\infty} \frac{1}{q^{+}} Q^{2} \int d^{2} \boldsymbol{x} \frac{J_{1}(Q x)}{Q x} \mathcal{L}(\boldsymbol{x}) L(\boldsymbol{x})
$$

Eqs. (3.9) and (3.10) when combined with Eq. (C2) tell us that there are three kinds of contributions to Eq. (C33). The first is generated by multiplying the $\delta$ function containing terms of $\mathcal{L}(\boldsymbol{x})$ and $L(\boldsymbol{x})$. It may be evaluated exactly with the help of

$$
\int_{0}^{\infty} d x x^{2} \ln ^{2} x J_{1}(Q x)=\frac{2}{Q^{3}}\left[\ln \left(\frac{Q^{2}}{4}\right)+2 \gamma_{E}-1\right],
$$

which was obtained via the same methods used to integrate Eq. (C12).

The second type of contribution to Eq. (C33) consists of terms produced by combining a $\delta$ function from one factor $[\mathcal{L}(\boldsymbol{x})$ or $L(\boldsymbol{x})]$ with the smooth part of the other. Generally, these contributions are handled in the same fashion as $\mathcal{T}_{4}$ [Eqs. (C27)-(C30)]. In particular, we extract the $1 / Q^{2}$ terms via the rescaling $\boldsymbol{x}=\boldsymbol{w} \Delta$ followed by $\boldsymbol{\Delta}=\boldsymbol{\Delta}^{\prime} /(w Q)$. The only complication that arises is the appearance of the integral

$$
\mathcal{T}_{6} \equiv-\frac{Q}{4 \pi} \int d^{2} \boldsymbol{\Delta} C(\Delta) \Delta^{3} \int d^{2} \boldsymbol{w} \frac{\boldsymbol{w} \cdot \hat{\boldsymbol{\Delta}}}{w} J_{1}(w Q \Delta) \ln (w \Delta)^{2} \ln (\boldsymbol{w}-\hat{\boldsymbol{\Delta}})^{2} .
$$

The angular part of the $\boldsymbol{w}$ integration is governed by Eq. (B20), leading to

$$
\mathcal{T}_{6}=\frac{Q}{2} \int d^{2} \Delta C(\Delta) \Delta^{3} \int_{0}^{\infty} d w J_{1}(w Q \Delta) \ln (w \Delta)^{2}\left[\Theta(w-1)-w^{2} \Theta(1-w)\right]
$$

We may apply our usual rescaling of $\boldsymbol{\Delta}$ to the first term of (C36) to deduce that it is of order $\left(\ln Q^{2}\right) / Q^{4}$, and so may be neglected. However, in the second term, we cannot rescale $\Delta$ in this manner since the $w$ integration would diverge. Instead, we write $w \equiv v /(Q \Delta)$, yielding

$$
\mathcal{T}_{6}=\frac{1}{2 Q^{2}} \int d^{2} \Delta C(\Delta) \int_{0}^{Q \Delta} d v v^{2}\left(\ln v^{2}-\ln Q^{2}\right) J_{1}(v) .
$$

If we let the upper limit of the $v$ integration go to infinity, we see that the integral remains finite. Therefore, we conclude that

$$
\mathcal{T}_{6}=-\frac{2}{Q^{2}}
$$

correct through order $1 / Q^{2}$.

The final type of contribution to (C33) which we encounter is generated by multiplying the smooth parts of both factors, leading to the formidible-looking integral

$$
\int d^{2} \boldsymbol{x} \frac{J_{1}(Q x)}{Q x} \int d^{2} \boldsymbol{\Delta} C(\Delta)\left[(\boldsymbol{x}-\boldsymbol{\Delta})^{2} \ln \left(\boldsymbol{x}-\boldsymbol{\Delta}^{2}\right)-\Delta^{2} \ln \Delta^{2}\right] \int d^{2} \boldsymbol{\Delta}^{\prime} C\left(\Delta^{\prime}\right) \ln \left(\boldsymbol{x}-\boldsymbol{\Delta}^{\prime}\right)^{2} .
$$


By employing the rescalings $\boldsymbol{\Delta} \equiv \overline{\boldsymbol{\Delta}} x$ and $\boldsymbol{\Delta}^{\prime} \equiv \overline{\boldsymbol{\Delta}}^{\prime} x$ followed by $\boldsymbol{x} \equiv \boldsymbol{y} / Q$, it is straightforward to show that the leading contribution to (C39) is of order $\left(\ln ^{2} Q^{2}\right) / Q^{6}$.

At the end of this lengthy procedure we arrive at

$$
\frac{d N^{(1)}}{d x_{F}}=-\frac{1}{x_{F}} \frac{\mathcal{Z}^{2}}{4 \pi^{2}} \frac{N_{c} \mathcal{X}_{\infty}}{4 \pi Q^{2}}\left[\ln \left(\frac{Q^{2}}{4}\right)+2 \gamma_{E}+\int d^{2} \Delta C(\Delta) \ln \Delta^{2}\right] .
$$

When combined with Eq. (C32), we obtain the result (4.33) presented in Sec. IVD.

\section{APPENDIX D: THE INTEGRALS APPEARING IN KOVCHEGOV'S MODEL}

\section{The Charge Density Correlation Function}

In Sec. $\mathrm{V}$, we wrote the charge density correlation function derived from Kovchegov's model [27] in terms of the two integrals $\mathcal{I}$ and $\overline{\mathcal{I}}$, given by Eqs. (5.5) and (5.6) respectively. In this Appendix, we will explicitly evaluate these expressions assuming that the nuclear radius $R$ is much larger than the nucleon radius $a$.

The easier of the two integrals to evaluate is $\mathcal{I}$. Because the nucleus and nucleons are spherical and we assume a sharp cut-off in the allowed positions of the nucleons and the quarks, we have

$$
\mathcal{I}\left(\boldsymbol{x}, \boldsymbol{x}^{\prime}\right)=\frac{9}{4} \frac{\delta^{2}\left(\boldsymbol{x}-\boldsymbol{x}^{\prime}\right)}{\pi^{2} R^{3} a^{3}} \int_{|\boldsymbol{\xi}| \leq a} d^{2} \boldsymbol{\xi} \int_{|\boldsymbol{r}| \leq R} d^{2} \boldsymbol{r} \sqrt{R^{2}-\boldsymbol{r}^{2}} \sqrt{a^{2}-\boldsymbol{\xi}^{2}} \delta^{2}(\boldsymbol{x}-\boldsymbol{r}-\boldsymbol{\xi}) .
$$

Now an exact evaluation of (D1) would require that we satisfy the $\delta$-function by setting $\boldsymbol{r} \equiv \boldsymbol{x}-\boldsymbol{\xi}$ and integrating over only those values of $\boldsymbol{\xi}$ which are compatible with this.

However, since we take $R \gg a$, we may approximate by setting $\boldsymbol{r}^{2} \approx \boldsymbol{x}^{2}$ in the integrand and performing the $\boldsymbol{\xi}$ integration over the entire disk of radius $a$. By doing this, we are allowing nucleons which are located near the edge of the nucleus to "pop out" by a (small) amount of order $a$. In this approximation, a straightforward evaluation yields

$$
\mathcal{I}(\boldsymbol{\Sigma}, \boldsymbol{\Delta})=\frac{3}{2 \pi R^{2}} \sqrt{1-\frac{\boldsymbol{\Sigma}^{2}}{R^{2}}} \Theta\left(R^{2}-\boldsymbol{\Sigma}^{2}\right) \delta^{2}(\boldsymbol{\Delta}) .
$$

where we have switched to the sum and difference variables (A2). Now Eq. (D2) depends upon $\boldsymbol{\Sigma}$ whereas the expressions employed in Sec. $\mathrm{V}$ do not. The $\boldsymbol{\Sigma}$-dependence of Eq. (D2) is not really surprising, since the pancake obtained by Lorentz-contracting a spherical nucleus should be thinner near the edges than near the center. What we call $\mathcal{X}_{\infty}$ in Eq. (5.8) is obtained from (D2) by first integrating over $\Sigma$ : i.e. instead of simply writing $\pi R^{2}$ for the $\Sigma$ integral, as was done in the discussion following Eq. (4.1), we perform that integral including the geometric factors indicated in (D2):

$$
\int d^{2} \boldsymbol{\Sigma} \mathcal{I}(\boldsymbol{\Sigma}, \boldsymbol{\Delta})=\delta^{2}(\boldsymbol{\Delta})
$$

Comparing this result with Eqs. (5.3) and (5.4), we conclude that 


$$
\pi R^{2} \mathcal{X}_{\infty}=\frac{g^{2} N}{N_{c}}
$$

The other integral is somewhat more difficult to evaluate. Doing the longitudinal integrations and making the $R \gg a$ approximation described above yields

$$
\begin{aligned}
\overline{\mathcal{I}}(\boldsymbol{\Sigma}, \boldsymbol{\Delta})= & \frac{27}{\pi^{3} R^{2} a^{6}} \sqrt{1-\frac{\Sigma^{2}}{R^{2}}} \Theta\left(R^{2}-\boldsymbol{\Sigma}^{2}\right) \\
& \times \int_{|\boldsymbol{\xi}| \leq a} d^{2} \boldsymbol{\xi} \int_{\left|\boldsymbol{\xi}^{\prime}\right| \leq a} d^{2} \boldsymbol{\xi}^{\prime} \sqrt{a^{2}-\boldsymbol{\xi}^{2}} \sqrt{a^{2}-\boldsymbol{\xi}^{\prime 2}} \delta^{2}\left(\boldsymbol{\Delta}-\boldsymbol{\xi}+\boldsymbol{\xi}^{\prime}\right) \\
\equiv & \frac{3}{2 \pi R^{2}} \sqrt{1-\frac{\Sigma^{2}}{R^{2}}} \Theta\left(R^{2}-\boldsymbol{\Sigma}^{2}\right) C(\boldsymbol{\Delta})
\end{aligned}
$$

where $C$ is the function introduced in Eq. (5.9). Since we have defined $C(\boldsymbol{\Delta})$ by extracting the same $\boldsymbol{\Sigma}$-dependent prefactor which appeared in (D2), at the end of the day we find that $\mathcal{D}(\boldsymbol{\Delta})=\delta^{2}(\boldsymbol{\Delta})-C(\boldsymbol{\Delta})$, as written in Eq. (3.11).

A direct evaluation of the integral defining $C$ is difficult because of the $\delta$-function constraint: we are instructed to consider the area of overlap between two disks of radius $a$ separated by a distance $\Delta$. This area must be weighted by the product of the distances from the two centers to the integration point (see Fig. 9). Although this sounds simple, the only immediate conclusion which may be drawn directly from the integral is that when $|\boldsymbol{\Delta}| \geq 2 a$ the function vanishes: the disks do not overlap.

To deal with the situation when $|\boldsymbol{\Delta}| \leq 2 a$, we begin by computing the Fourier transform of $C(\boldsymbol{\Delta})$ :

$$
\begin{aligned}
\widetilde{C}(\boldsymbol{q}) & \equiv \int d^{2} \boldsymbol{\Delta} e^{i \boldsymbol{q} \cdot \boldsymbol{\Delta}} C(\boldsymbol{\Delta}) \\
& =\frac{9}{4 \pi^{2} a^{6}} \int_{|\boldsymbol{\xi}| \leq a} d^{2} \boldsymbol{\xi} \int_{\left|\boldsymbol{\xi}^{\prime}\right| \leq a} d^{2} \boldsymbol{\xi}^{\prime} \int d^{2} \boldsymbol{\Delta} e^{i \boldsymbol{q} \cdot \boldsymbol{\Delta}} \sqrt{a^{2}-\boldsymbol{\xi}^{2}} \sqrt{a^{2}-\boldsymbol{\xi}^{\prime 2}} \delta^{2}\left(\boldsymbol{\Delta}-\boldsymbol{\xi}+\boldsymbol{\xi}^{\prime}\right)
\end{aligned}
$$

Since the $\boldsymbol{\Delta}$ integration is over all space, the $\delta$-function constraint can be satisfied for all values of $\boldsymbol{\xi}$ and $\boldsymbol{\xi}^{\prime}$ : no messy $\Theta$-functions are introduced. In fact, the entire expression factorizes:

$$
\widetilde{C}(\boldsymbol{q})=\left\{\frac{3}{2 \pi a^{3}} \int_{|\boldsymbol{\xi}| \leq a} d^{2} \boldsymbol{\xi} e^{i \boldsymbol{q} \cdot \boldsymbol{\xi} \sqrt{a^{2}-\boldsymbol{\xi}^{2}}}\right\}^{2} .
$$

Performing the angular integration and rescaling the radial integral to unit range we obtain

$$
\widetilde{C}(\boldsymbol{q})=\left\{3 \int_{0}^{1} d v v \sqrt{1-v^{2}} J_{0}(a q v)\right\}^{2} .
$$

The radial integral may be performed with the help of Ref. 32 (c.f. Eq. (6.567.1)):

$$
\tilde{C}(\boldsymbol{q})=\left\{3 \sqrt{\frac{\pi}{2}} \frac{J_{3 / 2}(a q)}{(a q)^{3 / 2}}\right\}^{2} .
$$


Happily, the half-integer Bessel functions are expressible as trigonometric polynomials. Thus, we arrive finally at

$$
\widetilde{C}(\boldsymbol{q})=\frac{9}{(a q)^{6}}[\sin (a q)-(a q) \cos (a q)]^{2} .
$$

In spite of the apparent high-order singularity at $q=0, \tilde{C}(0)$ is nevertheless finite:

$$
\lim _{q \rightarrow 0} \widetilde{C}(\boldsymbol{q})=1
$$

It is amusing to note that the function implied by (D11) for the Fourier transform of $\mathcal{D}$, namely

$$
\widetilde{\mathcal{D}}(\boldsymbol{q})=1-\frac{9}{(a q)^{6}}[\sin (a q)-(a q) \cos (a q)]^{2} .
$$

is exactly the same function that arises in position space for the parallel spin pair correlation function in a noninteracting gas of spin- $\frac{1}{2}$ fermions [33]. In the Fermi gas, the role of the sphere of radius a containing a uniform distribution of quarks is played by the momentumspace filling of energy levels up to the Fermi surface. The condition that the nucleons be color neutral on large scales leads to the vanishing of $\mathcal{D}$ at zero momentum. The Pauli exclusion principle leads to the vanishing of the parallel spin pair correlation function at zero separation. ${ }^{10}$

Returning to the issue at hand, to obtain $C(\boldsymbol{\Delta})$, we simply invert the Fourier transform. The angular integration is straightforward, and leaves us with

$$
C(\boldsymbol{\Delta})=\frac{9}{4 \pi a^{2}} \int_{0}^{\infty} d q J_{0}\left(\frac{q \Delta}{a}\right)\left[\frac{1}{q^{3}}+\frac{1}{q^{5}}+\frac{\cos 2 q}{q^{3}}-\frac{2 \sin 2 q}{q^{4}}-\frac{\cos 2 q}{q^{5}}\right] .
$$

Each of the five terms on the right-hand-side of (D13) is individually divergent. In order to perform the $q$ integration term-by-term, we must insert a regulator. Since the final combination of terms in (D13) is guaranteed to be finite by (D11), the result will be insensitive to the details of the regulator employed. A convenient means of regulation is to make the replacement

$$
J_{0}\left(\frac{q \Delta}{a}\right) \rightarrow J_{2 \epsilon}\left(\frac{q \Delta}{a}\right)
$$

in (D13). We may then apply Eqs. (6.561.14), (6.699.1), and (6.699.2) of Ref. [32] to obtain

$$
C(\boldsymbol{\Delta})=\frac{9}{4 \pi a^{2}}\left\{-\frac{X^{2}}{2} \frac{1}{\epsilon} \frac{1}{1-\epsilon^{2}}+\frac{X^{4}}{8} \frac{1}{\epsilon} \frac{1}{\left(1-\epsilon^{2}\right)\left(1-\epsilon^{2} / 4\right)}\right.
$$

\footnotetext{
${ }^{10}$ The opposite spin pair correlation function for the Fermi gas is unity (the exclusion principle does not affect particles of opposite spin). This case is analagous to the non-charge conserving correlator of Eq. (2.4).
} 


$$
\begin{aligned}
-2\left(\frac{X^{2}}{4}\right)^{\epsilon} & \frac{\cos \pi \epsilon}{\epsilon(1-2 \epsilon)(2-2 \epsilon)(3-2 \epsilon)(4-2 \epsilon)} \\
\times & {\left[(3-2 \epsilon)(4-2 \epsilon){ }_{2} F_{1}\left(-1+\epsilon,-\frac{1}{2}+\epsilon ; 1+2 \epsilon ; X^{2}\right)\right.} \\
& -4(4-2 \epsilon){ }_{2} F_{1}\left(-1+\epsilon,-\frac{3}{2}+\epsilon ; 1+2 \epsilon ; X^{2}\right) \\
& \left.\left.+4{ }_{2} F_{1}\left(-2+\epsilon,-\frac{3}{2}+\epsilon ; 1+2 \epsilon ; X^{2}\right)\right]\right\},
\end{aligned}
$$

which is valid for $\Delta \leq 2 a$. In this expression we have defined the dimensionless distance $X \equiv \Delta /(2 a)$. The hypergeometric functions may be expanded, and all of the poles in $\epsilon$ cancelled. The resulting series may then be converted back into a different (generalized) hypergeometric function. The result of this tedious algebra is

$$
C(\boldsymbol{\Delta})=\frac{9}{64 \pi a^{2}}\left[2 X^{2}\left(4-X^{2}\right) \ln \left(\frac{X^{2}}{4}\right)+8+X^{4}-\frac{X^{6}}{2}{ }_{3} F_{2}\left(1,1, \frac{5}{2} ; 3,4 ; X^{2}\right)\right] .
$$

Finally, we notice that all but one of the parameters in the hypergeometric function appearing in (D16) are integers. Hence, we may eliminate this hypergeometric function by combining Eqs. (7.512.12) and (9.111) of Ref. 32] to obtain

$$
\begin{aligned}
{ }_{3} F_{2}(a, b, c ; p, q ; z)= & \frac{\Gamma(p) \Gamma(q)}{\Gamma(b) \Gamma(p-b) \Gamma(c) \Gamma(q-c)} \\
& \quad \times \int_{0}^{1} d t \int_{0}^{1} d u t^{b-1}(1-t)^{p-b-1} u^{c-1}(1-u)^{q-c-1}(1-t u z)^{-a} .
\end{aligned}
$$

The required integrations are all straightforward, yielding

$$
\begin{aligned}
{ }_{3} F_{2}\left(1,1, \frac{5}{2} ; 3,4 ; z\right)= & -\frac{4}{z}\left(1-\frac{4}{z}\right)\left[\ln \left(\frac{z}{4}\right)+2 \tanh ^{-1} \sqrt{1-z}\right] \\
& +\frac{2}{z}+\frac{16}{z^{3}}-\frac{8}{z^{2}}\left(1+\frac{2}{z}\right) \sqrt{1-z}
\end{aligned}
$$

Inserting (D18) into (D16) at last gives us

$$
C(\boldsymbol{\Delta})=\frac{9}{16 \pi a^{2}}\left[\left(2+X^{2}\right) \sqrt{1-X^{2}}-X^{2}\left(4-X^{2}\right) \tanh ^{-1} \sqrt{1-X^{2}}\right],
$$

which is true for $X \leq 1$.

\section{Computation of $\mathcal{L}\left(\boldsymbol{x}-\boldsymbol{x}^{\prime}\right)$}

We now turn to the evaluation of $\mathcal{L}\left(\boldsymbol{x}-\boldsymbol{x}^{\prime}\right)$. This function may be determined by the same procedure used to determine $C(\boldsymbol{\Delta})$. Since Eq. (3.10) expresses $\mathcal{L}\left(\boldsymbol{x}-\boldsymbol{x}^{\prime}\right)$ as the

convolution of $\mathcal{D}$ with a logarithm, we may use (3.11) and (D10) to immediately write 


$$
\widetilde{\mathcal{L}}(\boldsymbol{q})=\frac{1}{2 q^{2}}\left\{1-\frac{9}{(a q)^{6}}[\sin (a q)-(a q) \cos (a q)]^{2}\right\} .
$$

Once again we encounter an expression with a fake pole at the origin: the $\boldsymbol{q} \rightarrow 0$ limit is actually

$$
\lim _{q \rightarrow 0} \tilde{\mathcal{L}}(\boldsymbol{q})=\frac{a^{2}}{10}
$$

So, to invert the Fourier transform, we may employ the same trick as was used on Eq. (D13): after the angular integration we replace the Bessel function of order zero by one of order $2 \epsilon$ and integrate term-by-term. After verifying that all of the poles in $\epsilon$ do indeed cancel, we are left with

$$
\begin{aligned}
\mathcal{L}\left(\boldsymbol{x}-\boldsymbol{x}^{\prime}\right)=\frac{1}{64 \pi} & {\left[-\left(1-X^{2}\right)\left(8+8 X^{2}-X^{4}\right) \ln \left(\frac{X^{2}}{4}\right)\right.} \\
& -\frac{1}{6}\left(1-X^{2}\right)\left(169-47 X^{2}+7 X^{4}\right) \\
+ & \left.\frac{1}{6}-\frac{9 X^{8}}{64}{ }_{4} F_{3}\left(1,1, \frac{5}{2}, 4 ; 3,5,5 ; X^{2}\right)\right] .
\end{aligned}
$$

This expression is valid only for $X \leq 1$. Eq. (D22) displays the precise form predicted for $\mathcal{L}\left(\boldsymbol{x}-\boldsymbol{x}^{\prime}\right)$ at the end of Appendix B: it is a power series in $X^{2}$ plus $\ln X^{2}$ times another series in $X^{2}$. No odd powers of $X$ appear in this series since we employed a rotationally invariant correlation function depending upon the vector $\boldsymbol{x}-\boldsymbol{x}^{\prime}$, as opposed to an arbitrary function of $\left|\boldsymbol{x}-\boldsymbol{x}^{\prime}\right|$.

The hypergeometric function may be eliminated from (D22) by combining (D17) with Eq. (7.512.12) of Ref. 32]:

$$
\begin{aligned}
{ }_{4} F_{3}(a, b, c, d ; p, q, r ; z)= & \frac{\Gamma(p) \Gamma(q) \Gamma(r)}{\Gamma(b) \Gamma(p-b) \Gamma(c) \Gamma(q-c) \Gamma(d) \Gamma(r-d)} \\
& \times \int_{0}^{1} d s \int_{0}^{1} d t \int_{0}^{1} d u s^{b-1}(1-s)^{p-b-1} t^{c-1}(1-t)^{q-c-1} \\
& \quad \times u^{d-1}(1-u)^{r-d-1}(1-s t u z)^{-a}
\end{aligned}
$$

from which we learn that

$$
\begin{aligned}
{ }_{4} F_{3}\left(1,1, \frac{5}{2}, 4 ; 3,5,5 ; z\right)= & -\frac{64}{9 z}\left(1-\frac{9}{z}+\frac{8}{z^{3}}\right)\left[\ln \left(\frac{z}{4}\right)+2 \tanh ^{-1} \sqrt{1-z}\right] \\
& -\frac{128}{9 z^{2}}\left(1+\frac{13}{z}-\frac{14}{z^{2}}\right) \sqrt{1-z} \\
& +\frac{224}{27 z}-\frac{64}{z^{2}}+\frac{256}{z^{3}}-\frac{1792}{9 z^{4}} .
\end{aligned}
$$

The result of inserting (D24) into (D22) is precisely (5.12) except for the step-function. For $X>1$, it is easy to combine the integral representation for $\mathcal{L}$ given in Eq. (3.10) with the integral form of $C$ from Eq. (5.9) to show that that $\mathcal{L}\left(\boldsymbol{x}-\boldsymbol{x}^{\prime}\right)$ vanishes in this region. 


\section{Computation of $L\left(\boldsymbol{x}-\boldsymbol{x}^{\prime}\right)$}

Finally, we come to $L\left(\boldsymbol{x}-\boldsymbol{x}^{\prime}\right)$. We should like to begin with the Fourier transform function $\tilde{L}(\boldsymbol{q})$. Note that (2.25) implies

$$
\boldsymbol{q}^{2} \tilde{L}(\boldsymbol{q})=2 \widetilde{\mathcal{L}}(\boldsymbol{q}) .
$$

Unfortunately, this relation is not sufficient to determine $\tilde{L}(\boldsymbol{q})$ uniquely: we may add an arbitrary multiple of $\delta^{2}\left(\boldsymbol{q}^{2}\right)$ to $\tilde{L}(\boldsymbol{q})$ without contradicting Eq. (D25). According to the integral representation of $L\left(\boldsymbol{x}-\boldsymbol{x}^{\prime}\right), L(\mathbf{0})$ vanishes. Therefore, we could adjust this arbitrary term so that this is true when we invert the Fourier transform. Operationally, we may simply invert the expression implied by (D25) using the same method we have used on the other integrals in this Appendix and drop the pole and constant terms. Although this procedure lacks the satisfying feeling that comes when one sees the poles cancel automatically, it does produce the correct result, as has been verified by a direct numerical evaluation of the integral in (3.9). The bottom line is that for $X \leq 1$ we find

$$
\begin{aligned}
L\left(\boldsymbol{x}-\boldsymbol{x}^{\prime}\right)=\frac{a^{2}}{4 \pi} & {\left[\left(X^{2}-\frac{X^{6}}{8}+\frac{X^{8}}{128}\right) \ln \left(\frac{X^{2}}{4}\right)\right.} \\
& +\frac{3 X^{2}}{2}-\frac{9 X^{4}}{8}+\frac{5 X^{6}}{24}-\frac{5 X^{8}}{384} \\
& \left.+\frac{9 X^{10}}{12800}{ }_{4} F_{3}\left(1,1, \frac{5}{2}, 4 ; 3,6,6 ; X^{2}\right)\right]
\end{aligned}
$$

whereas for $X \geq 1$

$$
L\left(\boldsymbol{x}-\boldsymbol{x}^{\prime}\right)=-\frac{a^{2}}{20 \pi}\left[\frac{93}{20}+\ln \left(\frac{X^{2}}{4}\right)\right] .
$$

This latter result may be obtained inserting the integral representation (5.9) of $C$ into the integral representation (3.9) of $L$. Using the identity (D23) to evaluate the hypergeometric function appearing in (D26) gives:

$$
\begin{aligned}
{ }_{4} F_{3}\left(1,1, \frac{5}{2}, 4 ; 3,6,6 ; z\right)= & -\frac{20}{9 z}\left(5-\frac{80}{z}+\frac{640}{z^{3}}+\frac{128}{z^{4}}\right)\left[\ln \left(\frac{z}{4}\right)+2 \tanh ^{-1} \sqrt{1-z}\right] \\
& -\frac{8}{9 z^{2}}\left(25+\frac{982}{z}-\frac{2984}{z^{2}}-\frac{1488}{z^{3}}\right) \sqrt{1-z} \\
& +\frac{500}{27 z}-\frac{8000}{27 z^{2}}+\frac{1600}{z^{3}}-\frac{6400}{3 z^{4}}-\frac{3968}{3 z^{5}}
\end{aligned}
$$

Eqs. (D26)-(D28) combine to reproduce the expression presented in Eq. (5.11). 


\section{REFERENCES}

* Electronic address: lam@physics.mcgill.ca

$\dagger \quad$ Electronic address: mahlon@physics.mcgill.ca

[1] L. McLerran and R. Venugopalan, Phys. Rev. D49, 2233 (1994). hep-ph/9309289

[2] L. McLerran and R. Venugopalan, Phys. Rev. D49, 3352 (1994). hep-ph/9311205

[3] L. McLerran and R. Venugopalan, Phys. Rev. D50, 2225 (1994). hep-ph/9402335

[4] A. Ayala, J. Jalilian-Marian, L. McLerran, and R. Venugopalan, Phys. Rev. D52, 2935 (1995). hep-ph/9501324

[5] A. Mueller, Nucl. Phys. B307, 34 (1988).

[6] A. Mueller, Nucl. Phys. B317, 573 (1989).

[7] A. Mueller, Nucl. Phys. B335, 115 (1990).

[8] A. Mueller, Eur. Phys. J. A1, 19 (1998). hhep-ph/9710531

[9] Yu. Kovchegov and A. Mueller, Nucl. Phys. B529, 451 (1998). hep-ph/9802440

[10] A.H. Mueller, "Parton saturation at small $x$ and in large nuclei," hep-ph/9904404.

[11] A. Ayala, J. Jalilian-Marian, L. McLerran, and R. Venugopalan, Phys. Rev. D53, 458 (1996). hep-ph/9508302

[12] J. Jalilian-Marian, A. Kovner, L. McLerran, and H. Weigert, Phys. Rev. D55, 5414 (1997). hep-ph/9606337

[13] J. Jalilian-Marian, A. Kovner, A. Leonidov, and H. Weigert, Nucl. Phys. B504, 415 (1997). hep-ph/9701284

[14] J. Jalilian-Marian, A. Kovner, A. Leonidov and H. Weigert, Phys. Rev. D59, 014014 (1999). hep-ph/9706377

[15] J. Jalilian-Marian, A. Kovner and H. Weigert, Phys. Rev. D59, 014015 (1999). hepph/9709432

[16] J. Jalilian-Marian, A. Kovner, A. Leonidov and H. Weigert, Phys. Rev. D59, 034007 (1999); erratum Phys. Rev. D59, 099903 (1999). hep-ph/9807462

[17] J. Jalilian-Marian and X.-N. Wang, "Small $x$ gluons in nuclei and hadrons," hep$\mathrm{ph} / 9902411$.

[18] A. Kovner and J. Guilherme Milhano, "Vector potential versus color charge density in low- $x$ evolution," hep-ph/9904420.

[19] L. McLerran and R. Venugopalan, Phys. Lett. B424, 15 (1998). nucl-th/9705055

[20] M. Gyulassy and L. McLerran, Phys. Rev. C56, 2219 (1997). nucl-th/9704034]

[21] S.V. Molodtsov, A.M. Snigirev and G.M. Zinovjev, Phys. Lett. B443, 387 (1998). hep$\mathrm{ph} / 9804418$

[22] L.V. Gribov, E.M. Levin, and M.G. Ryskin, Phys. Rep. 100, 1 (1983).

[23] L. McLerran, "Three lectures on the physics of small $x$ and high gluon density," hepph/9903536.

[24] R. Venugopalan, "Parton saturation, production, and equilibrium in high energy nuclear collisions," hep-ph/9907209.

[25] L.N. Lipatov, Sov. J. Nucl. Phys. 20, 95 (1975); V.N. Gribov and L.N. Lipatov, Sov. J. Nucl. Phys. 15, 438 (1972); Yu. Dokshitzer, Sov. Phys. JETP 46, 641 (1977).

[26] G. Alterelli and G. Parisi, Nucl. Phys. B126, 298 (1977).

[27] Yu. Kovchegov, Phys. Rev. D54, 5463 (1996). hhep-ph/9605446

[28] G. Curci, W. Furmanski, R. Petronzio, Nucl. Phys. B175, 27 (1980). 
[29] J.C. Collins and D.E. Soper, Nucl. Phys. B194, 445 (1982).

[30] S.J. Brodsky and G.P. Lepage in Perturbative Quantum Chromodynamics, edited by A.H. Mueller (World Scientific, 1989).

[31] A.H. Mueller in Frontiers in Particle Physics, Cargese 1994, edited by M. Levy, J. Iliopoulos, R. Gastmans, and J.-M. Gerard, NATO Advanced Study Institute Series B, Physics, Vol. 350, (Plenum Press, 1995).

[32] I.S. Gradshteyn and I.M. Ryzhik, Table of Integrals, Series, and Products (Academic Press, Inc., 1980).

[33] See for example, G. Baym, Lectures on Quantum Mechanics (The Benjamin/Cummings Publishing Company, 1969), p. 429. 


\section{FIGURES}

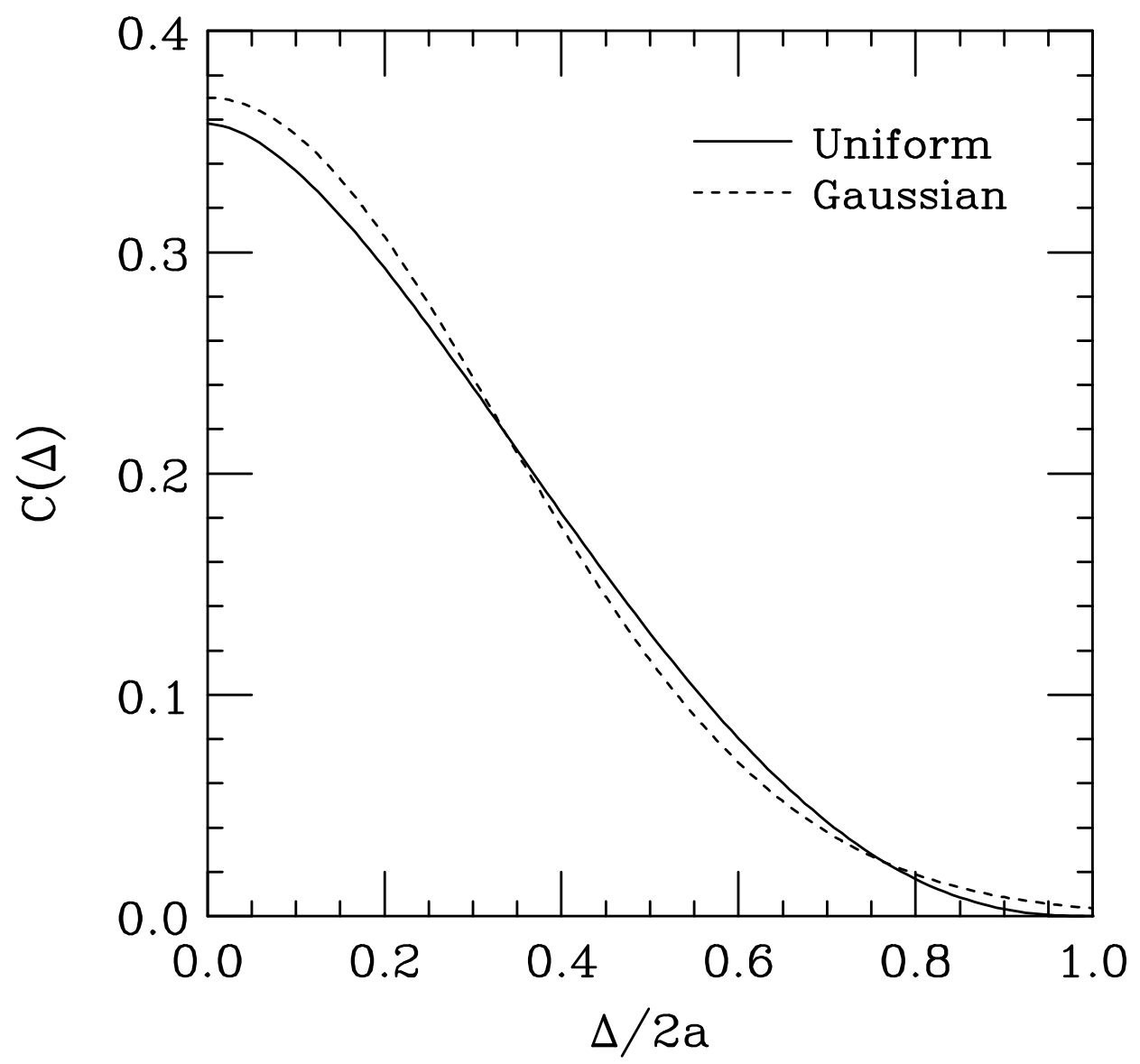

FIG. 1. The smooth part of the two-point charge density correlation function in Kovchegov's model [27, as given in Eqs. (5.9) (uniform quark/nucleon distribution) and (5.10) (Gaussian quark/nucleon distribution). The nucleon size parameters $a$ have been chosen according to Eq. (5.20) so that resulting gluon number densities match in the ultraviolet limit. In the uni-

form case, $C(\Delta)$ vanishes for $\Delta>2 a$. In the Gaussian case, there is a Gaussian tail in this region. 


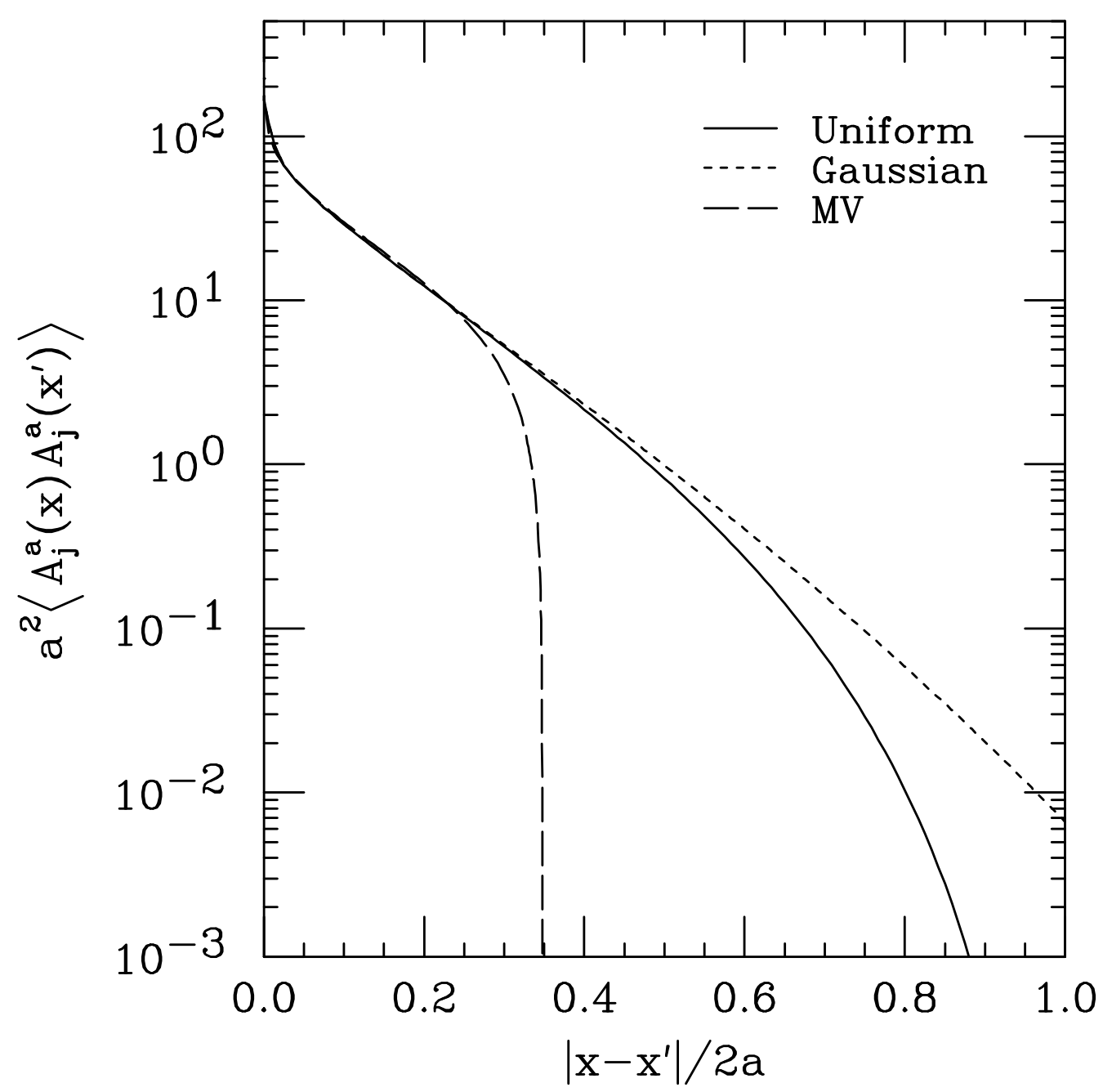

FIG. 2. The trace of the two-point vector potential correlation function in position space. The Fourier transform of this function is proportional to the gluon number density [c.f. Eq. (2.2)]. The nucleon size parameters $a$ and $\Lambda_{\mathrm{QCD}}$ have been chosen according to Eqs. (5.20) and (5.21) so that these functions match in the ultraviolet $\left(\left|\boldsymbol{x}-\boldsymbol{x}^{\prime}\right| \rightarrow 0\right)$ limit. The longitudinal coordinates $\left(x^{-}, x^{\prime-}\right)$ have been fixed at a place where $a^{2} \chi=20$. Plotted are the results of Ref. [12] (labelled "MV") as well as results using Kovchegov's model [27] with a uniform or Gaussian distribution of quarks and nucleons. 


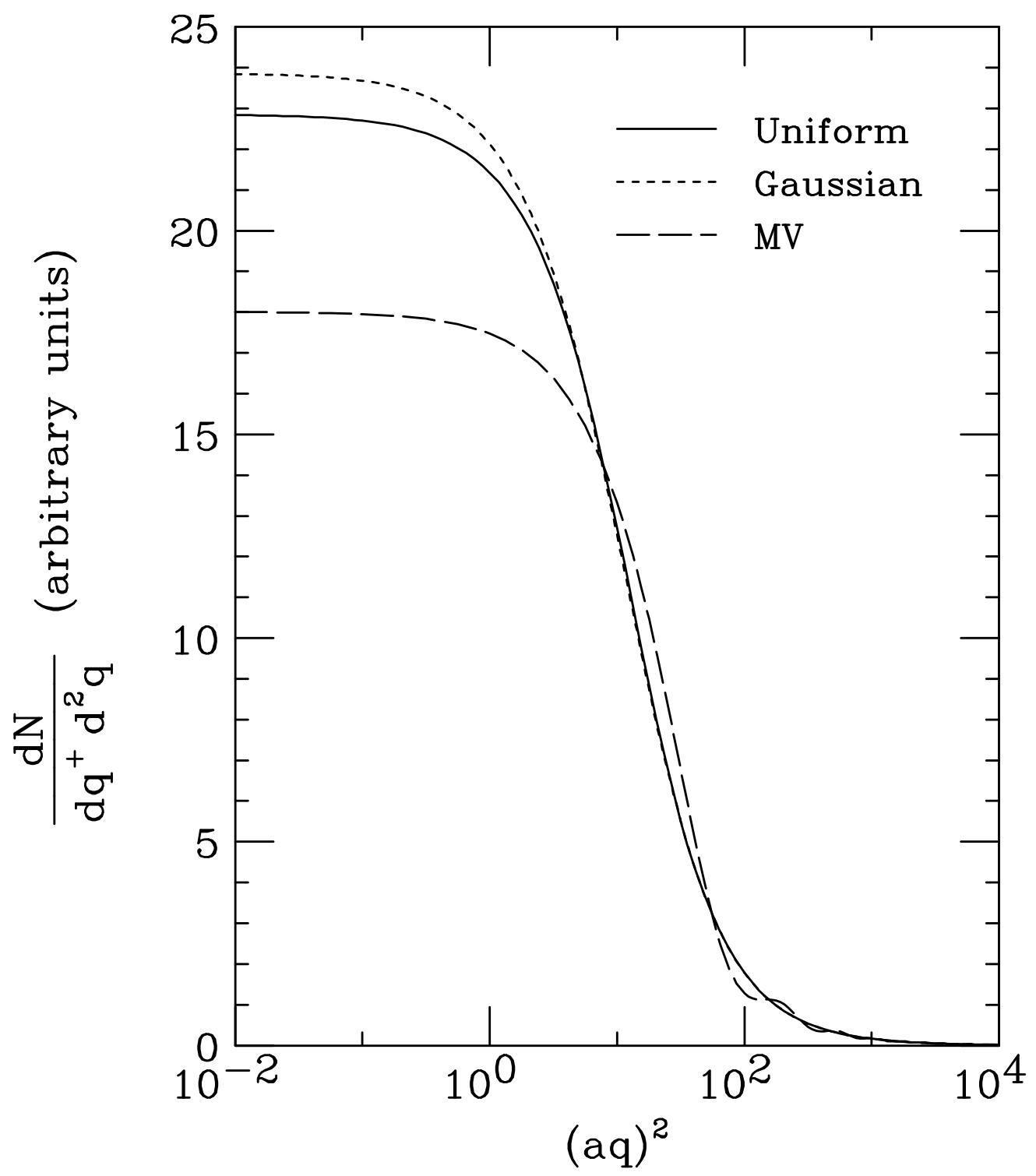

FIG. 3. Plot of the gluon number density (4.9) at fixed $q^{+}$. The nucleon size parameters $a$ and $\Lambda_{\mathrm{QCD}}$ have been chosen according to Eqs. (5.20) and (5.21) so that these functions match in the ultraviolet limit. The total charge squared per unit area has been set to $a^{2} \mathcal{X}_{\infty}=20$. Plotted are the results of Ref. [12] (labelled "MV") as well as results using Kovchegov's model [27] with a uniform or Gaussian distribution of quarks and nucleons. 


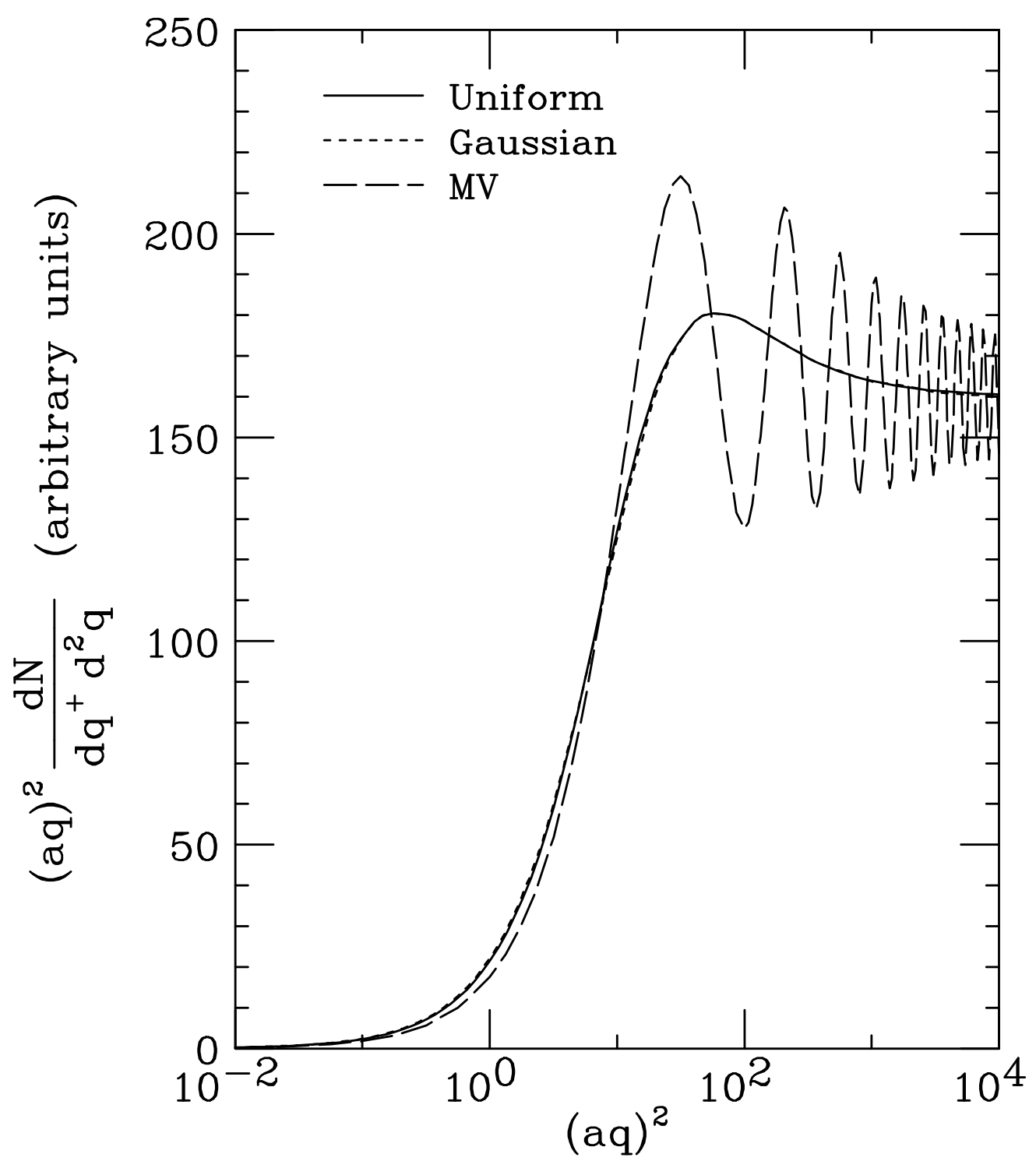

FIG. 4. Plot of $(a q)^{2}$ times the gluon number density (4.9) at fixed $q^{+}$. This combination has been chosen to enhance the high momentum part of the distribution and show the approach to the predicted $1 / \boldsymbol{q}^{2}$ ultraviolet behavior. The nucleon size parameters $a$ and $\Lambda_{\mathrm{QCD}}$ have been chosen according to Eqs. (5.20) and (5.21) so that these functions match in this limit. The total charge squared per unit area has been set to $a^{2} \mathcal{X}_{\infty}=20$. Plotted are the results of Ref. [12] (labelled "MV") as well as results using Kovchegov's model [27] with a uniform or Gaussian distribution of quarks and nucleons. 


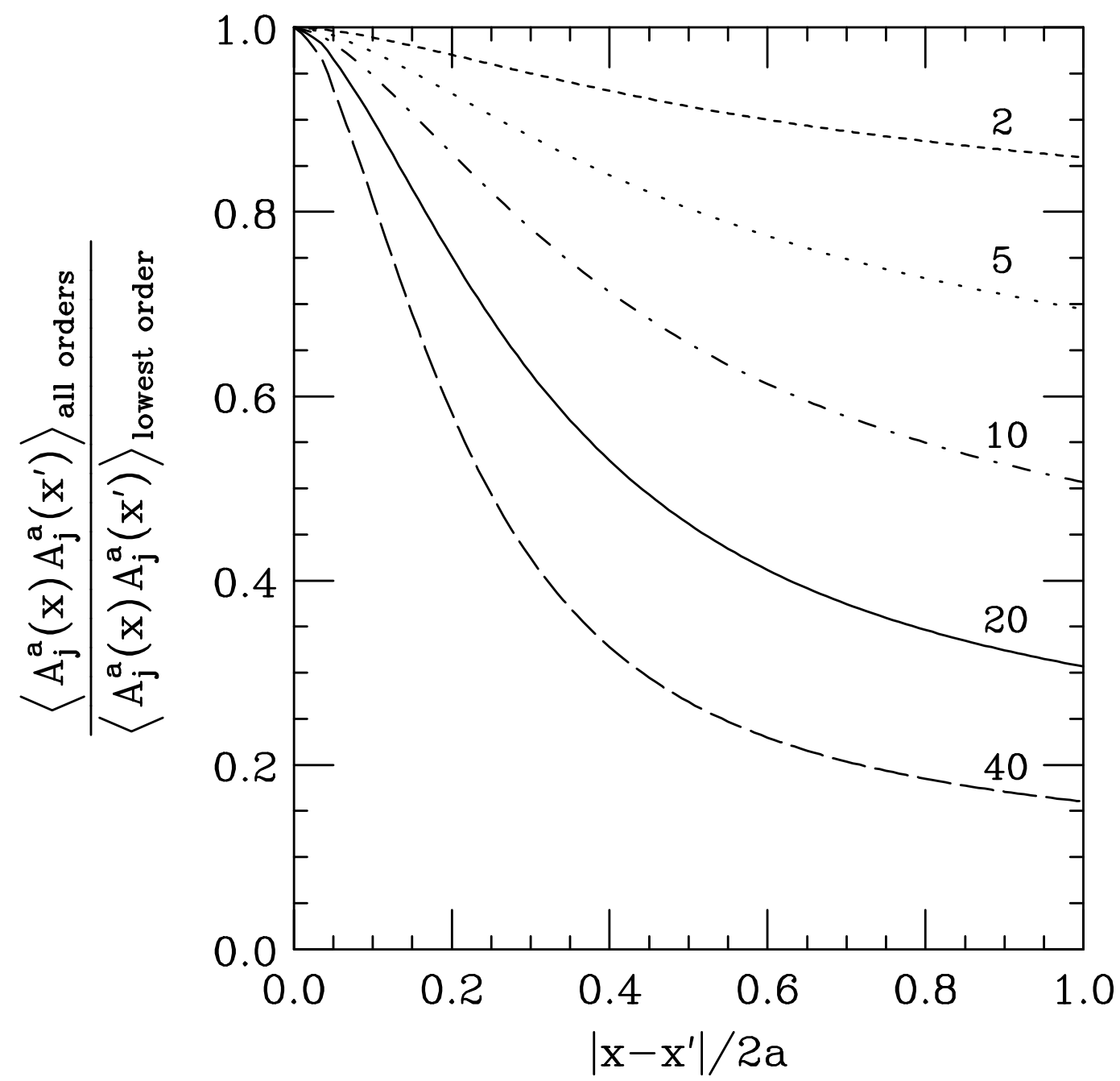

FIG. 5. The effect of the non-Abelian terms on the trace of the correlation function. Plotted is the ratio of the all-orders result to the lowest-order (purely Abelian) result using the uniform version of Kovchegov's model [27. The different curves are for fixed values of the longitudinal coordinates $\left(x^{-}, x^{-}\right)$such that $a^{2} \chi=2,5,10,20$, and 40 . 


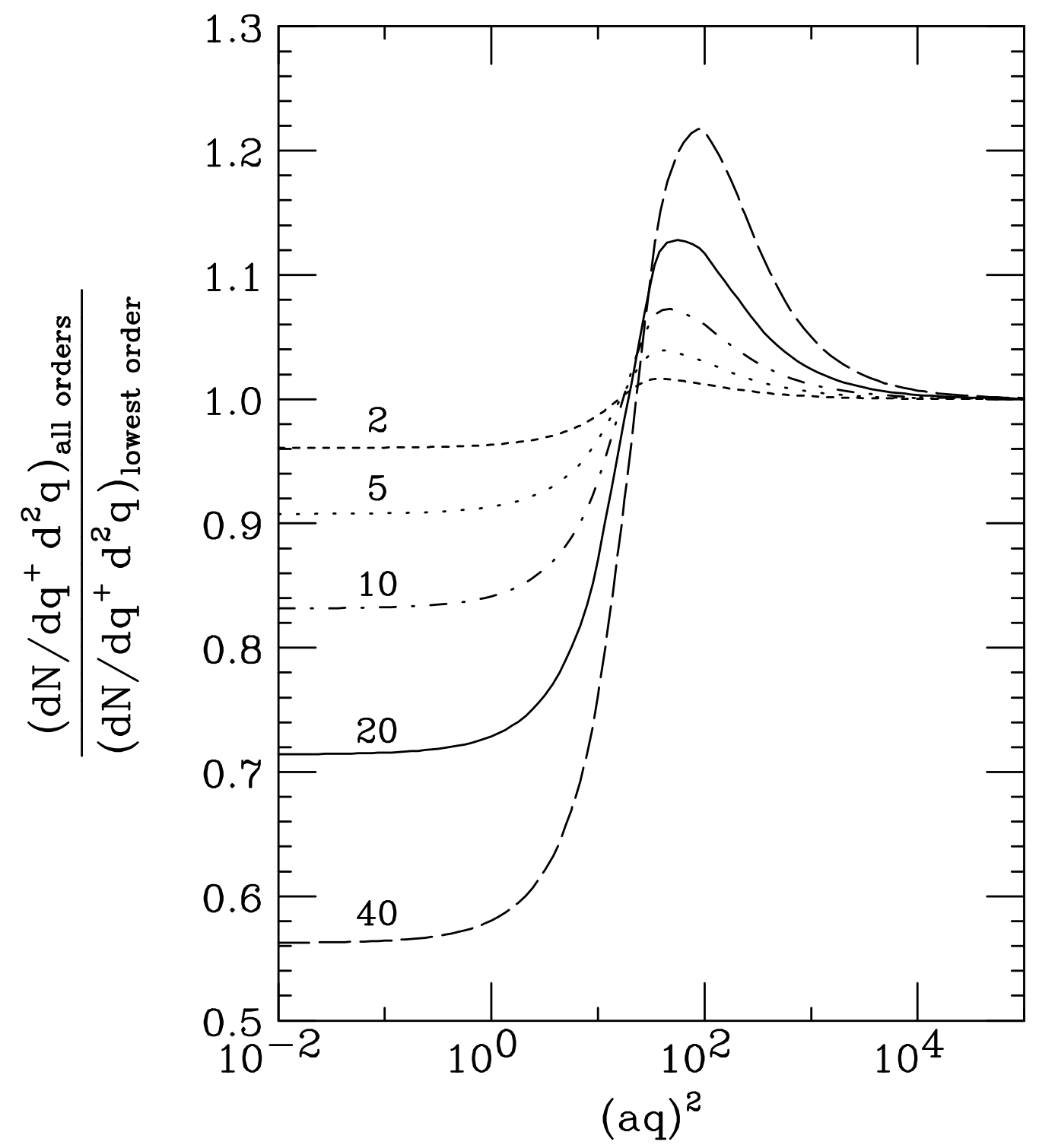

FIG. 6. The effect of the non-Abelian terms in momentum space. Plotted is the ratio of the all-orders gluon number density to the lowest-order (purely Abelian) gluon number density at fixed $q^{+}$using the uniform version of Kovchegov's model [27]. The different curves are for $a^{2} \mathcal{X}_{\infty}=2,5$, 10,20 , and 40 . 

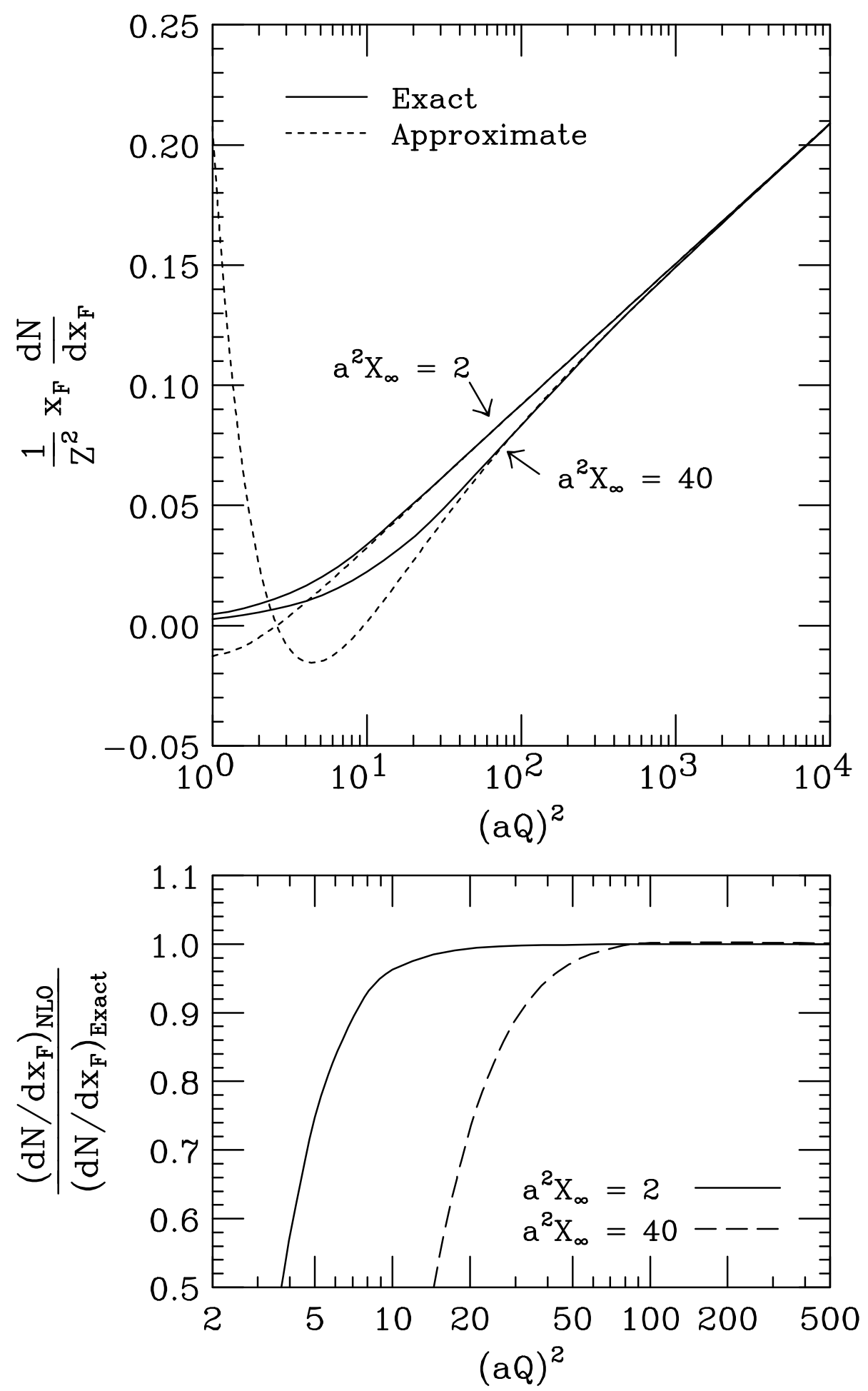

FIG. 7. Top: Gluon structure function $x_{F} g\left(x_{F}, Q^{2}\right)=x_{F} d N / d x_{F}$ divided by the total color charge-squared for a large nucleus. Plotted is a comparison of the next-to-leading order approximation (4.33) with the exact result obtained by numerical integration of (4.22) for two different values of $\mathcal{X}_{\infty}$. Bottom: The ratio of the next-to-leading order approximation to the exact result in the region where the approximation begins to break down. 

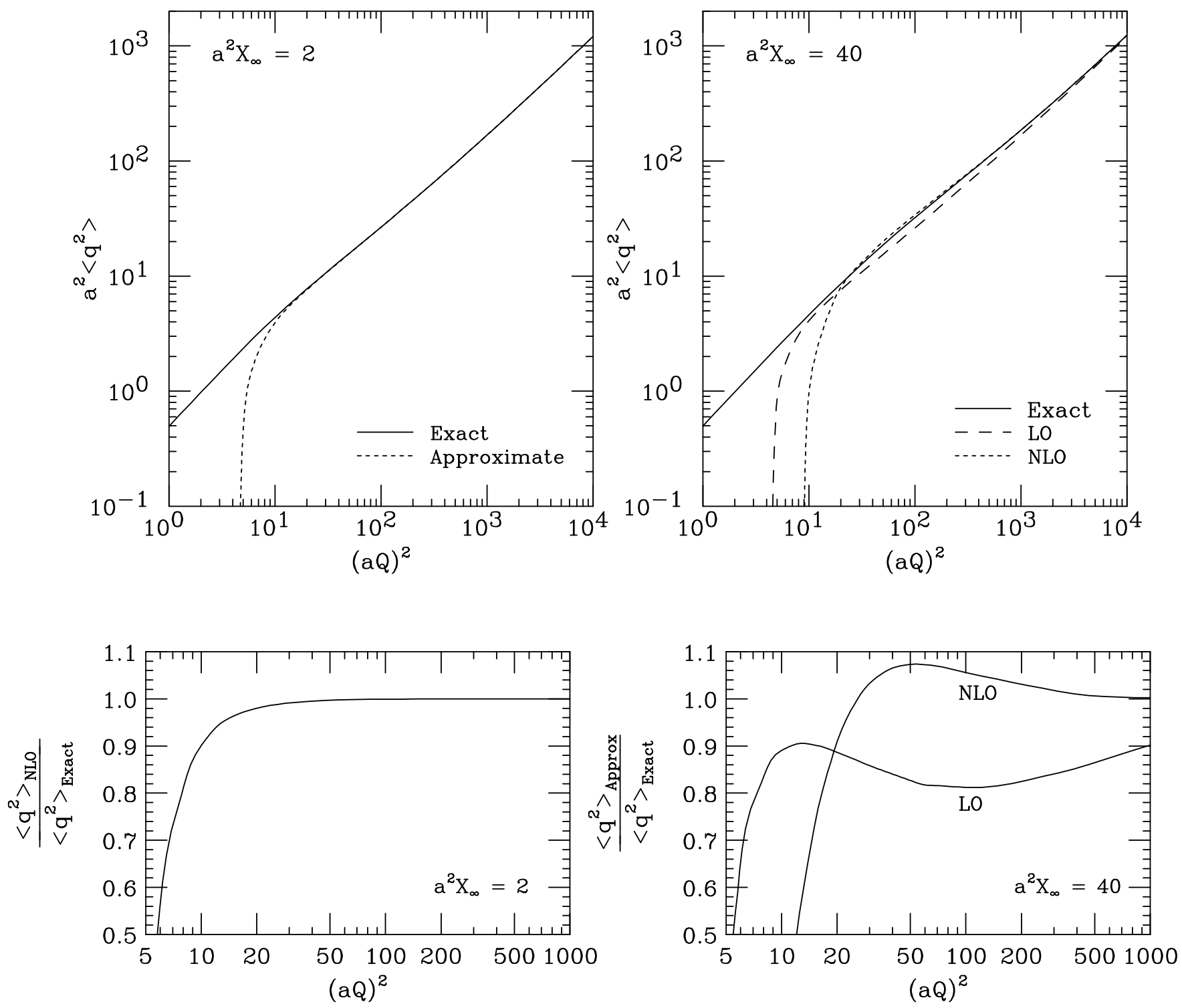

FIG. 8. Top: Mean transverse momentum-squared as a function of the parton resolution scale $Q^{2}$. Plotted is a comparison of the next-to-leading order approximation (4.36) with the exact result obtained by numerical integration for $a^{2} \mathcal{X}_{\infty}=2$, and a comparison of the leading order, next-to-leading order, and exact results for $a^{2} \mathcal{X}_{\infty}=40$. Bottom: The ratio of the approximate results to the exact results in the region where the approximation begins to break down. 


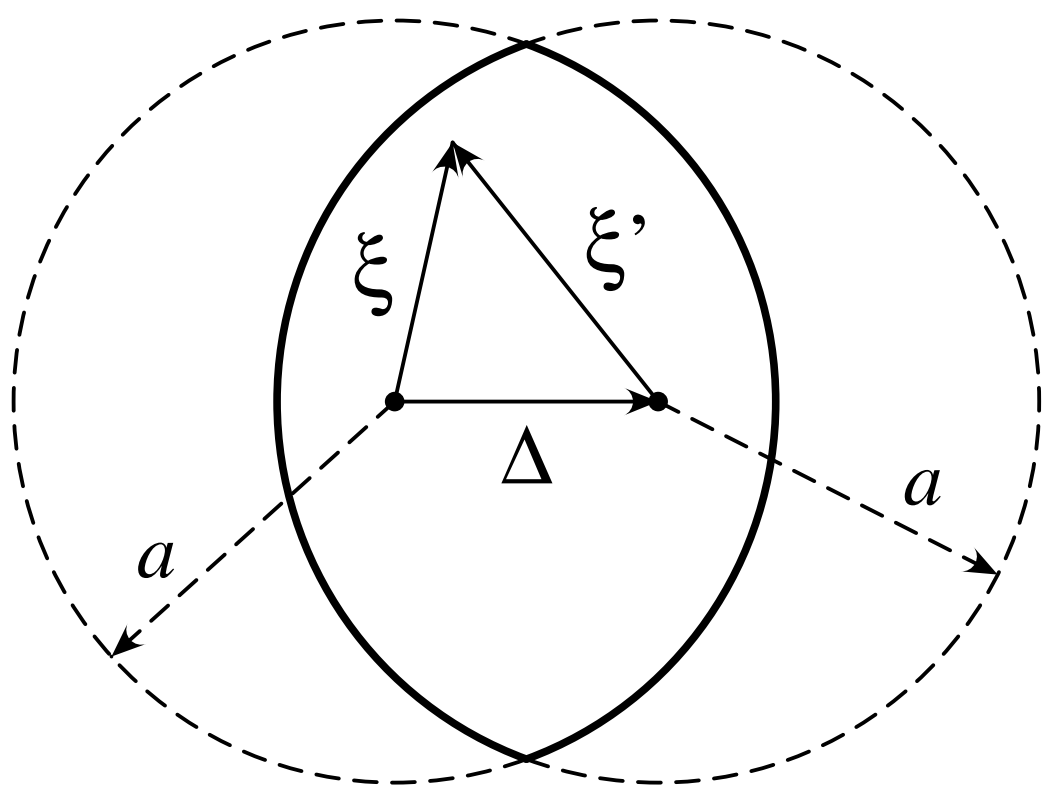

FIG. 9. The integration region encountered in Eq. (D5) for the smooth part of the correlation function in the uniform version of Kovchegov's model [27]. 